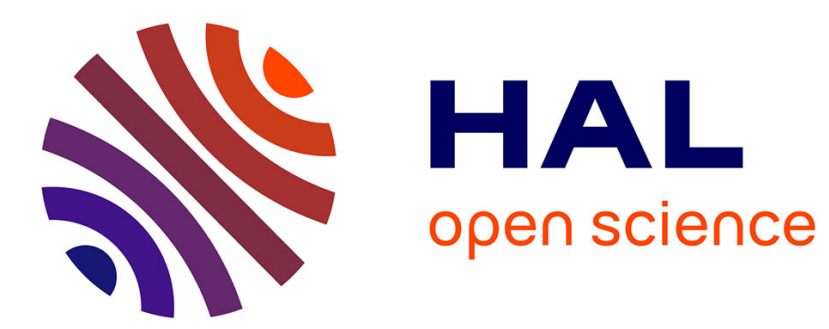

\title{
Acetylene, 12C2H2: new CRDS data and global vibration-rotation analysis up to $8600 \mathrm{~cm}-1$
}

Michel Herman

\section{To cite this version:}

Michel Herman. Acetylene, 12C2H2: new CRDS data and global vibration-rotation analysis up to 8600 cm-1. Molecular Physics, 2008, 106 (21-23), pp.2581-2605. 10.1080/00268970802620709 . hal00513240

\author{
HAL Id: hal-00513240 \\ https://hal.science/hal-00513240
}

Submitted on 1 Sep 2010

HAL is a multi-disciplinary open access archive for the deposit and dissemination of scientific research documents, whether they are published or not. The documents may come from teaching and research institutions in France or abroad, or from public or private research centers.
L'archive ouverte pluridisciplinaire HAL, est destinée au dépôt et à la diffusion de documents scientifiques de niveau recherche, publiés ou non, émanant des établissements d'enseignement et de recherche français ou étrangers, des laboratoires publics ou privés. 


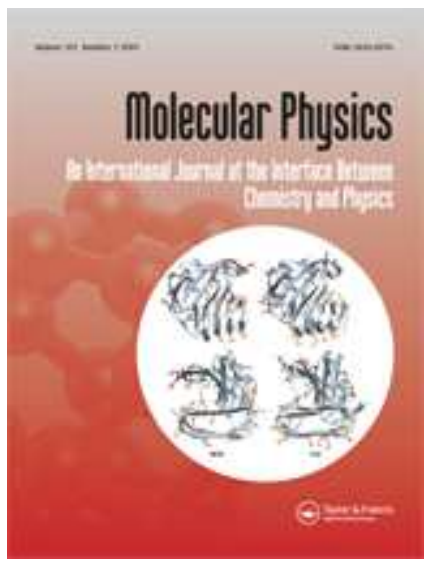

\section{Acetylene, 12C2H2: new CRDS data and global vibration- rotation analysis up to $8600 \mathrm{~cm}-1$}

\begin{tabular}{|r|l|}
\hline Journal: & Molecular Physics \\
\hline Manuscript ID: & TMPH-2008-0267.R1 \\
\hline Manuscript Type: & Full Paper \\
\hline Date Submitted by the \\
Author: & 04-Nov-2008 \\
\hline Complete List of Authors: & $\begin{array}{l}\text { Herman, Michel; Université Libre de Bruxelles, Chimie quantique et } \\
\text { Photophysique }\end{array}$ \\
\hline Keywords: & acetylene, CRDS, global fit, IR spectra \\
\hline
\end{tabular}

\section{s scholarONE" \\ Manuscript Central}




\title{
Acetylene, ${ }^{12} \mathrm{C}_{2} \mathrm{H}_{2}$ : new CRDS data and global vibration- rotation analysis up to $8600 \mathrm{~cm}^{-1}$ \\ S. Robert ${ }^{1 *}$, M. Herman ${ }^{1}$
}

A. Fayt $^{2}$

A. Campargue ${ }^{3}$, S. Kassi ${ }^{3}$, A. Liu ${ }^{3}$, L. Wang ${ }^{3}$

G. Di Lonardo ${ }^{4}$, L. Fusina ${ }^{4}$

\author{
${ }^{1}$ Service de Chimie quantique et Photophysique CP160/09 \\ Faculté des Sciences \\ Université Libre de Bruxelles (U.L.B.) \\ Av. Roosevelt, 50 \\ B-1050, Bruxelles \\ Belgium \\ ${ }^{2}$ Laboratoire de Spectroscopie Moléculaire \\ Université Catholique de Louvain \\ Chemin du Cyclotron, 2 \\ B-1348 Louvain-La-Neuve \\ Belgium \\ ${ }^{3}$ Laboratoire de Spectrométrie Physique \\ Université Joseph Fourier de Grenoble Laboratoire de Spectrométrie \\ Physique (associated with CNRS, UMR 5588), Université Joseph Fourier de \\ Grenoble, B.P. 87, 38402 Saint-Martin-d'Hères Cedex, France \\ ${ }^{4}$ Dipartimento di Chimica Fisica e Inorganica \\ Università di Bologna \\ Viale Risorgimento, 4 \\ I-40136, Bologna \\ Italy \\ * ARC / FRIA researcher \\ Pages: 57 \\ Figures: 5 \\ Tables: 8 \\ Send mail to Prof. M. Herman Email mherman@ulb.ac.be
}




\begin{abstract}
The absorption spectrum of ${ }^{12} \mathrm{C}_{2} \mathrm{H}_{2}$ has been recorded using cavity ringdown spectroscopy and analyzed in the ranges $6000-6356 \mathrm{~cm}^{-1}$ and $6667-7015$ $\mathrm{cm}^{-1}$. Fourteen new bands have been identified and additional $J$-lines were assigned in 10 already known bands. These new data, together with the published vibration-rotation absorption lines of ${ }^{12} \mathrm{C}_{2} \mathrm{H}_{2}$ accessing vibrational states up to $8600 \mathrm{~cm}^{-1}$ have been gathered in a database. The resulting set includes 12137 transitions involving 186 different $k=l_{4}+l_{5}$ sub-states, with $l_{\text {i }}$ the angular momentum quantum number associated to the $i$ degenerate bending vibration. These lines have been fitted simultaneously to spectroscopic parameters through $J$-dependent Hamiltonian matrices exploiting the vibrational polyad or cluster block-diagonalization, in terms of the pseudo quantum numbers $N_{r}=5 v_{1}+3 v_{2}+5 v_{3^{+}} v_{4^{+}} v_{5}$ and $N_{s}=v_{1}+v_{2}+v_{3}$, also accounting for $k$ and $e / f$ parities and $u / g$ symmetry. Modes 1 and 2 correspond to the symmetric $\mathrm{CH}$ and $\mathrm{CC}$ stretchings, mode 3 to the antisymmetric $\mathrm{CH}$ stretch, and modes 4 and 5 to the trans- and cisdegenerate bendings, respectively. The fit was successfully achieved, with a dimensionless standard deviation of 0.92 , leading to the determination of 266 effective vibration-rotation parameters.
\end{abstract}




\section{Introduction}

The high resolution spectrum of acetylene, ${ }^{12} \mathrm{C}_{2} \mathrm{H}_{2}$, has been already investigated in the $1.5 \mu \mathrm{m}$ spectral range using Fourier transform infrared spectroscopy (FTIR) ${ }^{1-4}$. The strong $v_{1}+v_{3}$ band, of metrological relevance, is observed in this range, together with various combination and hot bands. Nowadays, cavity ringdown spectroscopy (CRDS) (e.g. ${ }^{5,6}$ ) allows the experimental sensitivity to be significantly increased with respect to FTIR measurements. We have used CW-CRDS ${ }^{7,8}$ to record the acetylene spectrum around $1.5 \mu \mathrm{m}$, in Grenoble. Experimental details are provided in section 2 and the results of the band-by-band analyses performed in Bologna, between $6000-6356 \mathrm{~cm}^{-1}$ and $6667-7015 \mathrm{~cm}^{-1}$, are listed in section 3 .

The new spectra revealed very weak bands compared to the existing literature data. Their analysis was facilitated by unpublished global constants obtained in Brussels from the simultaneous fit of many sets of already known vibration-rotation lines. Such a procedure, which was already achieved for ${ }^{13} \mathrm{CH}^{12} \mathrm{CH}$ up to $6750 \mathrm{~cm}^{-1}$ using a set of programs developed in Louvain ${ }^{9}$, exploits the very regular vibrational energy pattern in acetylene, as recently reviewed in ${ }^{10}$. The present investigation stimulated an update of the still unpublished results of our ${ }^{12} \mathrm{C}_{2} \mathrm{H}_{2}$ global fit, to include the new CRDS data reported here. The data base gathering the published vibration-rotation lines in ${ }^{12} \mathrm{C}_{2} \mathrm{H}_{2}$ involving vibrational states up to $8600 \mathrm{~cm}^{-1}$, is summarized in 
section 4 . This range was selected to include all states observed through cold and hot bands in the present CRDS spectra. The global model is presented in section 5 . The results, also updating a previously published global analysis by Perevalov et al. ${ }^{11}$, are detailed in section 6 and discussed in section 7 , before concluding in section 8 .

The conventional normal mode numbering in acetylene is used throughout this paper, with 1 to 5 corresponding to the symmetric $\mathrm{CH} \sigma_{\mathrm{g}}^{+}\left(v_{1}\right)$ and $\mathrm{CC} \sigma_{\mathrm{g}}^{+}\left(v_{2}\right)$ stretchings, the antisymmetric $\mathrm{CH}$ stretch $\sigma_{\mathrm{u}}^{+}\left(v_{3}\right)$, and the trans $-\pi_{\mathrm{g}}\left(v_{4}\right)$ and cis- $\pi_{\mathrm{u}}\left(v_{5}\right)$ degenerate bendings. The latter are characterized by the usual bending angular momentum quantum numbers, $l_{4}$ and $l_{5}$, with $k$ $=l_{4}+l_{5}$.

\section{Experimental details}

The CW-CRDS spectra of acetylene were recorded with the same spectrometer used in studies of species of atmospheric relevance such as $\mathrm{H}_{2} \mathrm{O}$ ${ }^{12}$ and ${ }^{12} \mathrm{CO}_{2}{ }^{13-15}$ or $\mathrm{O}_{3}{ }^{16}$. The fibered $\mathrm{CW}-\mathrm{CRDS}$ spectrometer and the procedure adopted for an accurate calibration of the wavenumber scale of the recorded spectra were described in Refs. ${ }^{12}{ }^{14}$. Fig. 1 shows an overview of the acetylene absorption spectrum recorded by FTS at ULB, highlighting the 
two spectral sections presently investigated. The $6000-6356 \mathrm{~cm}^{-1}$ and $6667-7015 \mathrm{~cm}^{-1}$ regions were selected for the present CRDS recordings as they correspond to transparency windows located between strong absorption bands. These two regions were covered by using a set of 18 and 12 Distributed Feed-Back (DFB) diode lasers, respectively. Only the range $6122-6130 \mathrm{~cm}^{-1}$ could not be recorded due to the lack of diode.

[ Insert figure 1 about here ]

The tuning range of each DFB laser is about $7 \mathrm{~nm}\left(\sim 30 \mathrm{~cm}^{-1}\right)$ by temperature variation from -10 to $65^{\circ} \mathrm{C}$. The typical sensitivity (noise equivalent absorption $\alpha_{\min } \sim 2-5 \times 10^{-10} \mathrm{~cm}^{-1}$ ), wide spectral coverage and the four to five decades linear dynamic range, make it an ideal tool for high sensitivity absorption spectroscopy in the important atmospheric window of transparency around $1.5 \mu \mathrm{m}$. The CRDS cell fitted by the super mirrors is $1.40 \mathrm{~m}$ long. The typical ringdown times were of the order of $60 \mu \mathrm{s}$. A few hundred ringdown events were averaged for each spectral data point, taking some $220 \mathrm{~ms}$ per averaged point, and the duration of a temperature scan was about 70 minutes for each DFB laser. The pressure, measured by a capacitance gauge, as well as the ringdown cell temperature, were monitored during the spectrum recording. Different series of recordings were performed 
for pressure values of 667,1333 and $2666 \mathrm{~Pa}$ depending on the spectral region. The DFB line width is much smaller than the Doppler width and the observed resolution is mostly Doppler limited in the adopted pressure conditions. In cases of overlapped lines, whenever it was possible, a deconvolution by means of Voigt profile was performed, keeping the full width at half maximum at a value observed for close ${ }^{12} \mathrm{C}_{2} \mathrm{H}_{2}$ isolated lines.

The wavenumber calibration of the spectrum is based on the wavelength values measured by a wavemeter (Burleigh WA1640) during the DFB frequency scan. When necessary, it was further refined by simply stretching the frequency scale (with an origin at 0 ) in agreement with highly accurate positions of reference lines due to impurities. $\mathrm{H}_{2}{ }^{16} \mathrm{O}$ line positions as provided by the HITRAN database ${ }^{18}$ were used as reference lines. The obtained absolute wavenumber calibration is estimated accurate within better than $\pm 2 \times 10^{-3} \mathrm{~cm}^{-1}$.

The achieved sensitivity and dynamics are illustrated in Fig. 2 on a small section of the spectrum near $6235 \mathrm{~cm}^{-1}$. It is significantly improved compared to the previous investigation by Keppler et al. ${ }^{3}$, based on FTIR and up to $352.5 \mathrm{~m}$ long absorption path. The improvement is best demonstrated by the analysis of 14 additional, new bands in the present spectra. 
[ Insert figure 2 about here ]

The spectrum analysis is complicated by the presence of absorption lines due to several impurities: $\mathrm{H}_{2} \mathrm{O}, \mathrm{CH}_{4}$ and $\mathrm{C}_{2} \mathrm{H}_{4}$. Methane transitions, as reported in the HITRAN database ${ }^{18}$, were observed in the region of the strong $2 v_{3}$ band near $6000 \mathrm{~cm}^{-1}$, while $\mathrm{C}_{2} \mathrm{H}_{4}$ lines belonging to the $v_{5}+v_{9}$ band at $6151 \mathrm{~cm}^{-1}$ were identified between 6095 and $6210 \mathrm{~cm}^{-1}{ }^{19}$. However, the main problem was the presence of acetone, which is added to stabilize acetylene when it is compressed. On the overview FTIR spectrum of Fig. 1, the broad absorption features of acetone are clearly observed below 6050 $\mathrm{cm}^{-1}$. They induced additional losses which strongly decreased the ringdown times and therefore the sensitivity near $6000 \mathrm{~cm}^{-1}$. In this range, the noise equivalent absorption increased up to ${ }_{\min } \sim 3 \times 10^{-9} \mathrm{~cm}^{-1}$, to be compared to $2-5 \times 10^{-10} \mathrm{~cm}^{-1}$, typically.

\section{Description of the spectra and band-by-band analyses}

Fourteen new vibrational bands have been identified in the CRDS spectra, arising from the ground state or from excited bending states with $v_{4^{+}} v_{5} \leq 2$. They altogether involve 23 different upper vibrational states. These bands are gathered in Table 1 together with 10 bands already reported by Keppler 
et al. ${ }^{3}$ whose rotational assignments have been extended. Band centres and vibrational term values are also reported in Table 1 . The states are identified using the vibrational quantum numbers in acetylene, defined in the introduction. The assignments correspond, as usual, to the dominant zero order at the lowest existing $J$-value for the $k$ sub-state in the corresponding polyad as resulting from the global fit.

In the $6000-6356 \mathrm{~cm}^{-1}$ range, the most prominent features are the $R(J)$ lines of the $2 v_{1}\left(\Sigma_{\mathrm{g}}^{+}\right) \leftarrow v_{5}\left(\Pi_{\mathrm{u}}\right)$ hot band extending from 6000 to $6050 \mathrm{~cm}^{-1}$ (see Fig.1). At $6011 \mathrm{~cm}^{-1}$ the red degraded $Q$ branch of the $v_{1}+v_{2}+v_{4}+v_{5}\left(\Sigma_{\mathrm{u}}^{+}\right) \leftarrow v_{4}$ $\left(\Pi_{\mathrm{g}}\right)$ is observed together with the corresponding $R$ branch up to $6068 \mathrm{~cm}^{-1}$. Other weaker $Q$ branches centred at 6030,6052 and $6093.5 \mathrm{~cm}^{-1}$ are present, corresponding to the $v_{1}+v_{2}+2 v_{5}\left(\Sigma_{\mathrm{g}}^{+}\right) \leftarrow v_{5}\left(\Pi_{\mathrm{u}}\right), v_{1}+v_{2}+v_{5}\left(\Pi_{\mathrm{u}}\right) \leftarrow$ G.S. and $2 v_{2}+3 v_{5}$ $\left(\Pi_{\mathrm{u}}\right) \leftarrow$ G.S. newly observed bands. The corresponding $P$ and $R$ transitions have also been identified, with the exception of the $P(J)$ lines of the $6052 \mathrm{~cm}^{-1}$ band, despite their position can be precisely calculated. This intensity perturbation, probably due to a high order Coriolis type vibration-rotation interaction, is not fully understood. Fig. 3 illustrates the very weak $Q$ branch of the $v_{1}+v_{2}+2 v_{5}\left(\Sigma_{\mathrm{g}}^{+}\right) \leftarrow v_{5}\left(\Pi_{\mathrm{u}}\right)$ band superimposed with the $R$ branch of the $2 v_{1}\left(\Sigma_{\mathrm{g}}^{+}\right) \leftarrow v_{5}\left(\Pi_{\mathrm{u}}\right)$ band, while the very weak $Q$ branch of the $2 v_{2}+3 v_{5}\left(\Pi_{\mathrm{u}}\right)$ $\leftarrow$ G.S. band is presented in Fig. 4. A parallel band centred at $6079.7 \mathrm{~cm}^{-1}$ 
has been assigned to $v_{1}+v_{4}+3 v_{5}\left(\Sigma_{u}^{+}\right) \leftarrow$ G.S.. Between 6095 and $6210 \mathrm{~cm}^{-1}$ the spectrum is dominated by the strong lines of the $\mathrm{C}_{2} \mathrm{H}_{4}$ impurity, which overlap the weaker $\mathrm{C}_{2} \mathrm{H}_{2}$ transitions, making the assignment very difficult. In fact ${ }_{2}$ only a few transitions have been added to previously observed bands ${ }^{3}$.

\section{[ Insert figures 3 and 4 about here ]}

At both ends of the region between 6667 and $7015 \mathrm{~cm}^{-1}$ the wings of the strong $v_{1}+v_{3}$ and $v_{1}+v_{3}+v_{4}$ band systems, centred at 6556 and $7141 \mathrm{~cm}^{-1}$, respectively, are visible. In the FTIR spectrum only the $2 v_{1}+v_{5}\left(\Pi_{u}\right) \leftarrow v_{4}\left(\Pi_{g}\right)$ and $v_{2}+v_{3}+2 v_{5}\left(\Sigma_{\mathrm{u}}^{+}\right) \leftarrow$ G.S. assigned in Keppler et al. ${ }^{3}$ at 6804.7 and $6690 \mathrm{~cm}^{-1}$, respectively, have been identified. Most of the bands observed in the CRDS spectrum are of parallel type, $\Sigma_{\mathrm{g} / \mathrm{u}}^{+} \leftarrow \Sigma_{\mathrm{u} / \mathrm{g}}^{+}, \Pi_{\mathrm{g} / \mathrm{u}} \leftarrow \Pi_{\mathrm{u} / \mathrm{g}}$ and $\Delta_{\mathrm{g} / \mathrm{u}} \leftarrow \Delta_{\mathrm{u} / \mathrm{g}}$. In a few $\Pi_{\mathrm{g} / \mathrm{u}} \leftarrow \Pi_{\mathrm{u} / \mathrm{g}}$ bands also the very weak $Q$ branches have been observed and assigned. In the higher wavenumber side two perpendicular bands, $\Delta_{\mathrm{g}} \leftarrow \Pi_{\mathrm{u}}$ and $\Sigma_{\mathrm{g}}^{+} \leftarrow \Pi_{\mathrm{u}}$, have been identified, both centered at about $6956 \mathrm{~cm}^{-1}$ and leading to states of the same manifold.

The spectral analysis was made difficult by the superposition of the different bands of ${ }^{12} \mathrm{C}_{2} \mathrm{H}_{2}$ with lines due to impurities (in particular $\mathrm{H}_{2} \mathrm{O}$ and $\left.\mid \mathrm{C}_{2} \mathrm{H}_{4}\right)$ or to the ${ }^{13} \mathrm{CH}^{12} \mathrm{CH}$ isotopologue. In fact ${ }_{2}$ lines without intensity 
alternation due to transitions already reported ${ }^{6}$ in twelve bands for this molecule have been observed mainly in the $6667-7015 \mathrm{~cm}^{-1}$ range.

The rotational analysis of the bands listed in Table 1 was performed using standard procedures. The energy of the rovibrational levels of the $\Sigma^{+}, \Pi$, and $\Delta$ states was calculated according to the expression:

$$
\begin{aligned}
& T(v, J)=G_{c}+B_{v} J(J+1)-D_{v}[J(J+1)]^{2}+H_{v}[J(J+1)]^{3} \\
& \pm 1 / 2\left\{q_{v}[J(J+1)]+q_{v}^{J}[J(J+1)]^{2}+q_{v}^{J J}[J(J+1)]^{3}\right\}
\end{aligned}
$$

with $q_{v}, q_{v}^{J}, q_{v}^{J J}=0$ for $l=0, \Sigma$ states, and the centre term value defined as

$$
G_{c}=G_{v}-B_{v} k^{2}-D_{v} k^{4}
$$

and $G_{v}$ the vibrational term value. In Eq. (1) upper signs refer to $e$-symmetry states, lower signs to $f$-symmetry states.

The constants resulting from the band-by-band analysis are listed in

Table 1 . The term values of the lower states of the transitions were calculated from literature data ${ }^{20}$. The number of lines assigned in the branches and the number of fitted lines are also indicated for each band. 
[ Insert Table 1 about here ]

Transitions whose wavenumbers differed from the corresponding calculated values by more than a chosen limit, ranging from 0.001 to 0.003 $\mathrm{cm}^{-1}$, were excluded from the final cycle of the least-squares procedure. Most of these excluded line positions correspond to high $J$ values which cannot be reproduced by using Eq. (1), in which the number of fitted constants is limited to the sextic coefficient $H^{\prime}$. Actually, for many bands, additional coefficients in the $J(J+1)$ expansion could as well be fitted and statistically determined. This behaviour is typical whenever resonances occur, which are not accounted for in the band-by-band rotational model of Eq. (1). For the Deleted: same reason, some of the distortion constants listed in Table 1 are anomalously large or negative. One can notice that the same upper state $v_{1}+v_{2}+v_{4}+v_{5}\left(\Sigma_{u}^{+}\right)$is involved in both cold and hot bands. Upper state rotational constants slightly differ for these bands reaching the same upper state, because of the different number and measurement precision of the rotational lines included in the fitting procedure.

Additional, weaker lines remain to be identified in the range presently studied. Some can tentatively be assigned to impurities in the sample, others probably arise from the studied species. In fact ${ }_{2}$ a few sequences of lines with the appropriate intensity alternation have been observed which cannot be assigned for several reasons. They are probably $R$ (or $P$ ) branches whose 
corresponding $P$ (or $R$ ) counterpart is outside the investigated spectral region. With the help of the predicted line positions by means of the parameters of the global fit three $R$ branches of this kind have been assigned to the $\Pi \leftarrow \Pi$ bands centred at 6662,6672 and $6674 \mathrm{~cm}^{-1}$ (see Table 1). Unassigned series could also correspond to hot bands from states with vibrational excitation higher than those considered in the predictions, limited to $v_{4^{+}} v_{5} \cdot 2$. Another possibility, which has not been exploited, is represented by zero-order forbidden transitions with $|\Delta k|=2$. These additional structures will be considered at a later stage of the global analysis.

All line wavenumbers from the rotational analyses reported in Table 1, together with their assignments, are attached as Supplementary Material. Observed-calculated values obtained from the global procedure described in section 6, rather than from the band-by-band parameters listed in Table 1, are also provided.

\section{Data base}

The data base consists of the assigned line wavenumbers from the literature, within the upper vibrational state energy limit of $8600 \mathrm{~cm}^{-1}$. This limit was selected to include all new states from the present CRDS data. 
The bands involved in the data base are identified in Table 2. All data, except for the Raman ones by Bermejo et al. ${ }^{21,22}$, come from various absorption techniques. It is interesting to notice that, besides the bending range investigated using FTIR ${ }^{20,23,24}$, some low energy bands were only reported in the early literature, investigated using high resolution spectrographs, by Rao and coworkers ${ }^{25-28}$. The quality of these data, although optimal at the time of their publication, may possibly be improved nowadays. Some more recent data recorded using FTIR have, actually since been published but the related list of assigned lines could unfortunately not be made available by the authors ${ }^{29}$.

The data base includes the vibration-rotation wavenumbers from the systematic investigation by Jacquemart and co-workers, using FTIR ${ }^{23,30-37}$. Additional conventional FTIR results around $1.5^{2,3}$ and $1.17 \mu \mathrm{m}{ }^{38}$, as well as lines around $2.5 \mu \mathrm{m}$ reported from very sensitive FTIR experiments using a femto laser as absorption source ${ }^{39}$, are included. Finally, 14 new bands presently observed by CW-CRDS are also part of the data base, together with additional lines in 10 previously reported ones.

[ Insert Table 2 about here ]

Data concerning very highly excited bending states in the range considered were reported by Field and co-workers using resolved laser 
induced fluorescence from the $\tilde{A}$ excited electronic state ${ }^{40}$. They were, however, not considered because of the required extension in the size of the Hamiltonian matrices, further detailed in section 6 .

When two published sets of lines were available for the same band, the information has been merged in various ways, depending on the quality of the measurements. For instance, in several cases FTIR data were selected for the lower $J$-lines and complemented with the higher $J$-line wavenumbers reported from more sensitive laser-based techniques. In few cases, the latter data have been included for some of the lower $J$ values. However, only one line position was selected in cases where several appear in the literature. These finer details are not reported in Table 2. Each data set is then separately treated in the fitting procedure in terms of weighting factors, as further detailed in section 6 .

The data base amounts to 12137 vibration-rotation lines accessing states up to $8600 \mathrm{~cm}^{-1}$. They arise from bands linking 186 different $l$ substates (counting $e$ and $f$ components separately). All states involved in the data base are gathered in Table 3 , together with their centre term value $G_{\mathrm{c}}$, calculated from the global constants resulting from the procedure detailed in the next sections. It is interesting to notice that, because of the $u / g$ selection rule, this data set is significantly reduced compared to ${ }^{13} \mathrm{CH}^{12} \mathrm{CH}$, for which 
about the same number of lines was gathered from the literature, up to the limit of $6750 \mathrm{~cm}^{-1}$ as compared to $8600 \mathrm{~cm}^{-1}$ in the present case.

\section{[ Insert Table 3 about here ]}

It must be emphasized that, as the same states are observed from spectra recorded in different wavelength ranges and using various experimental conditions, spectral calibration is a critical issue when performing global fitting. We satisfactorily checked that the line measurement accuracy, described in the references just mentioned, was comparable to the quality of the standard deviation of the final fit.

\section{Vibration-rotation Hamiltonian}

For several isotopologues of acetylene, the vibrational state energies could be gathered into so-called polyads or vibrational clusters up to high excitation, block-diagonalizing the global vibrational Hamiltonian ${ }^{41-46}$. Each of these blocks within the Hamiltonian matrix image of the molecule (MIME) shows a similar structure, accounting for the same set of anharmonic resonances, all characterized by well-defined interaction matrix elements. As a consequence of this regularity, new constants of the motion, $N_{r}$ and $N_{s}$, can be defined in ${ }^{12} \mathrm{C}_{2} \mathrm{H}_{2}$ and also in ${ }^{13} \mathrm{CH}^{12} \mathrm{CH}$ and ${ }^{13} \mathrm{C}_{2} \mathrm{H}_{2}{ }^{47,48}$ : 


$$
\begin{aligned}
& N_{r}=5 v_{1}+3 v_{2}+5 v_{3}+v_{4}+v_{5} \\
& N_{s}=v_{1}+v_{2}+v_{3}
\end{aligned}
$$

Interesting issues of dynamical and statistical natures support this very specific vibrational MIME structure $\left(\right.$ e.g. $\left.{ }^{40,49-53}\right)$.

Inclusion of rotation in the global picture has been already achieved for ${ }^{12} \mathrm{C}_{2} \mathrm{H}_{2}$ by Perevalov and coworkers ${ }^{11,54,55}$, simultaneously considering 7290 lines involving states up to $9700 \mathrm{~cm}^{-1}$. No detailed related discussion was, however, ever published to complement the limited information provided in a symposium proceeding ${ }^{5}{ }^{54}$. Concerning ${ }^{13} \mathrm{CH}^{12} \mathrm{CH}$, the 12703 vibration-rotation lines reported in the literature up to $6750 \mathrm{~cm}^{-1}$ could be simultaneously fitted and all reproduced within experimental precision $(3 \sigma)$, by Fayt and coworkers ${ }^{9}$. Since the vibrational structure in ${ }^{12} \mathrm{C}_{2} \mathrm{H}_{2}$ is very close to the one in ${ }^{13} \mathrm{CH}^{12} \mathrm{CH}$, apart for restrictive $u / g$ selection rules, the vibrational energy patterns and MIME are almost identical for both species and the same strategy and programs adopted for ${ }^{13} \mathrm{CH}^{12} \mathrm{CH}^{9,46}$ were applied here to ${ }^{12} \mathrm{C}_{2} \mathrm{H}_{2}$.

Deleted:

The vibrational interactions active in ${ }^{12} \mathrm{C}_{2} \mathrm{H}_{2}$ are known to be $3 / 245$, $14 / 35,15 / 34,1 / 244,1 / 255,44 / 55$ and $11 / 33^{43}$. The notation $i / j k k(i i / j j)$ refers to anharmonic resonance interaction terms of the type 
$\Delta v_{i}= \pm 1, \Delta v_{j}=\mp 1, \Delta v_{k}=\mp 2 \quad\left(\Delta v_{i}= \pm 2, \Delta v_{j}=\mp 2\right) \quad$ characterized by the interaction constant $K_{i / j k k}\left(K_{i i / j j}\right)$. The related main coupling terms fulfill the $\Delta k=0$ selection rule in various possible ways, thus with either $\Delta l_{4}=\Delta l_{5}=0$, or $\Delta l_{4}=-\Delta l_{5} \neq 0$. Higher order terms, labeled $O_{i j / m n}$, apply for $\Delta k= \pm 2$ coupling schemes. For completeness, vibrational and rotational interaction terms in the global Hamiltonian are defined in Table 4. Only some of the anharmonic resonance terms are explicitly provided in this Table, namely those relevant to the $3 / 245$ and $44 / 55$ anharmonic couplings in eqs. (13-16) and (17-19), respectively. The selection of higher order terms was adapted to the results of the global fit presented in the next section.

[ Insert Table 4 about here ]

As detailed in ${ }^{9}$, vibration-rotation polyads include states with different values of $k=l_{4}+l_{5}$, either even or odd. The $e$ and $f$ partition defined in ${ }^{56}$ is also included, with the symmetrized basis as defined in ${ }^{57}$ for pentatomic molecules. Also, $g$ and $u$ symmetry is used to classify the total wavefunction and to further block-diagonalize the Hamiltonian. Finally, the resulting vibration-rotation matrices are diagonal in $J$. Each block is characterized by the pseudo-quantum numbers $N_{r}$ and $N_{s}$ defined in Eqs (3) and (4), the total angular momentum quantum number $J$, and the symmetry properties $e / f$ and $u / g$. Each block is therefore labeled using the $\left\{N_{r}, N_{s}, J, e / f, u / g\right\}$ notation. 


\section{Fitting procedure and results}

The package of computer programs developed in Louvain-La-Neuve and described in ${ }^{57,58}$ was used for the fitting procedure. The matrices were set to include states up to $v_{b}=7(b \cdot 4$ or 5$)$ and $k_{\max }=7$, with the constraint $v_{4}+v_{5} \leq 8$. In this way, the most relevant data were accounted for without significantly increasing computer time. This limit, however ${ }_{2}$ prevented data concerning highly excited bending states ${ }^{40}$ to be considered, as mentioned in section 4. Polyads were nevertheless built to include levels up to $16000 \mathrm{~cm}^{-1}$, to allow for later developments.

We used a weighted fitting procedure (weights inversely proportional to the square of the experimental uncertainties) initially accounting for the estimated accuracy $(3 \sigma)$ mentioned by the authors, which is mainly dependent on the used technique and spectral range investigated. For each set of data, the uncertainty was later reduced in order to agree with the corresponding mean (obs.- calc.) value from the global fit, whenever this value was better than the stated experimental accuracy. 
The final selection of parameters, although not expected to be unique, results from a careful fitting strategy. Higher order parameters were added one at a time, depending on both the nature of the specific problem to be solved and on the size and precision of the corresponding lower order parameters. In the end, the number of parameters was systematically reduced removing those statistically undetermined, taking care of not degrading the standard deviation of the fit.

The final global fit included 10750 lines out of 12137 assigned ones. It produced 266 parameters, listed in Table 5. The dimensionless standard deviation of the fit is 0.92 this is defined as:

$$
\hat{\sigma}=\sqrt{\frac{\sum_{i}\left[\left(\tilde{v}_{i}^{\text {obs. }}-\tilde{v}_{i}^{\text {calc. }}\right) / \varepsilon_{i}\right]^{2}}{N-n}}
$$

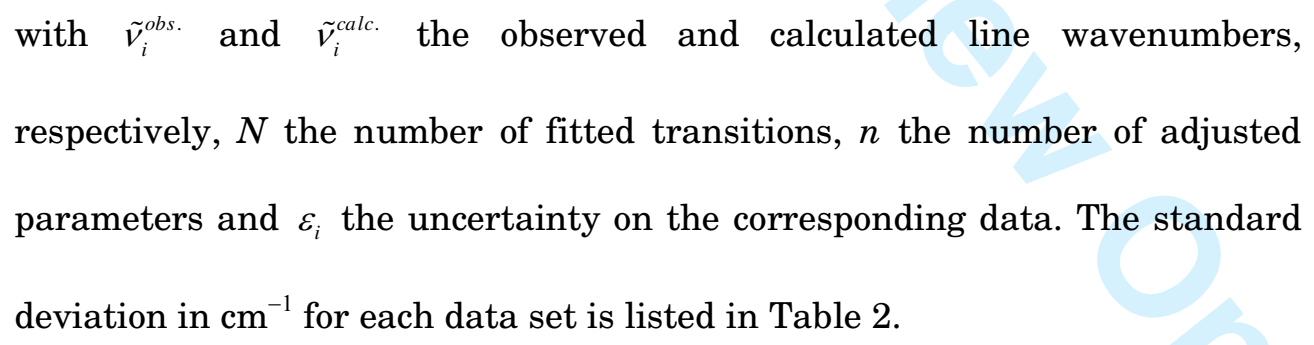
respectively, $N$ the number of fitted transitions, $n$ the number of adjusted parameters and $\varepsilon_{i}$ the uncertainty on the corresponding data. The standard deviation in $\mathrm{cm}^{-1}$ for each data set is listed in Table 2.

[ Insert Table 5 about here ] 
A statistical survey of the quality of the vibration-rotation data of ${ }^{12} \mathrm{C}_{2} \mathrm{H}_{2}$ accounted for in the global fit is presented in Table 6 .

A zero weight has been assigned to 1387 lines for which the fit was not satisfactory, meaning all those with observed-calculated value larger than three times the uncertainty $\varepsilon_{i}$. Since we do not have access to all spectra, we could not systematically check the quality of these rejected lines. About 80\% of these correspond to very low or high $J$-values. They are thus most likely weaker or overlapped lines in different bands, less precisely measured. We could clearly assess this in ${ }^{13} \mathrm{CH}^{12} \mathrm{CH}{ }^{9}$, as further discussed below. Their rejection, only occurs because they are characterized by the same uncertainties as for the other more accurately measured lines in the same bands and does therefore not affect the global picture.

We identified specific high order Coriolis type vibration-rotation interaction schemes, 14/22 in particular, likely to explain the rejection of the remaining 20\%. This coupling sometimes affects many rotational levels within the same vibrational sub-state. It is e.g. the case for $\left(v_{1} v_{2} v_{3} v_{4} v_{5}, l_{4} l_{5}\right)=$ $(10012,10) \Pi^{f}$ sub-state at $5381.56 \mathrm{~cm}^{-1}$, perturbed by the $(02002,02) \Delta^{f}$ one. Significant changes in the programs would be required to account for these additional $\Delta k=1$ couplings, affecting only a very small fraction, about $2 \%$, of the information available in the present data base. Depending on the various 
accounted for in acetylene, the block diagonal structure of the Hamiltonian might even be destroyed, leading to an infinite matrix representation.

The total fraction of the data base which was rejected (11.4\%) is actually of the same order of magnitude as in ${ }^{13} \mathrm{CH}^{12} \mathrm{CH}(7.6 \%){ }^{9}$. The slight increase might result from more pronounced high order Coriolis type vibration-rotation effects. The better coherence of the data set in ${ }^{13} \mathrm{CH}^{12} \mathrm{CH}$, because a larger fraction of them results from a single series of high quality FTIR spectra, probably also contributes to this comparison. One should furthermore account for the energy ranges considered in both cases, up to $8600 \mathrm{~cm}^{-1}$ in ${ }^{12} \mathrm{C}_{2} \mathrm{H}_{2}$ and $6750 \mathrm{~cm}^{-1}$, only, in ${ }^{13} \mathrm{CH}^{12} \mathrm{CH}$. More complex interaction schemes due to a denser pattern of states at the higher energies considered are thus to be unraveled in the symmetric isotopologue. In addition, since the number of data is almost identical in both data bases, the information content is significantly higher in the asymmetric isotopologue, imposing more efficient constraints on the fitting procedure.

[ Insert Table 6 about here ]

\section{Global constants}


The large number of fitted data (10750) ensures the overall statistical meaning of the procedure, despite the large number of determined parameters (266). However, severe correlation problems can not be avoided when performing global vibration-rotation fits and certainly characterize several of the present effective parameters. The use of constraints in the fitting procedure as explained in ${ }^{57}$ significantly contributed to the final selection of higher order terms in the model. These terms for ${ }^{12} \mathrm{C}_{2} \mathrm{H}_{2}$ are different from those determined in the similar fit of ${ }^{13} \mathrm{CH}^{12} \mathrm{CH}{ }^{9}$. It is interesting to notice that the number of parameters is higher in ${ }^{12} \mathrm{C}_{2} \mathrm{H}_{2}(266)$, compared to ${ }^{13} \mathrm{CH}^{12} \mathrm{CH}$ (216), due to the inclusion of higher energy vibrational states in the main isotopologue. This involves polyads with larger pseudoquantum numbers and requires higher order dependences in the equations. Nevertheless, the resulting sets of parameters show very good consistency, as demonstrated by the selection listed in Tables 7 and 8. All higher order parameters present very similar values, a trend probably to be expected given the very similar vibrational and rotational properties of these isotopologues.

\section{[ Insert Table 7 about here ]}

Further comparison of the present results on ${ }^{12} \mathrm{C}_{2} \mathrm{H}_{2}$, with those in ${ }^{54}$, is more difficult. Indeed, despite the fact that the basic theoretical models are 
identical, the fitting was neither achieved using the same data base nor adopting the same selection of diagonal and off diagonal interaction terms or the same procedure and constraints. We thus only mention that all leading and higher order rotational terms compare well, but not the vibrational ones. Actually their constants (absolute values in $\mathrm{cm}^{-1}$ ) $x_{11}$ (18.96), $x_{33}(30.02), x_{13}$ (105.29), and $K_{11 / 33}(14.06){ }^{54}$ do not fit the so-called $x-K$ relations, i.e.

$$
x_{11}=x_{33}=1 / 4 x_{13}=1 / 4 K_{11 / 33}
$$

predicted to apply in all symmetric isotopologues of acetylene ${ }^{59}$. These relations are reasonably well fulfilled by the present values, listed in the first column of Table 8.

\section{[ Insert Table 8 about here ]}

It is interesting to notice that the values of the vibrational parameters for ${ }^{12} \mathrm{C}_{2} \mathrm{H}_{2}$ derived from a fit of the vibrational energies only ${ }^{43}$ are very similar to those presently obtained considering vibration-rotation data. This previous set, listed in the second column of Table 8, was derived from vibrational data covering a much broader spectral range, up to the visible region. This agreement gives some feeling about the effective character of the parameters. The same comment applies to the parameters of ${ }^{13} \mathrm{CH}^{12} \mathrm{CH}$, also reported in 
Table 8, from ${ }^{9,46}$. Values for the same parameters in ${ }^{13} \mathrm{C}_{2} \mathrm{H}_{2}{ }^{44}$ and ${ }^{12} \mathrm{C}_{2} \mathrm{D}_{2}{ }^{42}$, resulting from a vibrational fit, are also listed for completeness.

Another test of the reliability of the present set of parameters comes from their predictive efficiency. As already mentioned, the global approach significantly facilitated the assignment of the new weaker bands on the CWCRDS spectrum discussed in section 3. Also, as demonstrated in a preliminary report ${ }^{60}$, the global parameters are presently being used with success to assign new emission spectra from very hot acetylene.

[ Insert figure 5 about here ]

As another example, Fig. 5 details the vibration-rotation states around $7135 \mathrm{~cm}^{-1}$ from data reported in ${ }^{3,38}$, with their reduced energy plotted as function of $J(J+1)$. Only $u$-symmetry vibrational states existing in the range appear on Fig. 5, with those relevant assigned. We have selected the zeroorder state with the largest weight in the eigenvector at the lowest $J$-value to label these eigenstates, as everywhere else in the text. The state of interest, $(10110,10) \Pi^{e, f}$, appears to be perturbed for $J$-values from 21 to 23 for the $f$ symmetry, and from 18 to 21 and 28-29 for the $e$-symmetry. As shown in Fig. 5, level crossings can be identified from the predictions. Predicted crossings agree within no more than a fraction of a wavenumber from the observed 
perturbations, allowing them to be unraveled. The coupling schemes are very complicated. Indeed, the $(10110,10) \Pi^{e, f}$ zero order state is actually strongly coupled to many other zero order states in the $\left\{N_{r}, N_{s}, u\right\}=\{11,2, u\}$ polyad, all with odd $k$ values, such as (11021), through $K_{3 / 245}$, and (01112), through $K_{1 / 255}$. The resulting eigenstate at $7141.5 \mathrm{~cm}^{-1}$ thus shares these various zero-order assignments in the eigenvector composition. On the other hand, several zero order states in the $\{11,1, u\}$ polyad, now all with even $k$ values and involving states such as (01053), (10051) and (00142) also happen to be highly coupled by anharmonic and $\ell$-type resonances. High order Coriolis type vibrationrotation coupling schemes involving e.g. the $\mathrm{C}_{2 / 444}$ interaction constant may occur between these sets of states belonging to the $\{11,2, u\}$ and $\{11,1, u\}$ polyads which are very close in energy, explaining the observed perturbations. For instance, two of the many interaction schemes contributing to the perturbations illustrated in Fig. 5, using the zero-order labels indicated on the figure, are:

$$
\begin{aligned}
& (10110) \stackrel{K_{3 / 245}}{\longrightarrow}(11021) \stackrel{C_{2 / 444}}{\longrightarrow}(10051) \\
& (10110) \stackrel{K_{1 / 255}}{\longrightarrow}(01112) \stackrel{C_{2 / 444}}{\longrightarrow}(00142)
\end{aligned}
$$

The composition of the eigenvectors of interest is very complicated and not detailed here. 


\section{Conclusion}

Thanks to the ultra high sensitivity of CW-CRDS, various new combination and hot bands could be recorded and analyzed, while additional $J$-lines were assigned in known bands, in the ranges $6000-6356 \mathrm{~cm}^{-1}$ and 6667-7015 $\mathrm{cm}^{-1}$ for ${ }^{12} \mathrm{C}_{2} \mathrm{H}_{2}$. These data were merged into a data base with all absorption and Raman vibration-rotation lines available from the literature accessing vibrational states up to $8600 \mathrm{~cm}^{-1}$. This data base amounts to 12137 transitions involving 186 different $k$ sub-states, counting $e$ and $f$ parities separately. All transitions were simultaneously fitted to $\left\{N_{r}, N_{s}, J, e / f, u / g\right\}$ Hamiltonian matrices, exploiting the vibrational polyad or cluster blockdiagonalization in acetylene. The dimensionless standard deviation of the fit was 0.92 , and 266 effective vibration-rotation parameters have been determined. Their quality has been assessed by various predictions and comparisons.

The present results strongly confirm the validity of the global approach to deal with the vibration-rotation structure in acetylene. The results also confirm the role of high order Coriolis type vibration-rotation interaction, probably affecting less than $2 \%$ of the data, not accounted for in the model and computer programs, yet.

The next steps in the acetylene saga ${ }^{7}$, will consist in additional spectral assignments in the range presently considered and resulting updates 
of the global constants. Higher excited ranges need also to be included in the fitting procedure. Finally, high order Coriolis type vibration-rotation interaction should be considered in more details.

\section{Acknowledgments}

We are most indebted to Dr. Perevalov (Tomsk) for providing an initial list of vibration-rotation data from the literature, and to Drs. G. Guelachvili and N. Picqué (U. Paris Sud) for providing their data set. S.R. thanks FRIA for financial support and the "Communauté française de Belgique " for a grant to travel to Bologna. This work was sponsored, in Italy, by the Università di Bologna and MIUR (PRIN «Trasferimenti di energia e di carica: dalle collisioni ai processi dissipativi ») and, in Belgium, by the Fonds National de la Recherche Scientifique (FNRS, contracts FRFC and IISN) and the «Action de Recherches Concertées de la Communauté française de Belgique ». It is performed within the "LEA HiRes " collaboration between ULB and UCL. This work was performed in the frame of the European research network QUASAAR (MRTN-CT-2004-512202). 


\section{References}

$1 \quad$ B. C. Smith and J. S. Winn, J. Chem. Phys. 89, 4638 (1988).

2 Q. Kou, G. Guelachvili, M. Abbouti Temsamani, and M. Herman, Can.

J. Phys. 72, 1241 (1994).

$3 \quad$ K. A. Keppler, G. C. Mellau, S. Klee, B. P. Winnewisser, M.

Winnewisser, J. Plíva, and K. N. Rao, J. Mol. Spectrosc. 175, 411 (1996).

4 H. Tran, J. Y. Mandin, V. Dana, L. Régalia-Jarlot, X. Thomas, and P. 
${ }_{12}$ P. Macko, D. Romanini, S. N. Mikhailenko, O. V. Naumenko, S. Kassi, A. Jenouvrier, V. G. Tyuterev, and A. Campargue, J. Mol. Spectrosc 227 (227), 90 (2004).

${ }^{13}$ Z. Majcherova, P. Macko, D. Romanini, V. Perevalov, S. A. Tashkun, J. L. Teffo, and A. Campargue, J. Mol. Spectrosc. 230, 1 (2005).

${ }_{14}$ B. V. Perevalov, S. Kassi, D. Romanini, V. I. Perevalov, S. A. Tashkun, and A. Campargue, J. Mol. Spectrosc., 241 (2006).

15 B. V. Perevalov, A. Campargue, B. Gao, S. Kassi, S. A. Tashkun, and V. I. Perevalov, J. Mol. Spectrosc. doi:10.1016/j.jms.2008.08.006 (2008).

16 A. Campargue, M.-R. De Backer-Barilly, A. Barbe, V. G. Tyuterev, and S. Kassi, Phys. Chem. Chem. Phys. 10, 2925 (2008).

${ }_{17}$ J. Morville, D. Romanini, A. A. Kachanov, and M. Chenevier, Appl. Phys. D 78, 465 (2004).

${ }_{18}$ L. S. Rothman, D. Jacquemart, A. Barbe, D. C. Benner, M. Birk, L. R. Brown, M. R. Carleer, C. J. Chakerian, K. V. Chance, V. Dana, V. M. Devi, J.-M. Flaud, R. R. Gamache, A. Goldman, J.-M. Hartmann, K. W. Jucks, A. Maki, J.-Y. Mandin, S. T. Massie, J. Orphal, A. Perrin, C. P. Rinsland, M. A. H. Smith, J. Tennyson, R. N. Tolchenov, R. A. Toth, J. Vander Auwera, P. Varanasi, and G. Wagner, J. Quant. Spectrosc. Radiative Transfer, 96, 139 (2005). R. Georges, M. Bach, and M. Herman, Mol. Phys. 90, 381 (1997). 
${ }^{20}$ Y. Kabbadj, M. Herman, G. Di Lonardo, L. Fusina, and J. W. C. Johns, J. Mol. Spectrosc. 150, 535 (1991).

${ }^{21}$ D. Bermejo, P. Cancio, G. Di Lonardo, and L. Fusina, J. Chem. Phys. 108, 7224 (1998).

${ }^{22}$ D. Bermejo, R. Z. Martinez, G. Di Lonardo, and L. Fusina, J. Chem. Phys. 111, 519 (1999).

${ }^{23} \quad$ D. Jacquemart, J.-Y. Mandin, V. Dana, L. Regalia-Jarlot, J.-J.

Plateaux, D. Decatoire, and L. S. Rothman, J. Quant. Spectrosc.

Radiative Transfer $\mathbf{7 6}, 237$ (2003).

Deleted: J.Q.S.R.T

${ }^{24}$ S. Robert, M. Herman, J. Vander Auwera, G. Di Lonardo, L. Fusina, G.

Blanquet, M. Lepère, and A. Fayt, Mol. Phys. 105, 559 (2007).

${ }^{25}$ K. F. Palmer, M. E. Mickelson, and K. N. Rao, J. Mol. Spectrosc. 44, 131 (1972).

${ }^{26}$ C. P. Rinsland, A. Baldacci, and K. N. Rao, Astrophys. J. Suppl. S. 49, 487 (1982).

${ }^{27}$ R. D'Cunha, Y. A. Sarma, V. A. Job, G. Guelachvili, and K. N. Rao, J. Mol. Spectrosc. 157, 358 (1993).

${ }_{28}$ Y. A. Sarma, R. D'Cunha, G. Guelachvili, R. Farrenq, and K. N. Rao, J. Mol. Spectrosc. 173, 561 (1995).

29

A. M. Tolonen and S. Alanko, Mol. Phys. 75, 1155 (1992). 
30 D. Jacquemart, J.-Y. Mandin, V. Dana, L. Regalia-Jarlot, X. Thomas, and P. Von der Heyden, J. Quant. Spectrosc. Radiative Transfer, 75, 397 (2002).

D. Jacquemart, J. Y. Mandin, V. Dana, C. Claveau, J. Vander Auwera, M. Herman, L. S. Rothman, L. Régalia-Jarlot, and A. Barbe, J. Quant.

\section{Spectrosc. Radiative Transfer 82, 363 (2003).}

Deleted: J.Q.S.R.T.

J. Y. Mandin, D. Jacquemart, V. Dana, L. Régalia-Jarlot, and A. Barbe,

\section{J. Quant. Spectrosc. Radiative Transfer 92, 239 (2005).}

Deleted: J.Q.S.R.T.

Formatted: French Belgium

${ }^{33}$ O. M. Lyulin, V. I. Perevalov, J.-Y. Mandin, V. Dana, D. Jacquemart,

L. Régalia-Jarlot, and A. Barbe, J. Quant. Spectrosc. Radiative

Transfer 97, 81 (2006).

Deleted: J.Q.S.R.T.

${ }_{34}$ D. Jacquemart, N. Lacome, J.-Y. Mandin, V. Dana, O. M. Lyulin, and

V. I. Perevalov, J. Quant. Spectrosc. Radiative Transfer, 103, 478

Deleted: J.Q.S.R.T. (2007).

O. M. Lyulin, V. I. Perevalov, J.-Y. Mandin, V. Dana, F. Gueye, X.

Thomas, P. Von der Heyden, D. Décatoire, L. Régalia-Jarlot, D.

Jacquemart, and N. Lacome, J. Quant. Spectrosc. Radiative Transfer, 103, 496 (2007).

Deleted: J.Q.S.R.T.

Formatted: French Belgium

Formatted: French Belgium

O. M. Lyulin, V. I. Perevalov, F. Gueye, J.-Y. Mandin, V. Dana, X.

Thomas, P. Von der Heyden, L. Régalia-Jarlot, and A. Barbe, J. Quant.

Deleted: J.Q.S.R.T. Spectrosc. Radiative Transfer, 104, 133 (2007). 
${ }^{37}$ O. M. Lyulin, D. Jacquemart, N. Lacome, V. I. Perevalov, and J.-Y.

Mandin, J. Quant. Spectrosc. Radiative Transfer, 109, 1856 (2008).

38 J. Vander Auwera, R. El Hachtouki, and L. R. Brown, Mol. Phys. 100, 3563 (2002).

${ }^{39}$ V. Girard, R. Farrenq, E. Sorokin, I. T. Sorokina, G. Guelachvili, and

N. Picqué, Chem. Phys. Lett. 419 (2006).

${ }^{40}$ S. A. B. Solina, J. P. O'Brien, R. W. Field, and W. F. Polik, J. Phys.

Chem. 100, 7797 (1996).

${ }^{41}$ M. Abbouti Temsamani and M. Herman, J. Chem. Phys. 102, 6371 (1995).

${ }^{42}$ M. Herman, M. I. El Idrissi, A. Pisarchik, A. Campargue, A.-C. Gaillot, L. Biennier, G. Di Lonardo, and L. Fusina, J. Chem. Phys. 108, 1377 (1998).

${ }^{43}$ M. I. El Idrissi, J. Liévin, A. Campargue, and M. Herman, J. Chem. Phys. 110, 2074 (1999).

${ }^{44}$ G. Di Lonardo, L. Fusina, E. Venuti, J. W. C. Johns, M. I. El Idrissi, J. Liévin, and M. Herman, J. Chem. Phys. 111, 1008 (1999).

${ }_{45}$ M. Herman, C. Depiesse, G. Di Lonardo, A. Fayt, L. Fusina, D. Hurtmans, S. Kassi, M. Mollabashi, and J. Vander Auwera, J. Mol. Spectrosc. 228, 499 (2004).

${ }^{46}$ S. Robert, A. Fayt, G. Di Lonardo, L. Fusina, F. Tamassia, and M. Herman, J. Chem. Phys. 123, 174302/1 (2005). 
${ }^{47}$ M. Kelman, J. Chem. Phys. 93, 6630 (1990).

48 M. E. Kelman, Annu. Rev. Phys. Chem. 46, 395 (1995).

49 S. A. B. Solina, J. P. O'Brien, R. W. Field, and W. F. Polik, Ber.

Bunsenges. Phys. Chem. 99, 555 (1995).

${ }^{50}$ R. W. Field, J. P. O'Brien, M. P. Jacobson, S. A. B. Solina, W. F. Polik, and H. Ishikawa, Adv. Chem. Phys. 101, 463 (1997).

51 B. I. Zhilinskii, M. I. El Idrissi, and M. Herman, J. Chem. Phys. 113, 7885 (2000).

52 M. I. El Idrissi, B. Zhilinskii, P. P. Gaspard, and M. Herman, Mol. Phys. 101, 595 (2003).

53 V. Tyng and M. E. Kellman, Journal of Physical Chemistry B, 18859 (2006).

54 O. M. Lyulin, V. I. Perevalov, and J. L. Teffo, SPIE XIVth symposium, $5311(2004)$. V. I. Perevalov, S. A. Tashkun, O. M. Lyulin, and J.-L. Teffo, The NATO Programme for Security through Science, C: Environmental security, 139 (2006), A. Perrin, N.B. Sari-Zizi, and J. Demaison eds (Springer). J. M. Brown, J. T. Hougen, K. P. Huber, J. W. C. Johns, I. Kopp, H. Lefebvre-Brion, A. J. Merer, D. A. Ramsay, J. Rostas, and R. N. Zare, J. Mol. Spectrosc. 55, 500 (1975). 
57 C. Vigouroux, A. Fayt, A. Guarnieri, A. Huckauf, H. Burger, D. Lentz, and D. Preugschat, J. Mol. Spectrosc. 202, 1 (2000).

${ }_{58}$ A. Fayt, C. Vigouroux, F. Willaert, L. Margules, L. F. Constantin, J. Demaison, G. Pawelke, El Bachir Mkadmi, and H. Buerger, J. Mol. Struct. 695-696, 295 (2004).

${ }^{59} \quad$ K. K. Lehmann, J. Chem. Phys. 79, 1098 (1983).

${ }^{60}$ B. Raghavendra, A. Moudens, J. Thiévin, B. Rowe, R. Georges, A. Fayt, B. Amyay, S. Robert, and M. Herman, Chem. Phys. Lett., submitted for publication (2008).

${ }_{61}$ J. Vander Auwera, D. Hurtmans, M. Carleer, and M. Herman, J. Mol. Spectrosc. 157, 337 (1993). 


\section{Figure captions}

Figure 1: Overview of the acetylene spectrum between 5850 and $7200 \mathrm{~cm}^{-1}$. The spectrum was recorded by Fourier Transform Spectroscopy at ULB (Experimental conditions: Pressure $=120 \mathrm{hPa}$, Pathlength: $48 \mathrm{~m}$ ). The two spectral intervals presently studied by CW-CRDS are indicated. Note the broad absorption features due to acetone below $6050 \mathrm{~cm}^{-1}$.

Figure 2: CW-CRDS spectrum of acetylene showing very weak absorption lines near $6235 \mathrm{~cm}^{-1}$. The sample pressure was $667 \mathrm{~Pa}$. Three successive enlargements show the typical sensitivity achieved by CW-CRDS: the noise level corresponding to the minimum value of the absorption coefficient which can be detected is about $5 \times 10^{-10} \mathrm{~cm}^{-1}$.

Figure 3: CW-CRDS spectrum of acetylene near $6028 \mathrm{~cm}^{-1}$ showing the very weak $Q$ branch of the $v_{1}+v_{2}+2 v_{5}\left(\Sigma_{\mathrm{g}}^{+}\right) \leftarrow v_{5}\left(\Pi_{\mathrm{u}}\right)$ band centred at $6030.084 \mathrm{~cm}^{-1}$, superimposed with the $R$ branch of the $2 v_{1}\left(\Sigma_{\mathrm{g}}^{+}\right) \leftarrow v_{5}\left(\Pi_{\mathrm{u}}\right)$ band. The sample pressure was $2666 \mathrm{~Pa}$.

Figure 4: $Q$ branch of the $2 v_{2}+3 v_{5}\left(\Pi_{u}\right) \leftarrow$ G.S band newly observed by CWCRDS near $6093 \mathrm{~cm}^{-1}$. The sample pressure was $2666 \mathrm{~Pa}$. 
Figure 5: Reduced energy graph (in $\mathrm{cm}^{-1}$ ) defined as $E(v, r)-1.162540 J(J+1)+1.626510^{-6} J^{2}(J+1)^{2}$, presenting vibration-rotation states of $u$-symmetry in ${ }^{12} \mathrm{C}_{2} \mathrm{H}_{2}$, in a selected energy range. Only those relevant to the perturbation affecting the $\left(v_{1} v_{2} v_{3} v_{4} v_{5}, l_{4} l_{5}\right)=(10110,10)$ substate are assigned, using the dominant zero order state in the eigenvector composition at low $J$-values. Full and empty dots indicate unperturbed and perturbed observed levels, respectively. Full and dashed lines indicate $e$ - and $f$ - parity states, respectively. The color code is adapted to the sub-state symmetry: in red, $\Sigma$; in blue, $\Pi$; in pink , $\Delta$; in green $\Gamma$. 
1

2

3

4

5

6

\section{Table legends}

Table 1: Band centres $\left(\tilde{v}_{c}\right)$, vibrational term values $\left(G_{v}\right)$, rotational constants (in $\mathrm{cm}^{-1}$ ), number of fitted and assigned lines, and standard deviation of the fit $\left(\sigma_{\text {dines }}\right.$ in $\left.\mathrm{cm}^{-1}\right)$ in ${ }^{12} \mathrm{C}_{2} \mathrm{H}_{2}$ from the band-by-band analysis ${ }^{a}$.

Table 2: Summary of vibration-rotation bands in the ${ }^{12} \mathrm{C}_{2} \mathrm{H}_{2}$ database used for the global fit.

$\underline{\text { Table 3: }}$ : Calculated term value $G_{c}$, in $\mathrm{cm}^{-1}$, of all states included in the data base of ${ }^{12} \mathrm{C}_{2} \mathrm{H}_{2}$ used for the global fit.

Table 4: Terms in the Hamiltonian used to perform the global vibrationrotation fit in ${ }^{12} \mathrm{C}_{2} \mathrm{H}_{2}{ }^{\text {a }}$.

Table 5: Vibration-rotation parameters $\left(\right.$ in $\mathrm{cm}^{-1}$ ) with their uncertainties $(1 \sigma)$ for the global fit of ${ }^{12} \mathrm{C}_{2} \mathrm{H}_{2}$.

Table 6: Statistical survey of the quality of the vibration-rotation data of ${ }^{12} \mathrm{C}_{2} \mathrm{H}_{2}$ accounted for in the global fit.

Table 7: Comparison of selected parameters $\left(\right.$ in $\mathrm{cm}^{-1}$ ) determined for two acetylene isotopologues using similar procedures (see text).

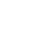


Table 8: Check of $x$-K relations in acetylene isotopologues_all parameters in $\underline{\left.\mathrm{cm}^{-1}\right)}$.

1

2

3

4

5

6

10

11

12

13

14

15

16

17

18

19

20

21

22

23

24

25

26

27

28

29

30

31

32

33

34

35

36

37

38

39

40

41

42

43

44

45

46

47

48

49

50

51

52

53

54

55

56

57

58

59

60 


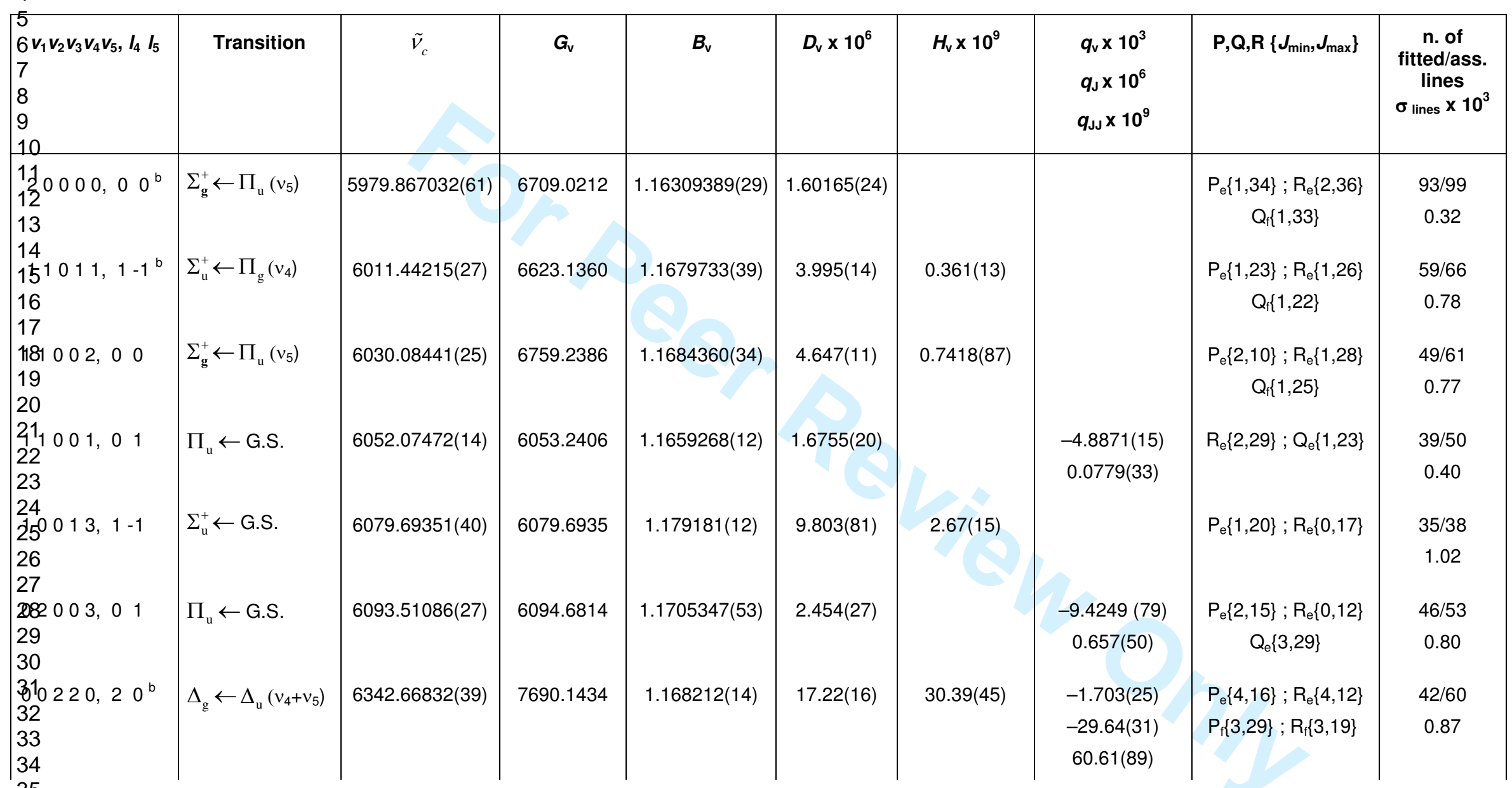




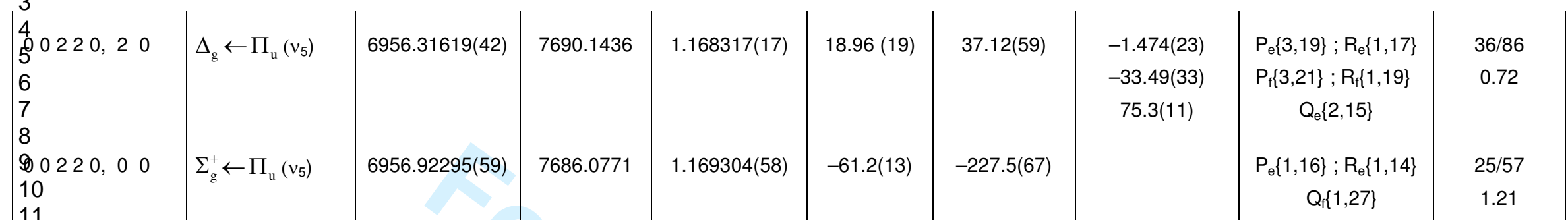

11

12

$13^{\text {a }}$ The vibrational assignments correspond to those of the dominant zero order state in the eigenvector composition at low $J$ -

14 values. The standard deviation $(1 \sigma)$ is indicated in parentheses in the unit of the last quoted digit. The assigned lines are listed for each branch.

15 The last column gives the number of lines included in the fit and the corresponding standard deviation. The band centre is

$17 \tilde{v}_{c}=G_{v}^{\prime}-B_{v}^{\prime} k^{\prime 2}-D_{v}^{\prime} k^{\prime 4}-G_{v}^{\prime \prime}+B_{v}^{\prime \prime 2} k^{\prime \prime 2}+D_{v}^{\prime \prime} k^{\prime \prime 4}$.

$18{ }^{\mathrm{b}}$ Band previously observed by Keppler et al. ${ }^{3}$. The range of $J$ values has been extended. 
Table 2

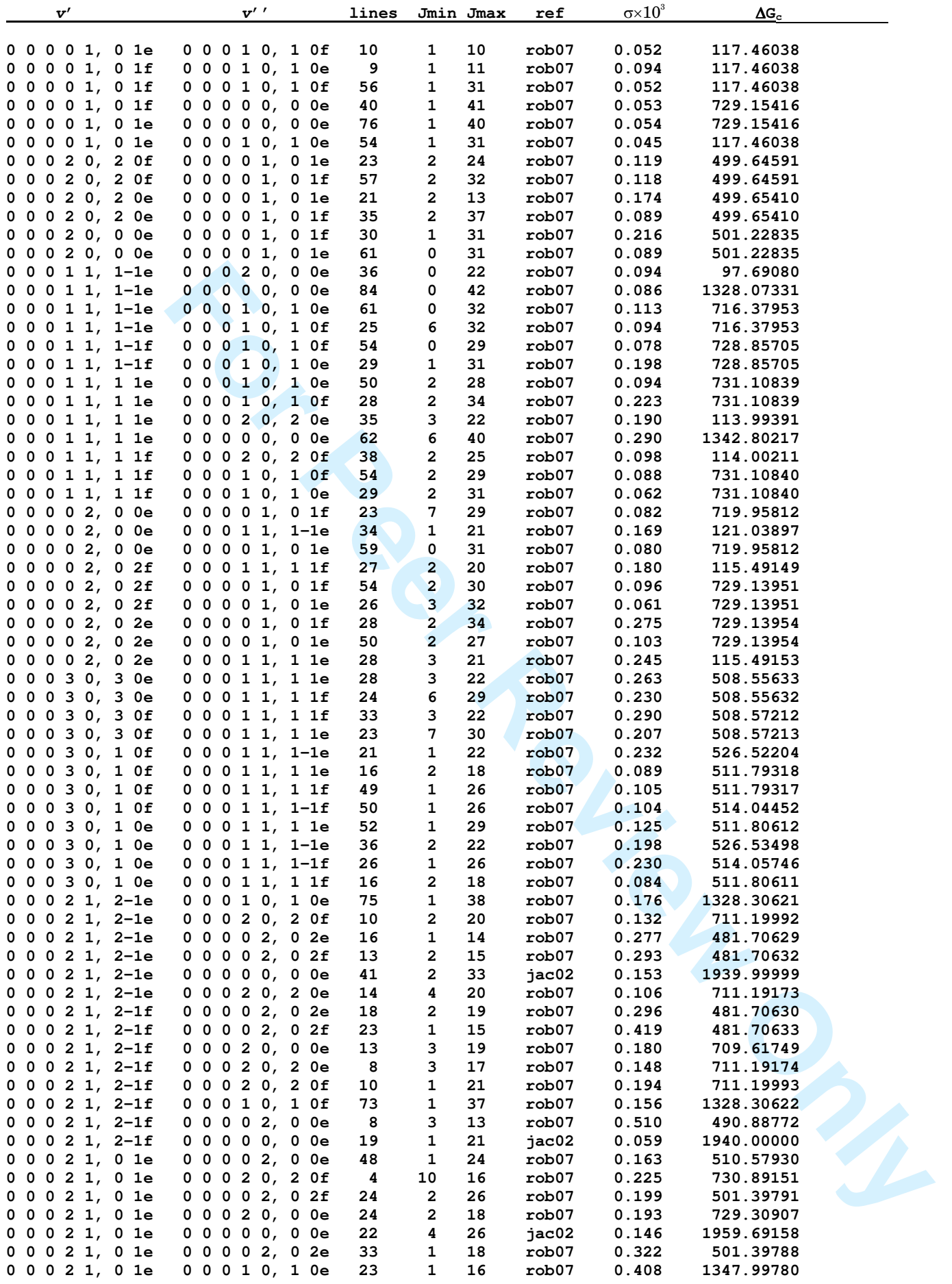


$\begin{array}{lllllll}0 & 0 & 0 & 2 & 1, & 0 & 1 \mathrm{f} \\ 0 & 0 & 0 & 2 & 1, & 0 & 1 \mathrm{f}\end{array}$

$\begin{array}{lllllllll}0 & 0 & 0 & 2 & 1, & 0 & 1 f\end{array}$

$\begin{array}{lllllll}0 & 0 & 0 & 2 & 1, & 0 & 1 f\end{array}$

$\begin{array}{llllllll}0 & 0 & 0 & 2 & 1, & 0 & 1 f\end{array}$

$\begin{array}{llllllll}0 & 0 & 0 & 2 & 1, & 0 & 1 f\end{array}$

$\begin{array}{lllllll}0 & 0 & 0 & 2 & 1, & 0 & 1 \mathrm{f}\end{array}$

$\begin{array}{lllllll}0 & 0 & 0 & 2 & 1, & 2 & 1 \mathrm{f}\end{array}$

$\begin{array}{lllllll}0 & 0 & 0 & 2 & 1,2 & 1 \mathrm{f}\end{array}$

$\begin{array}{lllllll}0 & 0 & 0 & 2 & 1,2 & 1 \mathrm{f}\end{array}$

$\begin{array}{lllllll}0 & 0 & 0 & 2 & 1,2 & 2 & 1 f\end{array}$

$\begin{array}{lllllll}0 & 0 & 0 & 2 & 1,2 & 1 \mathrm{f}\end{array}$

$\begin{array}{llllllll}0 & 0 & 0 & 2 & 1, & 2 & 1 e\end{array}$

$\begin{array}{lllllll}0 & 0 & 0 & 2 & 1,2 & 1 e\end{array}$

$\begin{array}{lllllll}0 & 0 & 0 & 2 & 1, & 2 & 1 e\end{array}$

$\begin{array}{lllllll}0 & 0 & 0 & 2 & 1, & 2 & 1 e\end{array}$

$\begin{array}{llllllllllllllllllllll}0 & 1 & 0 & 0 & 0 & 0 & 0 e\end{array}$

$\begin{array}{llllllllllll}0 & 1 & 0 & 0 & 0 & 0 & 0\end{array}$

$\begin{array}{llllllll}0 & 0 & 0 & 1 & 2, & 1 & 0 f\end{array}$

$\begin{array}{lllllll}0 & 0 & 0 & 1 & 2, & 1 & 0 f\end{array}$

$\begin{array}{lllllllllll}0 & 0 & 0 & 1 & 2, & 1 & 0\end{array}$

$\begin{array}{lllllll}0 & 0 & 0 & 1 & 2,-1 & 2 \mathrm{e}\end{array}$

$\begin{array}{llllllllll}0 & 0 & 0 & 1 & 2,-1 & 2 \mathrm{e}\end{array}$

$\begin{array}{llllllll}0 & 0 & 0 & 1 & 2,-1 & 2 f\end{array}$

$\begin{array}{lllllll}0 & 0 & 0 & 1 & 2,-1 & 2 \mathrm{f}\end{array}$

$\begin{array}{lllllll}0 & 0 & 0 & 1 & 2, & 1 & 2 e\end{array}$

$\begin{array}{lllllll}0 & 0 & 0 & 1 & 2, & 1 & 2 \mathrm{e}\end{array}$

$\begin{array}{lllllll}0 & 0 & 0 & 1 & 2, & 1 & 2 f\end{array}$

$\begin{array}{lllllll}0 & 0 & 0 & 1 & 2, & 1 & 2 f\end{array}$

$\begin{array}{lllllll}0 & 0 & 0 & 0 & 3, & 0 & 1 f\end{array}$

$\begin{array}{lllllll}0 & 0 & 0 & 0 & 3, & 0 & 1 \mathrm{f}\end{array}$

$\begin{array}{lllllll}0 & 0 & 0 & 0 & 3, & 0 & 1 \mathrm{f}\end{array}$

$\begin{array}{llllllll}0 & 0 & 0 & 0 & 3, & 0 & 1 \mathrm{f}\end{array}$

$\begin{array}{lllllll}0 & 0 & 0 & 0 & 3, & 0 & 1 e\end{array}$

$\begin{array}{lllllll}0 & 0 & 0 & 0 & 3, & 0 & 1 \mathrm{e}\end{array}$

$\begin{array}{lllllllllll}0 & 0 & 0 & 0 & 3, & 0 & 1 \mathrm{e}\end{array}$

$\begin{array}{lllllll}0 & 0 & 0 & 0 & 3, & 0 & 1 e\end{array}$

$\begin{array}{lllllllllllll}0 & 0 & 0 & 0 & 3, & 0 & 3 e\end{array}$

$\begin{array}{lllllll}0 & 0 & 0 & 0 & 3, & 0 & 3 f\end{array}$

$\begin{array}{lllllll}0 & 0 & 0 & 3 & 1,3-1 f\end{array}$

$\begin{array}{lllllll}0 & 0 & 0 & 3 & 1, & 3-1 f\end{array}$

$\begin{array}{lllllll}0 & 0 & 0 & 3 & 1,3-1 e\end{array}$

$\begin{array}{llllll}0 & 0 & 0 & 3 & 1,3-1 e\end{array}$

$\begin{array}{lllllll}0 & 0 & 0 & 3 & 1,1-1 \mathrm{e}\end{array}$

$\begin{array}{lllllll}0 & 0 & 0 & 3 & 1,1-1 e\end{array}$

$\begin{array}{llllllll}0 & 0 & 0 & 3 & 1,1-1 e\end{array}$

$\begin{array}{lllllllllll}0 & 1 & 0 & 1 & 0, & 0 \text { e }\end{array}$

$\begin{array}{llllllll}0 & 1 & 0 & 1 & 0, & 1 & 0 f\end{array}$

$\begin{array}{lllllll}0 & 0 & 0 & 3 & 1,1-1 f\end{array}$

$\begin{array}{lllllll}0 & 0 & 0 & 3 & 1,1-1 f\end{array}$

$\begin{array}{lllllll}0 & 0 & 0 & 3 & 1, & 1 & 1 \mathrm{f}\end{array}$

$\begin{array}{llllllllll}0 & 0 & 0 & 3 & 1, & 1 \mathrm{e}\end{array}$

$\begin{array}{lllllll}0 & 0 & 0 & 2 & 2, & 2-2 e\end{array}$

$\begin{array}{lllllll}0 & 0 & 0 & 2 & 2, & 2-2\end{array}$

$\begin{array}{llllll}0 & 0 & 0 & 2 & 2, & 2-2 \mathrm{e}\end{array}$

$\begin{array}{llllll}0 & 0 & 0 & 2 & 2, & 2-2 f\end{array}$

$\begin{array}{lllllll}0 & 0 & 0 & 2 & 2, & 2-2 f\end{array}$

$\begin{array}{lllllll}0 & 0 & 0 & 2 & 2, & 2-2 f\end{array}$

$\begin{array}{lllllll}0 & 0 & 0 & 2 & 2, & 2 & 0 f\end{array}$

$\begin{array}{lllllll}0 & 0 & 0 & 2 & 2, & 2 & 0 f\end{array}$

$\begin{array}{lllllll}0 & 0 & 0 & 2 & 2, & 2 & 0 f\end{array}$

$\begin{array}{lllllll}0 & 0 & 0 & 2 & 2, & 2 & 0\end{array}$

$\begin{array}{lllllll}0 & 1 & 0 & 0 & 1, & 0 & 1 e\end{array}$

$\begin{array}{llllllll}0 & 1 & 0 & 0 & 1, & 0 & 1 \mathrm{f}\end{array}$

$\begin{array}{llllllllllll}0 & 1 & 0 & 0 & 1, & 0 & 1 e\end{array}$

$\begin{array}{lllllllll}0 & 1 & 0 & 0 & 1, & 0 & 1 \mathrm{f}\end{array}$

$\begin{array}{llllll}0 & 0 & 0 & 1 & 3, & 1-1 e\end{array}$

$\begin{array}{llllll}0 & 0 & 0 & 1 & 3, & 1-1 \mathrm{e}\end{array}$

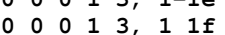

$\begin{array}{llllllll}0 & 0 & 0 & 0 & 2, & 0 & 2 \mathrm{f}\end{array}$

$\begin{array}{llllllllllll}0 & 0 & 0 & 0 & 2 & 0 & 2 e\end{array}$

$\begin{array}{lllllll}0 & 0 & 2 & 0,2 & 0 e\end{array}$

$\begin{array}{llllllll}0 & 0 & 0 & 1 & 0, & 1 & 0 f\end{array}$

$\begin{array}{lllllllllll}0 & 0 & 0 & 0 & 0, & 0 & 0 e\end{array}$

$\begin{array}{llllllll}0 & 0 & 0 & 2 & 0, & 0 & 0\end{array}$

$\begin{array}{llllllll}0 & 0 & 0 & 0 & 2, & 0 & 0 e\end{array}$

$\begin{array}{lllllll}0 & 0 & 0 & 1 & 0, & 1 & 0 f\end{array}$

$\begin{array}{llllllll}0 & 0 & 0 & 2 & 0, & 2 & 0 e\end{array}$

$\begin{array}{llllllll}0 & 0 & 0 & 2 & 0, & 2 & 0 f\end{array}$

$\begin{array}{llllllll}0 & 0 & 0 & 0 & 2, & 0 & 2 e\end{array}$

$\begin{array}{llllllll}0 & 0 & 0 & 0 & 2, & 0 & 2 f\end{array}$

$\begin{array}{llllllll}0 & 0 & 0 & 0 & 2, & 0 & 2 e\end{array}$

$\begin{array}{llllllll}0 & 0 & 0 & 2 & 0, & 2 & 0\end{array}$

$\begin{array}{lllllll}0 & 0 & 0 & 2 & 0, & 2 & \text { of }\end{array}$

$\begin{array}{llllllll}0 & 0 & 0 & 0 & 2, & 0 & 2 f\end{array}$

$\begin{array}{llllllll}0 & 0 & 0 & 0 & 1, & 0 & 1 \mathrm{f}\end{array}$

$\begin{array}{lllllllllllll}0 & 0 & 0 & 0 & 1, & 0 & 1 e\end{array}$

$\begin{array}{lllllll}0 & 0 & 0 & 1 & 1,1-1 e\end{array}$

$\begin{array}{llllllll}0 & 0 & 0 & 0 & 1, & 0 & 1 \mathrm{f}\end{array}$

$\begin{array}{llllllll}0 & 0 & 0 & 1 & 1,1-1 e\end{array}$

$\begin{array}{lllllllllll}0 & 0 & 0 & 0 & 1, & 0 & 1 e\end{array}$

$\begin{array}{lllllll}0 & 0 & 0 & 1 & 1,1-1 f\end{array}$

$\begin{array}{llllllllll}0 & 0 & 0 & 0 & 1, & 0 & 1 e\end{array}$

$\begin{array}{lllllllll}0 & 0 & 0 & 0 & 1, & 0 & 1 \mathrm{f}\end{array}$

$0 \begin{array}{lllllll}0 & 0 & 1 & 1, & 1-1 \mathrm{f}\end{array}$

$\begin{array}{llllllll}0 & 0 & 0 & 1 & 1, & 1 & 1 e\end{array}$

$\begin{array}{llllllll}0 & 0 & 0 & 1 & 1, & 1 & 1 f\end{array}$

$\begin{array}{llllllll}0 & 0 & 0 & 1 & 1, & 1 & 1 \mathrm{f}\end{array}$

$\begin{array}{lllllll}0 & 0 & 1 & 1, & 1 & 1 e\end{array}$

$\begin{array}{llllllll}0 & 0 & 0 & 0 & 2, & 0 & 2 \mathrm{f}\end{array}$

$\begin{array}{llllllllllll}0 & 0 & 0 & 0 & 2, & 0 & 2 e\end{array}$

$\begin{array}{llllllllllll}0 & 0 & 0 & 0 & 2, & 0 & 0 e\end{array}$

$\begin{array}{llllllllll}0 & 0 & 0 & 0 & 0, & 0 & 0 e\end{array}$

$\begin{array}{llllllll}0 & 0 & 0 & 0 & 2, & 0 & 2 f\end{array}$

$\begin{array}{llllllllllll}0 & 0 & 0 & 0 & 2, & 0 & 2 e\end{array}$

$\begin{array}{llllllll}0 & 0 & 0 & 0 & 2, & 0 & 0 \mathrm{e}\end{array}$

$\begin{array}{llllllllllll}0 & 0 & 0 & 0 & 0, & 0 & 0 e\end{array}$

$\begin{array}{llllllll}0 & 0 & 0 & 0 & 2, & 0 & 2 e\end{array}$

$\begin{array}{lllllll}0 & 0 & 0 & 0 & 2, & 0 & 2 f\end{array}$

$\begin{array}{lllllllllllll}0 & 0 & 0 & 1 & 0, & 1 & 0 f\end{array}$

$\begin{array}{lllllll}0 & 0 & 0 & 2 & 0, & 2 & 0 f\end{array}$

$\begin{array}{llllllllllllllllll}0 & 0 & 0 & 1 & 0, & 1 & 0\end{array}$

$\begin{array}{lllllllllll}0 & 0 & 0 & 2 & 0,2 & 0 \mathrm{e}\end{array}$

$\begin{array}{llllllllllll}0 & 0 & 0 & 0 & 0, & 0 & 0 e\end{array}$

$\begin{array}{llllllll}0 & 0 & 0 & 2 & 0, & 0 & 0 e\end{array}$

$\begin{array}{llllllllllllllllllll}0 & 0 & 0 & 1 & 0, & 1 & 0\end{array}$

$\begin{array}{lllllllllll}0 & 0 & 0 & 0 & 1, & 0 & 1 \mathrm{e}\end{array}$

$\begin{array}{llllllll}0 & 0 & 0 & 0 & 1, & 0 & 1 \mathrm{f}\end{array}$

$\begin{array}{llllllll}0 & 0 & 0 & 1 & 0, & 1 & 0 f\end{array}$

$\begin{array}{llllllllllll}0 & 0 & 0 & 1 & 0, & 1 & 0\end{array}$

$\begin{array}{lllllll}0 & 0 & 0 & 1 & 0, & 1 & \text { of }\end{array}$

$\begin{array}{lllllllllll}0 & 0 & 0 & 1 & 0, & 1 & 0 e\end{array}$

$\begin{array}{llllllll}0 & 0 & 0 & 0 & 1, & 0 & 1 \mathrm{f}\end{array}$

$\begin{array}{lllllllllll}0 & 0 & 0 & 0 & 1, & 0 & 1 e\end{array}$

$0 \begin{array}{llllll}0 & 0 & 0 & 1 & 1, & 1-1 \mathrm{e}\end{array}$

$\begin{array}{llllllll}0 & 0 & 0 & 1 & 1, & 1-1 \mathrm{f}\end{array}$

$\begin{array}{llllllll}0 & 0 & 0 & 1 & 1, & 1 & 1 \mathrm{f}\end{array}$

$\begin{array}{llllllll}0 & 0 & 0 & 0 & 1, & 0 & 1 f\end{array}$

$\begin{array}{llllllll}0 & 0 & 0 & 0 & 1, & 0 & 1 \mathrm{f}\end{array}$

$\begin{array}{llllllll}0 & 0 & 0 & 1 & 1, & 1 & 1 \mathrm{f}\end{array}$

$0 \begin{array}{lllllll}0 & 0 & 0 & 1 & 1,1-1 f\end{array}$

$\begin{array}{llllllllll}0 & 0 & 0 & 1 & 1, & 1 & 1 e\end{array}$

$\begin{array}{llllllllllllllllll}0 & 0 & 0 & 0 & 0, & 0 & 0 e\end{array}$

$\begin{array}{lllllllllllllllll}0 & 0 & 0 & 0 & 0, & 0 & 0 \mathrm{e}\end{array}$

$\begin{array}{lllllllllll}0 & 0 & 0 & 1 & 0, & 1 & 0 e\end{array}$

$\begin{array}{llllllll}0 & 0 & 0 & 1 & 0, & 1 & 0 f\end{array}$

$\begin{array}{llllllllllllllllll}0 & 0 & 0 & 0 & 2, & 0 & 0 e\end{array}$

$\begin{array}{lllllll}0 & 0 & 0 & 1 & 0, & 1 & 0 f\end{array}$

$\begin{array}{llllllllllll}0 & 0 & 0 & 1 & 0, & 1 & 0 \mathrm{e}\end{array}$

$\begin{array}{lllllll}0 & 0 & 0 & 1 & 0, & 1 & 0 f\end{array}$

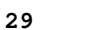

$\begin{array}{rr}2 & 23 \\ 13 & 15 \\ 1 & 27\end{array}$

10

141

$\begin{array}{rr}1 & 22 \\ 13 & 29\end{array}$

313

31

$3 \quad 12$

$3 \quad 27$

326

16

20

$3 \quad 20$

27

$\begin{array}{ll}0 & 25 \\ 2 & 24\end{array}$

138

$3 \quad 21$

37

23

$\begin{array}{ll}1 & 29 \\ 1 & 28\end{array}$

20

$\begin{array}{ll}4 & 20 \\ 3 & 19\end{array}$

323

420

$4 \quad 18$

7

17

17
21
35

35

$8 \quad 8$

120

36

322

$\begin{array}{ll}5 & 21 \\ 5 & 25\end{array}$

231

426

$\begin{array}{ll}3 & 32 \\ 0 & 20\end{array}$

030

$20 \quad 20$

$1 \quad 19$

$\begin{array}{ll}1 & 22 \\ 7 & 11\end{array}$

$\begin{array}{rr}4 & 13 \\ 10 & 12\end{array}$

$\begin{array}{ll}10 & 12 \\ 10 & 11\end{array}$

52

$\begin{array}{ll}5 & 19 \\ 0 & 3\end{array}$

$\begin{array}{ll}0 & 31 \\ 0 & 24\end{array}$

$10 \quad 24$

$\begin{array}{rr}8 & 22 \\ 4 & 28\end{array}$

428

12
4 29

$\begin{array}{ll}4 & 29 \\ 1 & 23\end{array}$

125

120

120

026

$1 \quad 18$

21
$4 \quad 22$

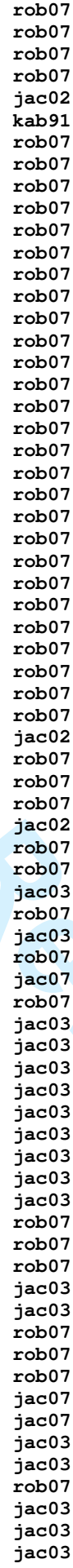

0.126

0.170

0.000

0.385

0.052

0.000

0.066

0.284

150

0.130

0.472

0.196

0.246

0.168

0.263

0.123

0.312

0.149

0.206

0.111

0.111

0.184

0.327

0.308

0.188

0.238

0.123

0.164

0.225

0.127

0.214

0.153

0.111

0.065

0.092

0.200

0.061

0.237

0.248

0.133

0.314

0.090

0.351

0.076

0.189

0.113

0.159

0.100

0.263

0.198

0.138

0.082

0.186

0.075

0.289

0.242

.333

0.133

0.484

0.271

0.331

0.279

0.053

0.092

0.204

0.148

0.236

0.197

0.153

0.201

1959.69197

729.30946 


\begin{tabular}{|c|c|c|c|c|c|c|c|c|c|c|c|c|c|c|c|c|c|c|}
\hline 0 & 0 & 01 & 1 & 3, & $11 \mathrm{f}$ & 0 & 0 & 0 & 0 & 2, & 0 & $2 f$ & 44 & 2 & 27 & rob07 & 0.342 & 1310.15665 \\
\hline 0 & 0 & 01 & 13 & 3, & $11 \mathrm{f}$ & 0 & 0 & 0 & 1 & 0 & 1 & $0 e$ & 13 & 3 & 21 & jac03 & 0.199 & 2156.75654 \\
\hline 0 & 0 & 01 & 13 & 3, & $11 \mathrm{e}$ & 0 & 0 & 0 & 1 & 0 & 1 & $0 e$ & 14 & 4 & 22 & jac03 & 0.221 & 2156.75745 \\
\hline 0 & 0 & 01 & 13 & 3, & $11 \mathrm{e}$ & 0 & 0 & 0 & 1 & 0 & 1 & $0 f$ & 13 & 2 & 19 & jac03 & 0.118 & 2156.75745 \\
\hline 0 & 0 & 01 & 13 & 3, & $11 \mathrm{e}$ & 0 & 0 & 0 & 0 & 2 & 0 & $2 e$ & 46 & 2 & 26 & rob07 & 0.359 & 1310.15753 \\
\hline 0 & 0 & 01 & 13 & 3, & $1-1 \mathrm{f}$ & 0 & 0 & 0 & 1 & 0 & 1 & $0 f$ & 28 & 4 & 25 & jac03 & 0.171 & 2171.95625 \\
\hline 0 & 0 & 01 & 13 & 3, & $1-1 f$ & 0 & 0 & 0 & 1 & 0 & 1 & $0 e$ & 13 & 4 & 22 & jac03 & 0.168 & 2171.95625 \\
\hline 0 & 0 & 01 & 13 & $3,-$ & $-13 e$ & 0 & 0 & 0 & 1 & 0 & 1 & $0 f$ & 20 & 3 & 27 & jac03 & 0.237 & 2179.06955 \\
\hline 0 & 0 & 01 & 13 & $3,-$ & $-13 e$ & 0 & 0 & 0 & 1 & 0 & 1 & $0 e$ & 15 & 5 & 16 & jac03 & 0.155 & 2179.06955 \\
\hline 0 & 0 & 01 & 13 & $3,-$ & $-13 f$ & 0 & 0 & 0 & 1 & 0 & 1 & $0 e$ & 18 & 2 & 24 & jac03 & 0.142 & 2179.06972 \\
\hline 0 & 0 & 01 & 13 & $3,-$ & $-13 f$ & 0 & 0 & 0 & 1 & 0 & 1 & $0 f$ & 14 & 3 & 18 & jac03 & 0.168 & 2179.06972 \\
\hline 0 & 0 & 00 & 04 & 4, & $00 e$ & 0 & 0 & 0 & 0 & 1, & 0 & $1 e$ & 28 & 0 & 28 & jac03 & 0.160 & 2151.06475 \\
\hline 0 & 0 & 00 & 04 & 4, & $00 e$ & 0 & 0 & 0 & 0 & 1, & 0 & $1 \mathrm{f}$ & 7 & 1 & 24 & jac03 & 0.121 & 2151.06475 \\
\hline 0 & 0 & 0 & 04 & 4, & $02 f$ & 0 & 0 & 0 & 0 & 1, & 0 & $1 \mathrm{f}$ & 27 & 4 & 25 & jac03 & 0.101 & 2160.16967 \\
\hline 0 & 0 & 00 & 04 & 4, & $02 f$ & 0 & 0 & 0 & 0 & 1, & 0 & $1 e$ & 13 & 3 & 26 & jac03 & 0.175 & 2160.16967 \\
\hline 0 & 0 & 00 & 04 & 4, & $02 e$ & 0 & 0 & 0 & 0 & 1, & 0 & $1 \mathrm{f}$ & 15 & 2 & 26 & jac03 & 0.132 & 2160.17108 \\
\hline 0 & 0 & 00 & 04 & 4, & $02 e$ & 0 & 0 & 0 & 0 & 1, & 0 & $1 e$ & 21 & 5 & 22 & jac03 & 0.147 & 2160.17108 \\
\hline 0 & 0 & 10 & 00 & 0, & $00 e$ & 0 & 0 & 0 & 0 & 0 & 0 & $0 \mathrm{e}$ & 65 & 0 & 32 & vda93 & 0.195 & 3281.89920 \\
\hline 0 & 0 & 1 & 00 & 0, & $00 e$ & 0 & 0 & 0 & 1 & 0, & 1 & $0 f$ & 33 & 1 & 33 & vda93 & 0.155 & 2670.20542 \\
\hline 0 & 0 & 1 & 00 & 0, & $00 e$ & 0 & 0 & 0 & 1 & 0, & 1 & $0 \mathrm{e}$ & 68 & 0 & 36 & vda93 & 0.204 & 2670.20542 \\
\hline 0 & 1 & 0 & 11 & 1, & $1-1 e$ & 0 & 0 & 0 & 1 & 0, & 1 & $0 \mathrm{e}$ & 68 & 0 & 36 & vda93 & 0.208 & 2683.14582 \\
\hline 0 & 1 & 0 & 1 & 1, & $1-1 e$ & 0 & 0 & 0 & 0 & 0, & 0 & $0 \mathrm{e}$ & 66 & 0 & 36 & vda93 & 0.185 & 3294.83960 \\
\hline 0 & 1 & 0 & 1 & 1, & $1-1 e$ & 0 & 0 & 0 & 1 & 0 & 1 & $0 f$ & 34 & 1 & 34 & vda93 & 0.429 & 2683.14582 \\
\hline 0 & 1 & 0 & 1 & 1, & $1-1 \mathrm{f}$ & 0 & 0 & 0 & 1 & 0 & 1 & $0 \mathrm{e}$ & 28 & 1 & 29 & vda93 & 0.526 & 2688.94121 \\
\hline 0 & 1 & 0 & 1 & 1, & $1-1 f$ & 0 & 0 & 0 & 1 & 0 , & 1 & $0 f$ & 45 & 0 & 27 & vda93 & 0.552 & 2688.94121 \\
\hline 0 & 1 & 0 & 1 & 1, & $11 \mathrm{f}$ & 0 & 0 & 0 & 1 & 0 & 1 & $0 f$ & 47 & 2 & 29 & vda93 & 0.642 & 2691.32364 \\
\hline 0 & 1 & 0 & 1 & 1, & $11 \mathrm{f}$ & 0 & 0 & 0 & 1 & 0, & 1 & $0 \mathrm{e}$ & 25 & 2 & 28 & vda93 & 0.505 & 2691.32364 \\
\hline 0 & 1 & 0 & 1 & 1, & $11 \mathrm{e}$ & 0 & 0 & 0 & 1 & 0 & 1 & $0 e$ & 32 & 2 & 22 & vda93 & 0.582 & 2691.32367 \\
\hline 0 & 1 & 0 & 1 & 1, & $11 \mathrm{e}$ & 0 & 0 & 0 & 1 & 0 & 1 & $0 f$ & 23 & 2 & 26 & vda93 & 0.453 & 2691.32367 \\
\hline 1 & 0 & 0 & 00 & 0 & 00 e & 0 & 0 & 0 & 0 & 1 & 0 & $1 \mathrm{f}$ & 30 & 1 & 36 & $\begin{array}{l}\text { pal72 } \\
\text { jac07 }\end{array}$ & 0.156 & 2643.69309 \\
\hline 1 & 0 & 0 & 00 & 0 , & $00 e$ & 0 & 0 & 0 & 0 & 1 & 0 & $1 e$ & 62 & 0 & 37 & $\begin{array}{l}\text { pal72 } \\
\text { jac07 }\end{array}$ & 0.155 & 2643.69309 \\
\hline 0 & 1 & 02 & 21 & 1, & $2-1 f$ & 0 & 0 & 0 & 1 & 0 & 1 & $0 f$ & 43 & 2 & 33 & $\operatorname{man} 05$ & 0.282 & 3269.53953 \\
\hline 0 & 1 & 0 & 21 & 1, & $2-1 e$ & 0 & 0 & 0 & 0 & 0 & 0 & $0 e$ & 76 & 1 & 44 & lyu07 & 0.256 & 3881.23331 \\
\hline 0 & 1 & 0 & 21 & 1, & $2-1 e$ & 0 & 0 & 0 & 1 & 0 & 1 & $0 e$ & 40 & 2 & 32 & $\operatorname{man} 05$ & 0.161 & 3269.53953 \\
\hline 0 & 0 & 1 & 10 & 0, & $10 e$ & 0 & 0 & 0 & 1 & 0 & 1 & $0 e$ & 39 & 1 & 32 & $\operatorname{man} 05$ & 0.142 & 3285.46885 \\
\hline 0 & 0 & 1 & 1 & 0 & $10 e$ & 0 & 0 & 0 & 0 & 0 & 0 & $0 e$ & 70 & 1 & 42 & $\begin{array}{l}\text { lyu07 } \\
\text { gir06 }\end{array}$ & 0.269 & 3897.16263 \\
\hline 0 & 0 & 1 & 10 & 0, & $10 f$ & 0 & 0 & 0 & 0 & 0 & 0 & $0 e$ & 18 & 19 & 41 & lyu07 & 0.103 & 3897.16265 \\
\hline 0 & 0 & 1 & 10 & 0 , & $10 f$ & 0 & 0 & 0 & 1 & 0 & 1 & $0 f$ & 40 & 1 & 31 & $\operatorname{man} 05$ & 0.188 & 3285.46887 \\
\hline 0 & 1 & 0 & 21 & 1, & $01 e$ & 0 & 0 & 0 & 0 & 0 & 0 & $0 e$ & 14 & 12 & 30 & gir06 & 0.464 & 3908.13474 \\
\hline 0 & 2 & 0 & 00 & 0, & $00 \mathrm{e}$ & 0 & 1 & 0 & 0 & 0 & 0 & $0 e$ & 19 & 1 & 19 & ber98 & 0.449 & 1959.58152 \\
\hline 1 & 0 & 0 & 1 & 0 , & 1 of & 0 & 0 & 0 & 0 & 1 & 0 & $1 \mathrm{f}$ & 52 & 1 & 27 & sar95 & 0.738 & 3239.72968 \\
\hline 1 & 0 & 0 & 1 & 0 , & $10 e$ & 0 & 0 & 0 & 0 & 1, & 0 & $1 e$ & 49 & 1 & 26 & sar95 & 0.644 & 3239.72968 \\
\hline 0 & 0 & 1 & 0 & 1, & $01 e$ & * 0 & 0 & 0 & 0 & 1, & 0 & $1 e$ & 32 & 1 & 31 & $\operatorname{man} 05$ & 0.174 & 3272.10560 \\
\hline 0 & 0 & 1 & 0 & 1, & $01 \mathrm{f}$ & * & 0 & 0 & 0 & 1, & 0 & $1 \mathrm{f}$ & 38 & 1 & 28 & $\operatorname{man} 05$ & 0.169 & 3272.10561 \\
\hline 0 & 0 & 1 & 0 & 1, & $01 \mathrm{f}$ & * & 0 & 0 & 0 & 1, & 0 & $1 \mathrm{f}$ & 30 & 4 & 24 & $\operatorname{man} 05$ & 0.172 & 3286.38202 \\
\hline 0 & 0 & 1 & 01 & 1, & $01 e$ & * 0 & 0 & 0 & 0 & 1, & 0 & $1 e$ & 27 & 5 & 27 & $\operatorname{man} 05$ & 0.271 & 3286.38203 \\
\hline 1 & 0 & 0 & 01 & 1, & $01 \mathrm{f}$ & 0 & 0 & 0 & 1 & 0 & 1 & $0 f$ & 53 & 1 & 28 & sar95 & 0.815 & 3479.47881 \\
\hline 1 & 0 & 0 & 01 & 1, & $01 e$ & 0 & 0 & 0 & 1 & 0 & 1 & $0 e$ & 53 & 1 & 28 & sar95 & 0.542 & 3479.47881 \\
\hline 1 & 0 & 0 & 01 & 1, & $01 \mathrm{e}$ & 0 & 0 & 0 & 0 & 0 & 0 & $0 e$ & 80 & 1 & 46 & $\begin{array}{l}\text { dcu91 } \\
\text { gir06 } \\
\text { lyu07 }\end{array}$ & 0.362 & 4091.17259 \\
\hline 1 & 0 & 0 & 01 & 1, & $01 \mathrm{f}$ & 0 & 0 & 0 & 0 & 0 & 0 & $0 e$ & 38 & 1 & 38 & $\begin{array}{l}\text { dcu91 } \\
\text { lyu07 }\end{array}$ & 0.286 & 4091.17259 \\
\hline 0 & 1 & 0 & 03 & 3, & $01 \mathrm{f}$ & 0 & 0 & 0 & 0 & 0 & 0 & $0 e$ & 26 & 1 & 27 & $\begin{array}{l}\text { dcu91 } \\
\text { lyu07 }\end{array}$ & 0.750 & 4138.87768 \\
\hline 0 & 1 & 0 & 03 & 3, & $01 \mathrm{e}$ & 0 & 0 & 0 & 0 & 0 & 0 & $0 e$ & 54 & 1 & 32 & $\begin{array}{l}\text { dcu91 } \\
\text { lyu07 }\end{array}$ & 0.379 & 4138.87771 \\
\hline 0 & 1 & 03 & 31 & 1, & $3-1 f$ & 0 & 0 & 0 & 2 & 0 & 2 & $0 f$ & 51 & 3 & 35 & $\begin{array}{l}\text { rin82 } \\
\text { lyu06 }\end{array}$ & 2.090 & 3257.31679 \\
\hline 0 & 1 & 03 & 31 & 1, & $3-1 e$ & 0 & 0 & 0 & 2 & 0 & 2 & $0 e$ & 53 & 3 & 36 & $\begin{array}{l}\text { rin82 } \\
\text { lyu06 }\end{array}$ & 1.297 & 3257.31166 \\
\hline 0 & 1 & 03 & 31 & 1, & $1-1 e$ & 0 & 0 & 0 & 2 & 0 & 0 & $0 e$ & 51 & 2 & 38 & $\begin{array}{l}\text { rin82 } \\
\text { lyu06 }\end{array}$ & 1.512 & 3258.45564 \\
\hline 0 & 1 & 0 & 31 & 1, & $1-1 e$ & 0 & 0 & 0 & 0 & 0 & 0 & $0 e$ & 58 & 0 & 31 & lyu $7 b$ & 0.178 & 4488.83815 \\
\hline 0 & 0 & 1 & 20 & 0 & $20 e$ & 0 & 0 & 0 & 1 & 0 & 1 & $0 e$ & 52 & 2 & 31 & $\begin{array}{l}\text { sar95 } \\
\text { gir06 }\end{array}$ & 0.451 & 3892.84265 \\
\hline 0 & 0 & 1 & 20 & 0, & $20 e$ & 0 & 0 & 0 & 1 & 0 & 1 & $0 f$ & 19 & 2 & 20 & sar95 & 0.418 & 3892.84265 \\
\hline 0 & 0 & 1 & 20 & 0 & $20 e$ & 0 & 0 & 0 & 2 & 0 & 2 & $0 e$ & 47 & 2 & 26 & sar95 & 1.093 & 3275.72817 \\
\hline
\end{tabular}




\begin{tabular}{|c|c|c|c|c|c|c|c|c|c|c|c|c|c|c|c|c|c|c|}
\hline 0 & 0 & 1 & 2 & 0, & $20 f$ & 0 & 0 & 0 & 1 & 0 & 1 & $0 f$ & 61 & 2 & 37 & $\begin{array}{l}\text { sar95 } \\
\text { gir06 }\end{array}$ & 0.396 & 3892.84332 \\
\hline 0 & 0 & 1 & 2 & 0, & $20 f$ & 0 & 0 & 0 & 1 & 0 & 1 & $0 e$ & 18 & 2 & 19 & sar95 & 0.388 & 3892.84332 \\
\hline 0 & 0 & 1 & 2 & 0, & $20 f$ & 0 & 0 & 0 & 2 & 0 & 2 & $0 f$ & 47 & 2 & 26 & sar95 & 1.114 & 3275.73703 \\
\hline 0 & 0 & 1 & 2 & 0, & $00 e$ & 0 & 0 & 0 & 0 & 0 & 0 & $0 \mathrm{e}$ & 57 & 0 & 30 & lyu7b & 0.415 & 4508.01322 \\
\hline 0 & 0 & 1 & 2 & 0, & $00 e$ & 0 & 0 & 0 & 2 & 0 & 0 & $0 \mathrm{e}$ & 50 & 0 & 25 & sar95 & 1.774 & 3277.63071 \\
\hline 0 & 0 & 1 & 2 & 0 & $00 e$ & 0 & 0 & 0 & 1 & 0 & 1 & $0 e$ & 46 & 0 & 25 & $\begin{array}{l}\text { sar95 } \\
\text { gir06 }\end{array}$ & 0.435 & 3896.31944 \\
\hline 0 & 0 & 1 & 2 & 0, & $00 e$ & 0 & 0 & 0 & 1 & 0 & 1 & $0 f$ & 20 & 1 & 20 & sar95 & 0.307 & 3896.31944 \\
\hline 0 & 1 & 0 & 2 & 2, & $2-2 f$ & 0 & 0 & 0 & 1 & 1, & & $-1 f$ & 54 & 0 & 32 & $\begin{array}{l}\text { rin82 } \\
\text { lyu06 }\end{array}$ & 1.297 & 3259.22329 \\
\hline 0 & 1 & 0 & 2 & 2, & $20 f$ & 0 & 0 & 0 & 1 & 1 & 1 & $1 \mathrm{f}$ & 50 & 2 & 36 & $\begin{array}{l}\text { rin82 } \\
\text { lyu06 }\end{array}$ & 3.837 & 3260.47068 \\
\hline 0 & 1 & 0 & 2 & 2, & 20 e & 0 & 0 & 0 & 1 & 1 & 1 & $1 \mathrm{e}$ & 39 & 2 & 30 & $\begin{array}{l}\text { rin82 } \\
\text { lyu06 }\end{array}$ & 1.779 & 3260.52213 \\
\hline 0 & 0 & 1 & 1 & 1, & $1-1 e$ & 0 & 0 & 0 & 1 & 1, & & $-1 e$ & 50 & 0 & 25 & sar95 & 1.551 & 3281.21743 \\
\hline 0 & 0 & 1 & 1 & 1, & $1-1 e$ & 0 & 0 & 0 & 0 & 1 & 0 & $1 e$ & 42 & 0 & 26 & $\begin{array}{l}\text { sar95 } \\
\text { gir06 }\end{array}$ & 0.509 & 3880.13658 \\
\hline 0 & 0 & 1 & 1 & 1, & $1-1 e$ & 0 & 0 & 0 & 0 & 1, & 0 & $1 \mathrm{f}$ & 19 & 1 & 19 & sar95 & 0.316 & 3880.13658 \\
\hline 0 & 0 & 1 & 1 & 1, & $1-1 \mathrm{f}$ & 0 & 0 & 0 & 0 & 1 & 0 & $1 e$ & 20 & 1 & 20 & sar95 & 0.338 & 3888.77236 \\
\hline 0 & 0 & 1 & 1 & 1, & $1-1 f$ & 0 & 0 & 0 & 0 & 1, & 0 & $1 f$ & 39 & 0 & 25 & $\begin{array}{l}\text { sar95 } \\
\text { gir06 }\end{array}$ & 0.447 & 3888.77236 \\
\hline 0 & 0 & 1 & 1 & 1, & $1-1 \mathrm{f}$ & 0 & 0 & 0 & 1 & 1, & & $-1 f$ & 51 & 0 & 26 & sar95 & 1.952 & 3277.37569 \\
\hline 0 & 0 & 1 & 1 & 1, & $11 e$ & 0 & 0 & 0 & 1 & 1 & 1 & $1 e$ & 47 & 2 & 26 & sar95 & 0.708 & 3277.25526 \\
\hline 0 & 0 & 1 & 1 & 1, & $11 \mathrm{f}$ & 0 & 0 & 0 & 1 & 1 , & 1 & $1 \mathrm{f}$ & 47 & 2 & 26 & sar95 & 0.975 & 3277.25525 \\
\hline 0 & 0 & 1 & 1 & 1, & $11 \mathrm{e}$ & 0 & 0 & 0 & 0 & 1 & 0 & $1 e$ & 46 & 2 & 31 & $\begin{array}{l}\text { sar95 } \\
\text { gir06 }\end{array}$ & 0.402 & 3890.90327 \\
\hline 0 & 0 & 1 & 1 & 1, & $11 \mathrm{e}$ & 0 & 0 & 0 & 0 & 1, & 0 & $1 \mathrm{f}$ & 20 & 2 & 21 & sar95 & 0.417 & 3890.90327 \\
\hline 0 & 0 & 1 & 1 & 1, & $11 \mathrm{f}$ & 0 & 0 & 0 & 0 & 1, & 0 & $1 \mathrm{f}$ & 54 & 2 & 36 & $\begin{array}{l}\text { sar95 } \\
\text { gir06 }\end{array}$ & 0.530 & 3890.90327 \\
\hline 0 & 0 & 1 & 1 & 1, & $11 \mathrm{f}$ & 0 & 0 & 0 & 0 & 1, & 0 & $1 e$ & 20 & 2 & 21 & sar95 & 0.244 & 3890.90327 \\
\hline 1 & 0 & 0 & 1 & 1, & $1-1 e$ & 0 & 0 & 0 & 0 & 0 & 0 & $0 e$ & 53 & 0 & 30 & lyu $7 \mathrm{~b}$ & 0.338 & 4620.05743 \\
\hline 1 & 0 & 0 & 1 & 1, & $1-1 e$ & 0 & 0 & 0 & 1 & 0 & 1 & $0 e$ & 66 & 0 & 38 & $\begin{array}{l}\text { dcu } 91 \\
\text { gir06 } \\
\text { lyu07 }\end{array}$ & 1.098 & 4008.36365 \\
\hline 1 & 0 & 0 & 1 & 1, & $1-1 e$ & 0 & 0 & 0 & 1 & 0 & 1 & $0 f$ & 28 & 1 & 28 & $\begin{array}{l}\text { dcu } 91 \\
\text { lyu07 }\end{array}$ & 1.037 & 4008.36365 \\
\hline 1 & 0 & 0 & 1 & 1, & $11 e$ & 0 & 0 & 0 & 1 & 0 & 1 & $0 f$ & 24 & 2 & 25 & $\begin{array}{l}\text { dcu } 91 \\
\text { lyu07 }\end{array}$ & 0.543 & 4075.66942 \\
\hline 1 & 0 & 0 & 1 & 1, & $11 e$ & 0 & 0 & 0 & 1 & 0 & 1 & $0 e$ & 46 & 2 & 26 & $\begin{array}{l}\text { dcu } 91 \\
\text { lyu07 }\end{array}$ & 0.460 & 4075.66942 \\
\hline 1 & 0 & 0 & 1 & 1, & $11 f$ & 0 & 0 & 0 & 1 & 0 & 1 & $0 f$ & 61 & 2 & 36 & $\begin{array}{l}\text { dcu91 } \\
\text { gir06 } \\
\text { lyu07 }\end{array}$ & 0.517 & 4075.66946 \\
\hline 1 & 0 & 0 & 1 & 1, & $11 f$ & 0 & 0 & 0 & 1 & 0 & 1 & $0 e$ & 29 & 2 & 30 & $\begin{array}{l}\text { dcu } 91 \\
\text { lyu07 }\end{array}$ & 0.421 & 4075.66946 \\
\hline 1 & 0 & 0 & 1 & 1, & $1-1 f$ & 0 & 0 & 0 & 1 & 0 & 1 & $0 e$ & 26 & 1 & 26 & $\begin{array}{l}\text { dcu } 91 \\
\text { lyu07 }\end{array}$ & 0.317 & 4077.14564 \\
\hline 1 & 0 & 0 & 1 & 1, & $1-1 f$ & 0 & 0 & 0 & 1 & 0 & 1 & $0 f$ & 61 & 0 & 35 & $\begin{array}{l}\text { dcu } 91 \\
\text { lyu07 }\end{array}$ & 0.446 & 4077.14564 \\
\hline 0 & 1 & 0 & 1 & 3, & $1-1 e$ & 0 & 0 & 0 & 0 & 2 & 0 & $0 e$ & 26 & 0 & 21 & $\begin{array}{l}\text { rin82 } \\
\text { lyu06 }\end{array}$ & 1.785 & 3261.62186 \\
\hline 0 & 1 & 0 & 1 & $3,-$ & $-13 f$ & 0 & 0 & 0 & 0 & 2 & 0 & $2 f$ & 38 & 3 & 31 & $\begin{array}{l}\text { rin82 } \\
\text { lyu06 }\end{array}$ & 2.622 & 3262.82770 \\
\hline 0 & 1 & 0 & 1 & $3,-$ & $-13 e$ & 0 & 0 & 0 & 0 & 2 & 0 & $2 e$ & 36 & 3 & 26 & $\begin{array}{l}\text { rin82 } \\
\text { lyu06 }\end{array}$ & 3.606 & 3262.84803 \\
\hline 0 & 0 & 1 & 0 & 2, & $00 e$ & 0 & 0 & 0 & 0 & 2 & 0 & $0 \mathrm{e}$ & 45 & 0 & 24 & sar95 & 1.313 & 3277.93741 \\
\hline 0 & 0 & 1 & 0 & 2, & $00 e$ & 0 & 0 & 0 & 0 & 0 & 0 & $0 e$ & 52 & 0 & 26 & lyu $7 \mathrm{~b}$ & 0.117 & 4727.04969 \\
\hline 0 & 0 & 1 & 0 & 2, & $02 f$ & 0 & 0 & 0 & 0 & 2, & 0 & $2 f$ & 43 & 2 & 24 & sar95 & 1.397 & 3278.02121 \\
\hline 0 & 0 & 1 & 0 & 2, & $02 e$ & 0 & 0 & 0 & 0 & 2 & 0 & $2 e$ & 43 & 2 & 24 & sar95 & 1.377 & 3278.02133 \\
\hline 1 & 0 & 0 & 0 & 2, & $00 e$ & 0 & 0 & 0 & 0 & 1, & 0 & $1 e$ & 72 & 0 & 41 & $\begin{array}{l}\text { dcu91 } \\
\text { gir06 } \\
\text { lyu07 }\end{array}$ & 0.394 & 4070.98191 \\
\hline 1 & 0 & 0 & 0 & 2, & $00 e$ & 0 & 0 & 0 & 0 & 1, & 0 & $1 \mathrm{f}$ & 27 & 1 & 27 & $\begin{array}{l}\text { dcu } 91 \\
\text { lyu07 }\end{array}$ & 0.359 & 4070.98191 \\
\hline 1 & 0 & 0 & 0 & 2, & $02 f$ & 0 & 0 & 0 & 0 & 1, & 0 & $1 \mathrm{f}$ & 62 & 2 & 34 & $\begin{array}{l}\text { dcu91 } \\
\text { gir06 } \\
\text { lyu07 }\end{array}$ & 0.253 & 4080.35545 \\
\hline 1 & 0 & 0 & 0 & $2 ，$ & $02 \mathrm{f}$ & 0 & 0 & 0 & 0 & 1, & 0 & $1 e$ & 26 & 2 & 27 & $\begin{array}{l}\text { dcu91 } \\
\text { lyu07 }\end{array}$ & 0.661 & 4080.35545 \\
\hline 1 & 0 & 0 & 0 & 2, & $02 e$ & 0 & 0 & 0 & 0 & 1, & 0 & $1 \mathrm{f}$ & 29 & 2 & 30 & dcu91 & 1.375 & 4080.35549 \\
\hline
\end{tabular}




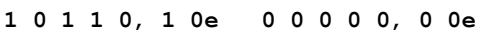
$\begin{array}{lllllllllllll}1 & 1 & 0 & 2 & 1,2-1 f * & 0 & 0 & 0 & 1 & 0, & 1 & 0 f\end{array}$ $\begin{array}{llllllllllll}1 & 1 & 0 & 2 & 1,2-1 e * & 0 & 0 & 0 & 1 & 0,1 & 0 e\end{array}$

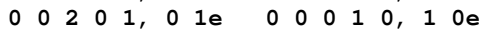

$\begin{array}{llllllllllllllllllllll}0 & 0 & 2 & 0 & 1, & 0 & 1 \mathrm{e} & 0 & 0 & 0 & 0 & 0, & 0 & 0 \mathrm{e}\end{array}$

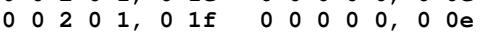
$\begin{array}{llllllllllllll}0 & 0 & 2 & 0 & 1, & 0 & 1 \mathrm{f} & 0 & 0 & 0 & 1 & 0, & 1 & 0 f\end{array}$

$\begin{array}{llllllllllllll}1 & 1 & 0 & 2 & 1 & 2-1 e^{*} & 0 & 0 & 0 & 0 & 0 & 0 & 0\end{array}$ $11021,2-1 e^{*} 00010,10 e$

$\begin{array}{lllllllllllll}1 & 1 & 0 & 2 & 1, & 2-1 \mathrm{f} * & 0 & 0 & 0 & 1 & 0, & 1 & \text { of }\end{array}$

$\begin{array}{lllllllllllll}1 & 1 & 0 & 2 & 1, & 2-1 \mathrm{f} * & 0 & 0 & 0 & 0 & 0, & 0 & 0 e\end{array}$ $\begin{array}{lllllllllllllllll}1 & 0 & 1 & 0 & 1, & 0 & 1 e & 0 & 0 & 0 & 0 & 1, & 0 & 1 e\end{array}$ $\begin{array}{llllllllllllll}1 & 0 & 1 & 0 & 1, & 0 & 1 \mathrm{f} & 0 & 0 & 0 & 0 & 1, & 0 & 1 \mathrm{f}\end{array}$

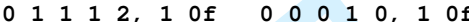
$\begin{array}{llllllllllllll}0 & 1 & 1 & 1 & 2, & 1 & 0 \mathrm{e} & 0 & 0 & 0 & 1 & 0, & 0 \mathrm{e}\end{array}$ $\begin{array}{lllllllllllll}0 & 1 & 1 & 1 & 2,-1 & 2 \mathrm{e} & 0 & 0 & 0 & 1 & 0, & 0 \mathrm{e}\end{array}$ $\begin{array}{lllllllllllll}0 & 1 & 1 & 1 & 2,-1 & 2 \mathrm{f} & 0 & 0 & 0 & 1 & 0, & 1 & 0 f\end{array}$ $\begin{array}{lllllllllllllll}2 & 0 & 0 & 1 & 0,1 & 0 \text { e } & 0 & 0 & 0 & 0 & 1, & 0 & 1 e\end{array}$ $\begin{array}{llllllllllllll}2 & 0 & 0 & 1 & 0, & 1 & 0 f & 0 & 0 & 0 & 0 & 1, & 0 & 1 f\end{array}$ $\begin{array}{llllllllllllll}1 & 1 & 0 & 1 & 2, & 1 & 0 \mathrm{f} & 0 & 0 & 0 & 0 & 1, & 0 & 1 \mathrm{f}\end{array}$ $\begin{array}{lllllllllllllllllllllll}1 & 1 & 0 & 1 & 2, & 0 \text { e } & 0 & 0 & 0 & 0 & 1, & 0 & 1 e\end{array}$ $\begin{array}{llllllllllllllllll}0 & 1 & 1 & 0 & 3, & 0 & 1 \mathrm{f} & 0 & 0 & 0 & 0 & 1, & 0 & 1 \mathrm{f}\end{array}$ $\begin{array}{lllllllllll}0 & 1 & 1 & 0 & 3, & 0 & 1 e\end{array}$ $2000001,001 \mathrm{f}$

$\begin{array}{lllllllllll}2 & 0 & 0 & 0 & 1, & 0 & 1 e\end{array}$ $\begin{array}{lllllll}0 & 0 & 0 & 0 & 1, & 0 & 1 e \\ 0 & 0 & 0 & 0 & 0, & 0 & 0 e\end{array}$ $\begin{array}{llllllll}0 & 0 & 0 & 0 & 0, & 0 & 0 e\end{array}$

$\begin{array}{llllllllllllll}2 & 0 & 0 & 0 & 1, & 0 & 1 f & 0 & 0 & 0 & 1 & 0, & 1 & \text { of }\end{array}$

$\begin{array}{lllllllllllllll}2 & 0 & 0 & 0 & 1, & 0 & 1 \mathrm{f} & 0 & 0 & 0 & 1 & 0, & 1 & 0 \mathrm{e}\end{array}$ $\begin{array}{lllllllllllllllll}2 & 0 & 0 & 0 & 1, & 0 & 1 e & 0 & 0 & 0 & 1 & 0, & 1 & 0 f\end{array}$ $\begin{array}{lllllllllllllllllllllll}2 & 0 & 0 & 0 & 1, & 0 & 1 \text { e } & 0 & 0 & 0 & 0 & 0, & 0 & 0 \text { e }\end{array}$ $\begin{array}{llllllllllllll}1 & 1 & 0 & 0 & 3, & 0 & 1 \mathrm{f} & 0 & 0 & 0 & 1 & 0, & 0 f\end{array}$ $\begin{array}{llllllllllllll}1 & 1 & 0 & 0 & 3, & 0 & 1 \mathrm{f} & 0 & 0 & 0 & 1 & 0, & 0 e\end{array}$ $\begin{array}{lllllllllllllll}1 & 1 & 0 & 0 & 3, & 0 & 1 e & 0 & 0 & 0 & 1 & 0, & 1 & 0 f\end{array}$

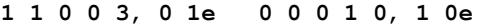
$\begin{array}{lllllllllllllllllllll}0 & 0 & 2 & 2 & 0, & 2 & 0 f & 0 & 0 & 0 & 0 & 1, & 0 & 1 f\end{array}$ $\begin{array}{llllllllllllll}0 & 0 & 2 & 2 & 0,2 & \text { of } & 0 & 0 & 0 & 0 & 1, & 0 & 1 e\end{array}$ $\begin{array}{llllllll}0 & 0 & 2 & 2 & 0, & 2 & \text { of }\end{array}$

$\begin{array}{llllllll}0 & 0 & 0 & 1 & 1, & 1 & 1 f\end{array}$

$\begin{array}{llllllll}0 & 0 & 2 & 2 & 0, & 2 & 0 e\end{array}$

$\begin{array}{llllllll}0 & 0 & 2 & 2 & 0, & 2 & 0 e\end{array}$

$\begin{array}{llllllll}0 & 0 & 2 & 2 & 0, & 0 & 0\end{array}$ $\begin{array}{lllllllllllll}0 & 0 & 2 & 2 & 0, & 0 & 0 e\end{array}$

$\begin{array}{lllllll}1 & 0 & 1 & 2 & 0, & 2 & 0 f\end{array}$

$\begin{array}{lllllllllll}1 & 0 & 1 & 2 & 0,2 & 0 e\end{array}$

$\begin{array}{lllllllllllll}1 & 0 & 1 & 2 & 0, & 0 & 0 e\end{array}$

$\begin{array}{llllllll}1 & 0 & 1 & 2 & 0, & 0 & 0\end{array}$

$\begin{array}{llllll}0 & 0 & 2 & 1 & 1,1-1 e\end{array}$

$\begin{array}{lllllll}0 & 0 & 0 & 0 & 1, & 0 & 1 \mathrm{e}\end{array}$ $\begin{array}{lllllll}0 & 0 & 0 & 1 & 1, & 1 & 1 e\end{array}$ $\begin{array}{llllllll}0 & 0 & 0 & 0 & 1, & 0 & 1 \mathrm{f}\end{array}$ $\begin{array}{lllllllllll}0 & 0 & 0 & 0 & 1, & 0 & 1 e\end{array}$ $\begin{array}{lllllllll}0 & 0 & 0 & 2 & 0, & 2 & \text { of }\end{array}$ $\begin{array}{llllllll}0 & 0 & 0 & 2 & 0, & 2 & 0 e\end{array}$ $\begin{array}{llllllllllllll}0 & 0 & 0 & 0 & 0, & 0 & 0 e\end{array}$ $\begin{array}{lllllllllllllll}0 & 0 & 0 & 2 & 0 & 0 & 0\end{array}$ $\begin{array}{lllllllllllllllll}0 & 0 & 0 & 0 & 2, & 0 & 0 e\end{array}$

$\begin{array}{llllll}1 & 0 & 1 & 1 & 1, & 1-1 \mathrm{e}\end{array}$

$\begin{array}{llllllllll}1 & 0 & 1 & 1 & 1, & 1 & 1 e\end{array}$

$\begin{array}{lllllll}1 & 0 & 1 & 1 & 1, & 1 & \text { f }\end{array}$

$\begin{array}{lllllll}1 & 0 & 1 & 1 & 1,1-1 f\end{array}$

$\begin{array}{llllllllllll}1 & 0 & 1 & 0 & 2, & 0 & 0 e\end{array}$

$\begin{array}{llllllll}1 & 0 & 1 & 0 & 2 & 0 & 2 \mathrm{f}\end{array}$

$\begin{array}{llllllll}1 & 0 & 1 & 0 & 2, & 0 & 2 \mathrm{e}\end{array}$

20001 , $1-1 \mathrm{e}$

$\begin{array}{lllllllll}2 & 0 & 0 & 1 & 1, & 1 & 1 e\end{array}$

$\begin{array}{llllllll}2 & 0 & 0 & 1 & 1, & 1 & 1\end{array}$

$200001,1,1$ ff

$\begin{array}{llllllll}2 & 0 & 0 & 1 & 1, & 1 & 1 f\end{array}$

$\begin{array}{llllllllllll}2 & 0 & 0 & 0 & 2, & 0 & 0 e\end{array}$

$\begin{array}{llllllll}2 & 0 & 0 & 0 & 2, & 0 & 2 \mathrm{f}\end{array}$

$\begin{array}{llllllll}2 & 0 & 0 & 0 & 2, & 0 & 2 f\end{array}$

$\begin{array}{llllllll}2 & 0 & 0 & 0 & 2, & 0 & 2 \mathrm{e}\end{array}$

$\begin{array}{llllllll}2 & 0 & 0 & 0 & 2, & 0 & 2 e\end{array}$

$\begin{array}{llllllll}0 & 0 & 0 & 1 & 1, & 1-1 e\end{array}$ $\begin{array}{llllllll}0 & 0 & 0 & 1 & 1, & 1 & 1 e\end{array}$ $\begin{array}{lllllll}0 & 0 & 0 & 1 & 1, & 1 & 1 \mathrm{f}\end{array}$ $\begin{array}{lllllll}0 & 0 & 0 & 1 & 1,1-1 f\end{array}$ $\begin{array}{lllllll}0 & 0 & 0 & 0 & 2, & 0 & 0 e\end{array}$ $\begin{array}{llllllll}0 & 0 & 0 & 0 & 2 & 0 & 2 f\end{array}$ $\begin{array}{llllllllllllllll}0 & 0 & 0 & 0 & 2, & 0 & 2 e\end{array}$ $\begin{array}{llllllllllllllllllll}0 & 0 & 0 & 2 & 0 & 0 & 0 \mathrm{e}\end{array}$ $\begin{array}{llllllll}0 & 0 & 0 & 2 & 0, & 2 & 0 f\end{array}$ $\begin{array}{llllllll}0 & 0 & 0 & 2 & 0,2 & 0\end{array}$ $\begin{array}{llllllll}0 & 0 & 0 & 2 & 0, & 2 & 0 \mathrm{e}\end{array}$ $\begin{array}{lllllll}0 & 0 & 0 & 2 & 0, & 2 & 0 f\end{array}$ $\begin{array}{llllllll}0 & 0 & 0 & 1 & 1,1-1 \mathrm{e}\end{array}$ $\begin{array}{llllllllll}0 & 0 & 0 & 1 & 1, & 1 & 1 e\end{array}$ $\begin{array}{lllllll}0 & 0 & 0 & 1 & 1, & 1 & 1 \mathrm{f}\end{array}$ $\begin{array}{lllllll}0 & 0 & 0 & 1 & 1, & 1 & 1 e\end{array}$ $\begin{array}{llllllll}0 & 0 & 0 & 1 & 1,1 & 1 & 1\end{array}$ $\begin{array}{lllllllllllllll}1 & 1 & 1 & 0 & 0 & 0 & 0 \mathrm{e}\end{array}$

$\begin{array}{llllllll}0 & 0 & 0 & 0 & 0 & 0 & 0 & 0 e\end{array}$

\begin{tabular}{|c|c|c|c|c|c|}
\hline 47 & 1 & 28 & vda02 & 0.422 & 7141.50299 \\
\hline 39 & 3 & 31 & kep96 & 0.437 & 6594.25916 \\
\hline 42 & 2 & 27 & kep96 & 0.502 & 6594.25916 \\
\hline 56 & 2 & 40 & $\begin{array}{l}\text { kep96 } \\
\text { rob08 }\end{array}$ & 0.529 & 6606.50586 \\
\hline 51 & 1 & 28 & vda02 & 0.385 & 7218.19964 \\
\hline 24 & 1 & 24 & vda02 & 0.289 & 7218.19965 \\
\hline 37 & 3 & 38 & $\begin{array}{l}\text { kep96 } \\
\text { rob08 }\end{array}$ & 0.400 & 6606.50587 \\
\hline 25 & 1 & 16 & vda02 & 0.607 & 7228.27124 \\
\hline 40 & 2 & 28 & $\begin{array}{l}\text { kep96 } \\
\text { rob08 }\end{array}$ & 0.562 & 6616.57746 \\
\hline 45 & 2 & 28 & $\begin{array}{l}\text { kep96 } \\
\text { rob08 }\end{array}$ & 0.496 & 6616.58056 \\
\hline 18 & 1 & 22 & vda02 & 0.397 & 7228.27434 \\
\hline 56 & 1 & 31 & kep96 & 0.359 & 6534.74916 \\
\hline 57 & 2 & 34 & kep96 & 0.339 & 6534.74916 \\
\hline 26 & 3 & 28 & rob08 & 1.413 & 6662.07802 \\
\hline 28 & 3 & 30 & rob08 & 1.459 & 6662.07968 \\
\hline 12 & 1 & 11 & rob08 & 3.264 & 6674.41487 \\
\hline 29 & 1 & 28 & rob08 & 1.566 & 6674.53735 \\
\hline 45 & 1 & 33 & kep96 & 0.393 & 6567.22885 \\
\hline 43 & 2 & 33 & kep96 & 0.309 & 6567.22886 \\
\hline 40 & 2 & 28 & kep96 & 0.511 & 6600.19072 \\
\hline 35 & 1 & 27 & kep96 & 0.553 & 6600.19073 \\
\hline 28 & 1 & 28 & rob08 & 1.822 & 6672.10942 \\
\hline 21 & 1 & 21 & rob08 & 1.166 & 6672.26918 \\
\hline 27 & 1 & 29 & vda02 & 0.299 & 7416.40041 \\
\hline 68 & 1 & 38 & $\begin{array}{l}\text { kep96 } \\
\text { rob08 }\end{array}$ & 0.313 & 6804.70663 \\
\hline 71 & 1 & 38 & $\begin{array}{l}\text { kep96 } \\
\text { rob08 }\end{array}$ & 0.522 & 6804.70663 \\
\hline 16 & 1 & 16 & rob08 & 1.307 & 6804.70663 \\
\hline 17 & 1 & 18 & rob08 & 0.979 & 6804.70663 \\
\hline 51 & 1 & 28 & vda02 & 0.312 & 7416.40041 \\
\hline 54 & 1 & 28 & rob08 & 0.900 & 6854.75932 \\
\hline 1 & 1 & 1 & rob08 & 3.345 & 6854.75932 \\
\hline 10 & 1 & 10 & rob08 & 1.369 & 6854.75935 \\
\hline 52 & 1 & 27 & rob08 & 1.437 & 6854.75935 \\
\hline 38 & 2 & 20 & rob08 & 1.319 & 6956.31575 \\
\hline 14 & 2 & 15 & rob08 & 1.495 & 6956.31575 \\
\hline 38 & 2 & 28 & $\begin{array}{l}\text { kep96 } \\
\text { rob08 }\end{array}$ & 0.577 & 6342.66773 \\
\hline 34 & 2 & 18 & rob08 & 2.594 & 6956.35447 \\
\hline 22 & 3 & 15 & rob08 & 0.911 & 6342.70646 \\
\hline 27 & 1 & 27 & rob08 & 1.763 & 6956.89393 \\
\hline 30 & 0 & 15 & rob08 & 1.363 & 6956.89393 \\
\hline 43 & 2 & 28 & kep96 & 0.496 & 6503.55145 \\
\hline 36 & 2 & 21 & kep96 & 0.487 & 6503.57532 \\
\hline 46 & 0 & 26 & vda02 & 0.417 & 7732.76330 \\
\hline 37 & 2 & 26 & kep96 & 0.443 & 6502.38079 \\
\hline 28 & 0 & 18 & $\begin{array}{l}\text { kep96 } \\
\text { rob08 }\end{array}$ & 0.561 & 6356.08851 \\
\hline 30 & 1 & 28 & kep96 & 0.382 & 6506.91254 \\
\hline 18 & 2 & 18 & kep96 & 0.442 & 6504.94704 \\
\hline 24 & 2 & 19 & kep96 & 0.646 & 6504.94707 \\
\hline 51 & 0 & 34 & kep96 & 0.565 & 6512.72508 \\
\hline 33 & 0 & 27 & kep96 & 0.490 & 6512.70665 \\
\hline 24 & 2 & 22 & kep96 & 0.627 & 6513.27206 \\
\hline 33 & 2 & 26 & kep96 & 0.578 & 6513.27204 \\
\hline 39 & 0 & 22 & rob08 & 2.577 & 6764.01188 \\
\hline 11 & 2 & 12 & rob08 & 0.793 & 6779.43585 \\
\hline 37 & 2 & 20 & rob08 & 0.741 & 6779.42766 \\
\hline 12 & 2 & 13 & rob08 & 0.695 & 6779.42771 \\
\hline 54 & 2 & 30 & rob08 & 0.840 & 6779.43590 \\
\hline 61 & 0 & 35 & rob08 & 1.563 & 6786.28982 \\
\hline 8 & 2 & 10 & rob08 & 1.333 & 6780.95951 \\
\hline 49 & 2 & 30 & rob08 & 1.616 & 6780.95950 \\
\hline 51 & 2 & 31 & rob08 & 1.219 & 6780.95954 \\
\hline 8 & 2 & 10 & rob08 & 1.098 & 6780.95953 \\
\hline 64 & 0 & 34 & vda02 & 0.325 & 8512.05644 \\
\hline
\end{tabular}




\section{Page 49 of 96}

Molecular Physics

1

2

3

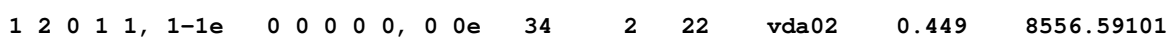

The vibrational states, $v^{\prime}$ and $v^{\prime \prime}$, are identified using the labels $v_{1} v_{2} v_{3} v_{4} v_{5}, l_{4} l_{5}, e / f$. Transitions are listed by order of increasing energy of the upper state. The vibrational assignments correspond to those of the dominant zero order state in the eigenvector composition at low $J$-values and may differ from some of the previous literature. The $\sigma$ values correspond to the standard deviation for each band in the global fit, in $10^{-3} \mathrm{~cm}^{-1}$. The $\Delta G_{c}$ value, in $\mathrm{cm}^{-1}$, is $\mathrm{G}_{\mathrm{c}}-\mathrm{G}^{\prime \prime}$.

ber98: Bermejo et al. ${ }^{21,22}$; ber99: Bermejo et al. ${ }^{22}$; dcu93: D'Cunha et al.$^{27}$; gir06: Girard et al. ${ }^{39}$; jac02: Jacquemart et al. ${ }^{27}$; jac03: Jacquemart et al. ${ }^{23}$; jac07 : Jacquemart et al..$^{34}$; kep96: Keppler et al. ${ }^{3}$; kou94: Kou et al. ${ }^{2} ;$ lyu06 : Lyulin et al..$^{33}$; lyu07 : Lyulin et al. ${ }^{35}$; lyu7b : Lyulin et al. ${ }^{36}$; lyu08 : Lyulin et al. ${ }^{37}$; man05: Mandin et al. ${ }^{32}$; pal72: Palmer et $a l .{ }^{25}$; rin82: Rinsland et al. ${ }^{26}$; rob07: Robert et al. ${ }^{24,}$ and refs listed therein ; rob08: present work ; sar95: Sarma et $a l .^{28}$; vda93: Vander Auwera et al. ${ }^{61}$; vda02: Vander Auwera et al. ${ }^{38}$ 
Table 3

\begin{tabular}{|c|c|c|c|c|c|c|c|c|c|c|c|c|c|c|c|c|c|c|}
\hline \multicolumn{6}{|c|}{$\boldsymbol{v}_{1} v_{2} v_{3} v_{4} v_{5}, l_{4} l_{5}, \mathrm{e} / \mathbf{f}$} & \multirow{2}{*}{$\frac{\boldsymbol{G} \boldsymbol{c}}{0.00000}$} & \multicolumn{4}{|c|}{$\boldsymbol{v}_{1} \boldsymbol{v}_{2} \boldsymbol{v}_{3} \boldsymbol{v}_{4} v_{5}, l_{4}$} & $l_{5}, \mathbf{e} / \mathbf{f}$ & \multirow{2}{*}{$\underbrace{\boldsymbol{G c}_{1}}_{3372.84725}$} & \multicolumn{4}{|c|}{$\boldsymbol{v}_{1} \boldsymbol{v}_{2} \boldsymbol{v}_{3} \boldsymbol{v}_{4} \boldsymbol{v}_{5}, \boldsymbol{l}_{4} \boldsymbol{l}_{5}, \mathrm{e} / \mathbf{f}$} & \multicolumn{2}{|c|}{ Gc } \\
\hline $\overrightarrow{0}$ & 0 & & 0 & 0, & $00 \mathrm{e}$ & & 1 & & 00 & 00 &, $00 \mathrm{e}$ & & 1 & 10 & 00 & 1, & $01 \mathrm{f}$ & 6052.07572 \\
\hline 0 & 0 & 0 & 1 & 0 & $10 e$ & 611.69378 & 0 & 1 & 02 & 21 & $2-1 e$ & 3881.23331 & 1 & 00 & 01 & 3 & $1-1 e$ & 6079.69072 \\
\hline 0 & 0 & 0 & 1 & 0, & $10 f$ & 611.69378 & 0 & 1 & 02 & 1 & $2-1 f$ & 3881.23331 & 0 & 20 & 00 & 3, & 0 if & 6093.51244 \\
\hline 0 & 0 & 0 & 0 & 1, & $01 \mathrm{f}$ & 729.15416 & 0 & 0 & 11 & 0 & $10 \mathrm{e}$ & 3897.16263 & 0 & 20 & 00 & 3 & $01 \mathrm{e}$ & 6093.51247 \\
\hline 0 & 0 & 0 & 0 & 1, & $01 \mathrm{e}$ & 729.15416 & 0 & 0 & 11 & 10 &, $10 f$ & 3897.16265 & & 20 & 03 & 1 , & $1-1 \mathrm{e}$ & 6413.90039 \\
\hline 0 & 0 & 0 & 2 & 0 & $20 f$ & 1228.80007 & 0 & 1 & 02 & 21 & $01 \mathrm{e}$ & 3908.13474 & 0 & 11 & 12 & 0 & $20 \mathrm{e}$ & 6447.78315 \\
\hline 0 & 0 & 0 & 2 & 0, & $20 \mathrm{e}$ & 1228.80826 & 0 & 2 & 00 & 0 & , $00 \mathrm{e}$ & 3933.89754 & 0 & 11 & 12 & 0 & $20 f$ & 6447.81394 \\
\hline 0 & 0 & 0 & 2 & 0 & $00 \mathrm{e}$ & 1230.38251 & 1 & 0 & 01 & 0 & $1,10 \mathrm{e}$ & 3968.88384 & 0 & 11 & 12 & 0 & $0 \mathrm{Oe}$ & 6449.09176 \\
\hline 0 & 0 & 0 & 1 & & $1-1 e$ & 1328.07331 & 1 & 0 & 01 & 10 &, $10 f$ & 3968.88384 & 0 & 02 & 20 & 0 , & $0 \mathrm{Oe}$ & 6502.34625 \\
\hline 0 & 0 & 0 & 1 & & $1-1 \mathrm{f}$ & 1340.55083 & 0 & 0 & 10 & $0 \quad 1$, & $01 \mathrm{e}$ & $4001.25976 *$ & 1 & 10 & 02 & 0 & $0 \mathrm{Oe}$ & 6513.99269 \\
\hline 0 & 0 & 0 & 1 & 1, & $11 \mathrm{e}$ & 1342.80217 & 0 & 0 & 10 & 1 & $01 \mathrm{f}$ & $4001.25977 *$ & 1 & 01 & 10 & 0, & $0 \mathrm{Oe}$ & 6556.46473 \\
\hline 0 & 0 & 0 & 1 & 1, & $11 \mathrm{f}$ & 1342.80218 & 0 & 0 & 10 & $0 \begin{array}{ll}0 & 1\end{array}$ &, $01 f$ & $4015.53618 *$ & 1 & 10 & 01 & 1, & $1-1 \mathrm{e}$ & 6623.13650 \\
\hline 0 & 0 & 0 & 0 & 2 & $00 e$ & 1449.11228 & 0 & 0 & 10 & $0 \quad 1$ &, $01 \mathrm{e}$ & $4015.53619 *$ & 0 & 20 & 01 & 3 & $1-1 \mathrm{e}$ & 6654.25190 \\
\hline 0 & 0 & 0 & 0 & 2 & $02 \mathrm{f}$ & 1458.29367 & 1 & 0 & 00 & $0 \quad 1$, & $01 \mathrm{e}$ & 4091.17259 & 0 & 11 & 10 & 2 & $00 \mathrm{e}$ & 6690.42425 \\
\hline 0 & 0 & 0 & 0 & 2 & $02 e$ & 1458.29370 & 1 & 0 & 00 & 1 & $01 \mathrm{f}$ & 4091.17259 & 2 & 00 & 00 & 0 & $0 \mathrm{Oe}$ & 6709.02113 \\
\hline 0 & 0 & 0 & 3 & 0 & $30 e$ & 1851.35850 & 0 & 1 & 00 & 03 &, $01 \mathrm{f}$ & 4138.87768 & 1 & 10 & 00 & 2 & $00 \mathrm{e}$ & 6759.23936 \\
\hline 0 & 0 & 0 & 3 & 0 & 3 of & 1851.37430 & 0 & 1 & 00 & $0 \quad 3$ & $01 \mathrm{e}$ & 4138.87771 & 0 & 02 & 21 & 0 & 1 of & 7091.28119 \\
\hline 0 & 0 & 0 & 3 & 0 , & $10 f$ & 1854.59535 & 0 & 1 & 03 & 1 & $3-1 f$ & 4486.11686 & 0 & 02 & 21 & 0, & $10 e$ & 7091.28120 \\
\hline 0 & 0 & 0 & 3 & 0 & $10 \mathrm{e}$ & 1854.60829 & 0 & 1 & 03 & 1 & $3-1 e$ & 4486.11992 & 1 & 01 & 11 & 0 & $10 \mathrm{f}$ & 7141.50298 \\
\hline 0 & 0 & 0 & 2 & & $2-1 e$ & 1939.99999 & 0 & 1 & 03 & 1 & $1-1 e$ & 4488.83815 & 1 & 01 & 11 & 0, & $10 \mathrm{e}$ & 7141.50299 \\
\hline 0 & 0 & 0 & 2 & 1, & $2-1 f$ & 1940.00000 & 0 & 0 & 12 & 0 &, $20 \mathrm{e}$ & 4504.53643 & 1 & 10 & 02 & 1 & $2-1 e$ & 7205.95294 * \\
\hline 0 & 0 & 0 & 2 & 1, & $01 \mathrm{e}$ & 1959.69158 & 0 & 0 & 12 & 20 &, $20 f$ & 4504.53710 & 1 & 10 & 02 & 1, & $2-1 f$ & 7205.95294 * \\
\hline 0 & 0 & 0 & 2 & 1, & $01 \mathrm{f}$ & 1959.69197 & 0 & 0 & 12 & 20 & $1,00 \mathrm{e}$ & 4508.01322 & 0 & 02 & 20 & 1, & $01 \mathrm{e}$ & 7218.19964 \\
\hline 0 & 0 & 0 & 2 & 1, & 2 if & 1961.93212 & 0 & 1 & 02 & 22 & $2-2 f$ & 4599.77412 & 0 & 02 & 20 & 1, & $01 \mathrm{f}$ & 7218.19965 \\
\hline 0 & 0 & 0 & 2 & 1, & $21 \mathrm{e}$ & 1961.93252 & 0 & 1 & 02 & $\begin{array}{ll}2 & 2 \\
2\end{array}$ & 2 of & 4603.27286 & 1 & 10 & 02 & 1, & $2-1 e$ & 7228.27124 * \\
\hline 0 & 1 & 0 & 0 & 0 & $00 \mathrm{e}$ & 1974.31602 & 0 & 1 & 02 & 22 & $20 \mathrm{e}$ & 4603.32430 & 1 & 10 & 02 & 1, & $2-1 f$ & $7228.27434^{*}$ \\
\hline 0 & 0 & 0 & 1 & 2, & $10 f$ & 2047.87622 & 0 & 0 & 11 & 11 & $1-1 \mathrm{e}$ & 4609.29074 & 1 & 01 & 10 & 1 , & $01 \mathrm{f}$ & 7263.90332 \\
\hline 0 & 0 & 0 & 1 & 2 & $10 \mathrm{e}$ & 2047.87622 & 0 & 0 & 11 & 11 & $1-1 f$ & 4617.92652 & 1 & 01 & 10 & 1 , & $01 \mathrm{e}$ & 7263.90332 \\
\hline 0 & 0 & 0 & 1 & $2,-$ & $-12 \mathrm{e}$ & 2065.79297 & 0 & 0 & 11 & 11 & $11 \mathrm{e}$ & 4620.05743 & 0 & 11 & 11 & 2, & $10 f$ & 7273.77180 \\
\hline 0 & 0 & 0 & 1 & $2,-$ & $-12 f$ & 2065.79298 & 0 & 0 & 11 & 11 &, $11 f$ & 4620.05743 & 0 & 11 & 11 & 2, & $10 e$ & 7273.77346 \\
\hline 0 & 0 & 0 & 1 & 2 & $12 \mathrm{e}$ & 2074.18018 & 1 & 0 & 01 & 11 &, $1-1 \mathrm{e}$ & 4673.63162 & 0 & 11 & 11 & $2,-$ & $-12 e$ & 7286.10865 \\
\hline 0 & 0 & 0 & 1 & 2 & $12 f$ & 2074.18024 & 1 & 0 & 01 & 11 & $11 \mathrm{e}$ & 4687.36320 & 0 & 11 & 11 & $2,-$ & $-12 f$ & 7286.23113 \\
\hline 0 & 0 & 0 & 0 & & $01 \mathrm{f}$ & 2169.15707 & 1 & 0 & 01 & $\begin{array}{lll}1 & 1\end{array}$ & 1 if & 4687.36324 & 2 & 00 & $\begin{array}{ll}0 & 1\end{array}$ & 0, & $10 \mathrm{e}$ & 7296.38301 \\
\hline 0 & 0 & 0 & 0 & 3, & $01 \mathrm{e}$ & 2169.15709 & 1 & 0 & 01 & & $1-1 \mathrm{f}$ & 4688.83942 & 2 & 00 & 01 & 0, & 1 of & 7296.38302 \\
\hline 0 & 0 & 0 & 0 & 3, & $03 e$ & 2187.45100 & 0 & 1 & 01 & 3 &, $1-1 \mathrm{e}$ & 4710.73414 & 1 & 10 & 01 & 2 & 1 of & 7329.34488 \\
\hline 0 & 0 & 0 & 0 & 3, & $03 f$ & 2187.45104 & 0 & 1 & 01 & 3 & $-13 f$ & 4721.12137 & 1 & 10 & 01 & 2, & $10 \mathrm{e}$ & 7329.34489 \\
\hline 0 & 0 & 0 & 3 & 1, & $3-1 f$ & 2556.79484 & 0 & 1 & 01 & & $-13 e$ & 4721.14173 & 0 & 1 & 10 & 3 & $01 \mathrm{f}$ & 7401.26358 \\
\hline 0 & 0 & 0 & 3 & & $3-1 e$ & 2556.79505 & 0 & 0 & 10 & 2 & 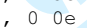 & 4727.04969 & 0 & 11 & 10 & 3 & $01 \mathrm{e}$ & 7401.42334 \\
\hline 0 & 0 & 0 & 3 & 1 , & $1-1 \mathrm{e}$ & 2560.59503 & 0 & 0 & 10 & $\begin{array}{ll}0 & 2 \\
0\end{array}$ & $02 f$ & 4736.31488 & 2 & 00 & $\begin{array}{ll}0 & 0\end{array}$ & 1 , & $01 \mathrm{e}$ & 7416.40041 \\
\hline 0 & 1 & 0 & 1 & 0, & $10 \mathrm{e}$ & 2573.53545 & 0 & 0 & 10 & 2 & $\therefore \quad 02 \mathrm{e}$ & 4736.31503 & 2 & 00 & 00 & 1 , & $01 \mathrm{f}$ & 7416.40041 \\
\hline 0 & 1 & 0 & 1 & 0 & 1 of & 2573.53545 & 1 & 0 & 00 & 2 & $\therefore \quad 0 \mathrm{e}$ & 4800.13607 & 1 & 10 & 00 & 3 & $01 \mathrm{f}$ & 7466.45310 \\
\hline 0 & 0 & 0 & 3 & 1, & $1-1 \mathrm{f}$ & 2583.86715 & 1 & 0 & 00 & 02 & $02 \mathrm{f}$ & 4809.50961 & 1 & 10 & 00 & 3 & $01 \mathrm{e}$ & 7466.45313 \\
\hline 0 & 0 & 0 & 3 & 1, & $11 \mathrm{f}$ & 2584.94806 & 1 & 0 & 00 & 02 & $\therefore \quad 2 e$ & 4809.50965 & 0 & 02 & 22 & 0, & $20 f$ & 7685.46991 \\
\hline 0 & 0 & 0 & 3 & 1, & $11 \mathrm{e}$ & 2584.96025 & 0 & 0 & 13 & 30 & $10 \mathrm{e}$ & $5101.54938 *$ & 0 & 02 & 22 & 0, & $20 \mathrm{e}$ & 7685.50863 \\
\hline 0 & 0 & 0 & 2 & 2, & $2-2 e$ & 2648.01406 & 0 & 0 & 13 & 30 & 1 , 1 f & $5101.62936 *$ & 0 & 02 & 22 & 0 & $0 \mathrm{Oe}$ & 7686.04809 \\
\hline 0 & 0 & 0 & 2 & 2, & $2-2 f$ & 2661.19038 & 0 & 0 & 13 & 0 & 1 of & $5123.39551 *$ & 1 & 01 & 12 & 0 & $20 f$ & 7732.35152 \\
\hline 0 & 0 & & 2 & 2, & $20 f$ & 2661.42794 & 0 & 0 & 13 & 30 & $10 \mathrm{e}$ & $5123.39959 *$ & 1 & 01 & 12 & 0 & $20 \mathrm{e}$ & 7732.38358 \\
\hline 0 & 0 & 0 & 2 & 2 & $20 e$ & 2661.42927 & 0 & 2 & 01 & 11 &, $1-1 \mathrm{e}$ & 5230.22853 & 1 & 01 & 12 & 0, & $0 \mathrm{Oe}$ & 7732.76330 \\
\hline 0 & 1 & 0 & 0 & 1, & $01 \mathrm{e}$ & 2701.90945 & 0 & 1 & 10 & 00 & $1,00 \mathrm{e}$ & 5260.02212 & 0 & 02 & 21 & 1, & $1-1 \mathrm{e}$ & 7805.20079 \\
\hline 0 & 1 & 0 & 0 & 1, & $01 \mathrm{f}$ & 2701.90945 & 1 & 0 & 02 & 21 & $2-1 e$ & $5268.54595 *$ & 1 & 01 & 11 & 1, & $1-1 \mathrm{e}$ & 7834.98585 \\
\hline 0 & 0 & 0 & 1 & & $1-1 e$ & 2757.79692 & 1 & 0 & 02 & 2 & $2-1 f$ & $5268.54595 *$ & 1 & 01 & 11 & 1, & $11 \mathrm{e}$ & 7847.74921 \\
\hline 0 & 0 & 0 & 1 & 3, & $11 \mathrm{f}$ & 2768.45032 & 1 & 0 & 02 & 21 & $2-1 e$ & 5289.09956 * & 1 & 01 & 11 & 1, & $11 \mathrm{f}$ & 7847.74925 \\
\hline 0 & 0 & 0 & 1 & 3, & $11 \mathrm{e}$ & 2768.45123 & 1 & 0 & 02 & 2 & $2-1 f$ & $5289.10486 *$ & 1 & 01 & 11 & 1, & $1-1 \mathrm{f}$ & 7853.27591 \\
\hline 0 & 0 & 0 & 1 & & $1-1 \mathrm{f}$ & 2783.65003 & 1 & 0 & 02 & 2 &, $01 \mathrm{e}$ & 5301.13135 & 1 & 01 & 10 & 2 & $0 \mathrm{Oe}$ & 7961.81893 \\
\hline 0 & 0 & 0 & 1 & $3,-$ & $-13 e$ & 2790.76333 & 1 & 0 & 02 & 21 & $01 f$ & 5301.13155 & 1 & 01 & 10 & 2 & $02 f$ & 7971.56573 \\
\hline 0 & 0 & 0 & 1 & $3,-$ & $-13 f$ & 2790.76350 & 1 & 1 & 00 & 00 &, $00 e$ & 5335.54888 & 1 & 01 & 10 & 2 & $02 e$ & 7971.56574 \\
\hline 0 & 0 & 0 & 0 & & $00 \mathrm{e}$ & 2880.21891 & 1 & 0 & 01 & 12 & 1 of & 5381.56126 & 2 & 00 & 01 & 1, & $1-1 \mathrm{e}$ & 7994.39439 \\
\hline 0 & 0 & 0 & 0 & 4, & $02 \mathrm{f}$ & 2889.32383 & 1 & 0 & 01 & 12 & $10 \mathrm{e}$ & 5381.56127 & 2 & 00 & 01 & 1, & $1 \mathrm{le}$ & 8008.23592 \\
\hline 0 & 0 & 0 & 0 & & $02 e$ & 2889.32524 & 1 & 0 & 00 & 0 &, $01 \mathrm{f}$ & 5509.22211 & 2 & 00 & 01 & 1, & $11 \mathrm{f}$ & 8008.23597 \\
\hline 0 & 0 & 1 & 0 & & $00 \mathrm{e}$ & 3281.89920 & 1 & 0 & 00 & 03 & $01 \mathrm{e}$ & 5509.22214 & 2 & 00 & 00 & 2 & $0 \mathrm{Oe}$ & 8114.36313 \\
\hline 0 & 1 & 0 & 1 & & $1-1 e$ & 3294.83960 & 0 & 2 & 02 & 21 & $2-1 e$ & 5816.86353 & 2 & 00 & 00 & 2 & $02 \mathrm{f}$ & 8123.76168 \\
\hline 0 & 1 & 0 & 1 & & $1-1 \mathrm{f}$ & 3300.63499 & 0 & 2 & 02 & 2 & $2-1 f$ & 5816.86354 & 2 & 00 & 00 & 2 & $02 \mathrm{e}$ & 8123.76171 \\
\hline 0 & 1 & 0 & 1 & & $11 \mathrm{f}$ & 3303.01742 & 0 & 1 & 11 & 1 & 1 of & 5850.64984 & 1 & 11 & 10 & 0 & $00 \mathrm{e}$ & 8512.05644 \\
\hline 0 & 1 & 0 & 1 & & $11 \mathrm{e}$ & 3303.01745 & 0 & 1 & 1 & 1 &, $10 \mathrm{e}$ & 5850.67614 & 1 & 20 & 01 & 1 & $1-1 \mathrm{e}$ & 8556.59101 \\
\hline & & & & & & & 1 & & & &, $01 \mathrm{e}$ & 6052.07572 & & & & & & \\
\hline
\end{tabular}

* These states have the same vibrational label because they share the dominant contribution in their eigenvector composition.

The vibrational assignments correspond to those of the dominant zero order state in the eigenvector composition at low J-values and may differ from some of the previous literature. 
Table 4

$E_{v r}=G(v, l)+B_{v}\left[J(J+1)-k^{2}\right]-D_{v}\left[J(J+1)-k^{2}\right]^{2}+H_{v}\left[J(J+1)-k^{2}\right]^{3}$

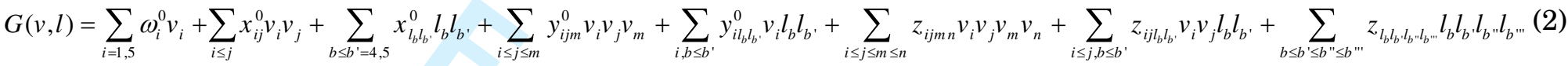

$B_{v}=B_{0}-\sum_{i} \alpha_{i}^{0} v_{i}+\sum_{i \leq j} \gamma_{i j}^{0} v_{i} v_{j}+\sum_{b \leq b^{\prime}} \gamma_{l_{b} l^{\prime}} l_{b} l_{b^{\prime}}+\sum_{i \leq j \leq m} \varepsilon_{i j m} v_{i} v_{j} v_{m}+\sum_{i, b \leq b} \varepsilon_{i l_{b} b^{\prime}} v_{i} l_{b} l_{b}$

$D_{v}=D_{0}+\sum_{i} \beta_{i}^{0} v_{i}+\sum_{t \leq t} \beta_{l_{l} l^{\prime}} l_{t} l_{t^{\prime}}$

(4)

$H_{v}=H_{0}$

$\left\langle l_{4}, l_{5}|\tilde{H}| l_{4} \mp 2, l_{5} \pm 2\right\rangle=1 / 4 r_{45}\left[\left(v_{4} \pm l_{4}\right)\left(v_{4} \mp l_{4}+2\right)\left(v_{5} \mp l_{5}\right)\left(v_{5} \pm l_{5}+2\right)\right]^{1 / 2}$

$r_{45}=r_{45}^{0}+\sum_{i} r_{45 i}\left(v_{i}-\delta_{i 4}-\delta_{i 5}\right)+\sum_{i \leq j} r_{45 i j}\left(v_{i}-\delta_{i 4}-\delta_{i 5}\right)\left(v_{j}-\delta_{j 4}-\delta_{j 5}\right)+\sum_{b \leq b^{\prime}} r_{45 l_{b} b^{\prime},}\left(l_{b} \mp 1\right)\left(l_{b^{\prime}} \pm 1\right)+\left[r_{45 J}^{0}+\sum_{i} r_{45 J i}\left(v_{i}-\delta_{i 4}-\delta_{i 5}\right)+r_{45 J J} J(J+1)\right] J(J+1)$

$\left\langle l_{b}|\tilde{H}| l_{b} \pm 2\right\rangle=1 / 4 q_{b}\left[\left(v_{b} \mp l_{b}\right)\left(v_{b} \pm l_{b}+2\right)\right]^{1 / 2} F_{ \pm}(J, k) F_{ \pm}(J, k \pm 1)$

$q_{b}=q_{b}^{0}+\sum_{i} q_{b i}\left(v_{i}-\delta_{i b}\right)+\sum_{i \leq j} q_{b i j}\left(v_{i}-\delta_{i b}\right)\left(v_{j}-\delta_{j b}\right)+\left[q_{b J}^{0}+\sum_{i} q_{b J i}\left(v_{i}-\delta_{i b}\right)\right](J(J+1)$

$\left\langle l_{b}|\tilde{H}| l_{b} \pm 4\right\rangle=1 / 4 u_{b b}\left[\left(v_{b} \mp l_{b}\right)\left(v_{b} \pm l_{b}+2\right)\left(v_{b} \mp l_{b}-2\right)\left(v_{b} \pm l_{b}+4\right)\right]^{1 / 2} F_{ \pm}(J, k) F_{ \pm}(J, k \pm 1) F_{ \pm}(J, k \pm 2) F_{ \pm}(J, k \pm 3)$

$\left\langle l_{b} l_{b^{\prime}}|\tilde{H}| l_{b} \pm 2, l_{b^{\prime}} \pm 2\right\rangle=1 / 4 u_{b b^{\prime}}\left[\left(v_{b} \mp l_{b}\right)\left(v_{b} \pm l_{b}+2\right)\left(v_{b^{\prime}} \mp l_{b^{\prime}}\right)\left(v_{b^{\prime}} \pm l_{b^{\prime}}+2\right)\right]^{1 / 2} F_{ \pm}(J, k) F_{ \pm}(J, k \pm 1) F_{ \pm}(J, k \pm 2) F_{ \pm}(J, k \pm 3)$

$F_{ \pm}(J, k)=[J(J+1)-k(k \pm 1)]^{1 / 2}$

$\left\langle v_{2}, v_{3}, v_{4}^{l_{4}}, v_{5}^{l_{5}}|\tilde{H}| v_{2}-1, v_{3}+1,\left(v_{4}-1\right)^{l_{4} \pm 1},\left(v_{5}-1\right)^{l_{5} \mp 1}\right\rangle=-1 / 8 K_{3 / 245}\left[v_{2}\left(v_{3}+1\right)\left(v_{4} \mp l_{4}\right)\left(v_{5} \pm l_{5}\right)\right]^{1 / 2}$ 


$$
K_{3 / 245}=K_{3 / 245}^{0}+\sum_{i} K_{3 / 245, i}\left(v_{i}-\delta_{i 2}-\delta_{i 4}-\delta_{i 5}\right)+K_{3 / 245, J}\left[J(J+1)-k^{2}\right]+K_{3 / 245, J J}\left[J(J+1)-k^{2}\right]^{2}
$$

$$
O_{3 / 245}=O_{3 / 245}^{0}+\sum_{i} O_{3 / 245, i}\left(v_{i}-\delta_{i 2}-\delta_{i 4}-\delta_{i 5}\right)+O_{3 / 245, J}\left[J(J+1)-k^{2}\right]
$$

$\left\langle v_{4}^{l_{4}}, v_{5}^{l_{5}}|\tilde{H}|\left(v_{4}+2\right)^{l_{4}},\left(v_{5}-2\right)^{l_{5}}\right\rangle=1 / 4 K_{44 / 55}\left[\left(v_{4}-l_{4}+2\right)\left(v_{4}+l_{4}+2\right)\left(v_{5}+l_{5}\right)\left(v_{5}-l_{5}\right)\right]^{1 / 2}$

$\left\langle v_{4}^{l_{4}}, v_{5}^{l_{5}}|\tilde{H}|\left(v_{4}+2\right)^{l_{4} \pm 2},\left(v_{5}-2\right)^{l_{5} \mp 2}\right\rangle=1 / 8 K_{44 / 55}^{\prime}\left[\left(v_{4} \pm l_{4}+2\right)\left(v_{4} \pm l_{4}+4\right)\left(v_{5} \pm l_{5}\right)\left(v_{5} \pm l_{5}-2\right)\right]^{1 / 2}$

$\left\langle v_{4}^{l_{4}}, v_{5}^{l_{5}}|\tilde{H}|\left(v_{4}+2\right)^{l_{4}},\left(v_{5}-2\right)^{l_{5} \pm 2}\right\rangle=-1 / 4 O_{44 / 55}\left[\left(v_{4}-l_{4}+2\right)\left(v_{4}+l_{4}+2\right)\left(v_{5} \mp l_{5}\right)\left(v_{5} \mp l_{5}-2\right)\right]^{1 / 2} F_{ \pm}(J, k) F_{ \pm}(J, k \pm 1)$

a In the terms, $i, j, m, n$ refer to the five modes of vibration, $b$ and $b$ ' specifically to the bending ones, and $k=l_{4}+l_{5}$. The notation $\delta_{i j}$ is the usual Kronecker symbol. 
2 Table 5 (each column is to be read until the end of next page)

4
5
6
7
8
9
10
11
12
13
14
15
16
17
18
19
20
21
22
23
24
25
26
27
28
29
30
31
32
33
34
35
36
37
38
39
40
41
42
43
44
45
46
47
48
49
50
51
52
53
54
55
56
57
58
59
60

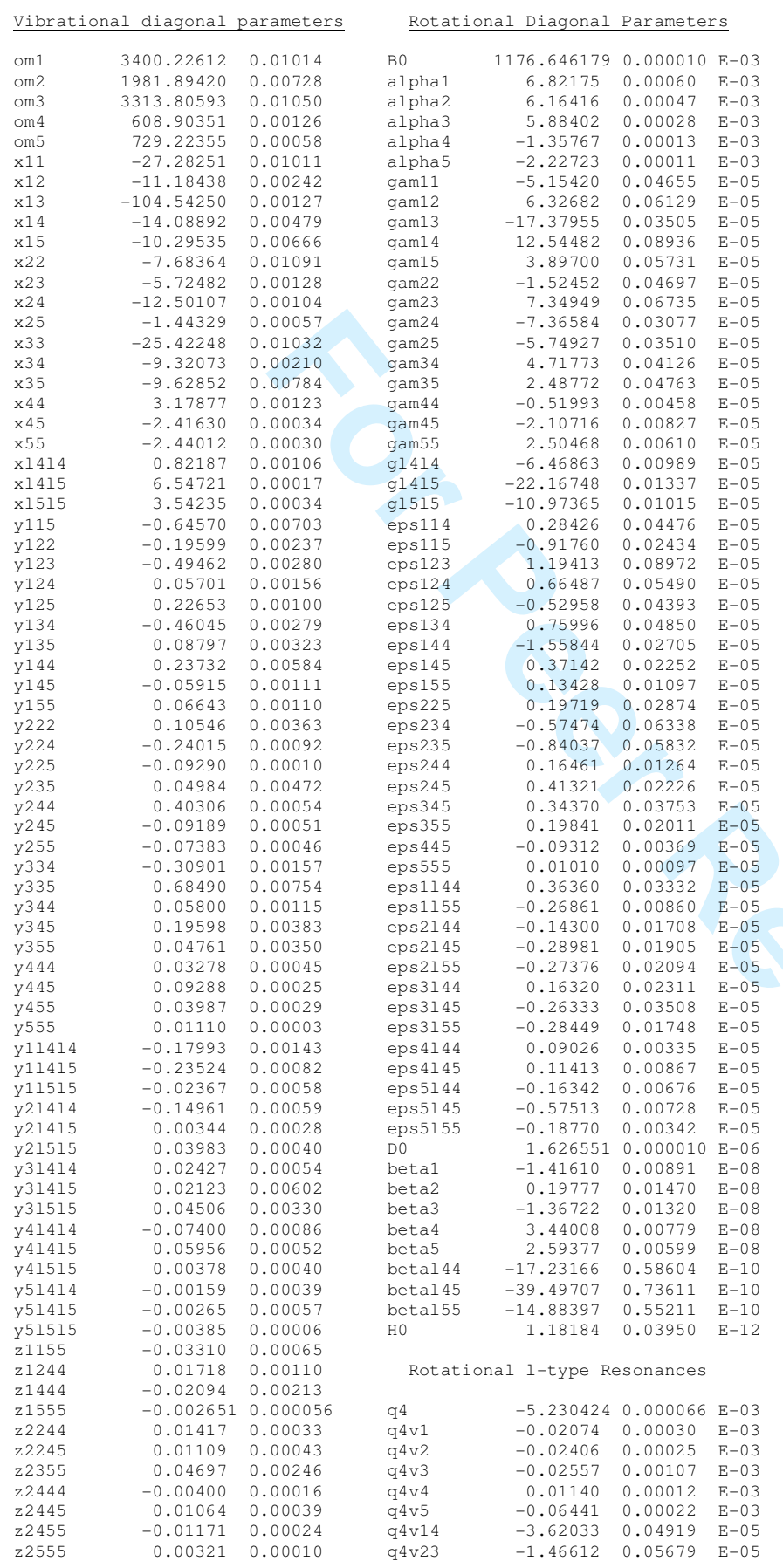

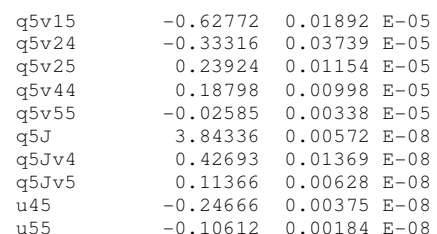

$\begin{array}{lll}\mathrm{k} 1534 \mathrm{v} 3 & -4.98501 & 0.04034\end{array}$ 


$\begin{array}{lcl}\text { z3344 } & -0.01993 & 0.00046 \\ \text { z3444 } & -0.00503 & 0.00031 \\ \text { z3455 } & -0.08792 & 0.00316 \\ \text { z3555 } & -0.03107 & 0.00111 \\ \text { z4444 } & -0.003576 & 0.000060 \\ \text { z4445 } & -0.002867 & 0.000015 \\ \text { z4455 } & -0.00566 & 0.00015 \\ \text { z121515 } & -0.02480 & 0.00043 \\ \text { z221414 } & -0.00570 & 0.00031 \\ \text { z241414 } & 0.00269 & 0.00022 \\ \text { z341414 } & -0.02841 & 0.00026 \\ \text { z441414 } & 0.00938 & 0.00020 \\ \text { z451414 } & 0.002008 & 0.000074 \\ \text { z14444 } & 0.002975 & 0.000068 \\ \text { z14555 } & 0.000605 & 0.000031 \\ \text { z15555 } & -0.000081 & 0.000002\end{array}$
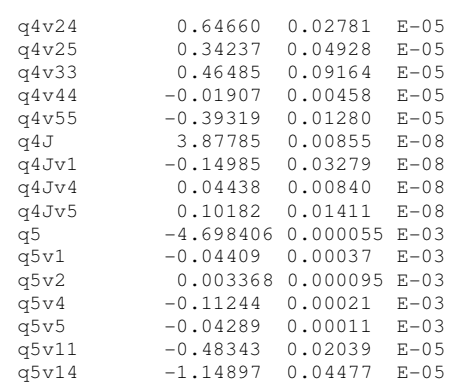

$\begin{array}{lrll}\text { k1534v4 } & 0.08935 & 0.01338 & \\ \text { k1534v5 } & -0.78033 & 0.02186 & \\ \text { k1534J } & -37.16915 & 0.71311 & \mathrm{E}-05 \\ 01534 & -9.82520 & 0.09458 & \mathrm{E}-05 \\ \text { k4455 } & -8.57758 & 0.00378 & \\ \text { k4455v1 } & -1.27134 & 0.02881 & \\ \text { k4455v2 } & -0.35485 & 0.00707 & \\ \text { k4455v3 } & -1.46397 & 0.00975 & \\ \text { k4455v5 } & 0.09946 & 0.00130 & \\ \text { k4455J } & 18.01478 & 0.13423 & \mathrm{E}-05 \\ \text { kp4455 } & 4.88246 & 0.02540 & \\ \text { kp4455v1 } & -0.29289 & 0.01767 & \\ \text { kp4455v3 } & 0.50071 & 0.01716 & \\ \text { kp4455v5 } & -0.03025 & 0.00379 & \\ \text { kp4455J } & -6.80235 & 0.19095 & \mathrm{E}-05 \\ 04455 & 3.92494 & 0.09334 & \mathrm{E}-05\end{array}$

The parameters are labeled in agreement with the model defined in the text. They are listed as printed by the program. 


\section{Page 55 of 96}

1

2

3

4

5

6

7

8

9

10

11

12

13

14

15

16

17

18

19

20

21

22

23

24

25

26

27

28

29

30

31

32

33

34

35

36

37

38

39

40

41

42

43

44

45

46

47

48

49

50

51

52

53

54

55

56

57

58

59

60

Table 6

\begin{tabular}{|l|c|c|c|c|c|}
\hline Accuracy & $\leq 4 \times 10^{-4} \mathrm{~cm}^{-1}$ & $\leq 9 \times 10^{-4} \mathrm{~cm}^{-1}$ & $\leq 2 \times 10^{-3} \mathrm{~cm}^{-1}$ & $\leq 10^{-2} \mathrm{~cm}^{-1}$ & Total \\
\hline Lines fitted & 6513 & 1699 & 1525 & 1013 & 10750 \\
\hline Fraction & $60.59 \%$ & $15.80 \%$ & $14.19 \%$ & $9.42 \%$ & $100 \%$ \\
\hline
\end{tabular}


Table 7

\begin{tabular}{|c|c|c|c|c|c|}
\hline & ${ }^{12} \mathrm{C}_{2} \mathrm{H}_{2}$ & ${ }^{13} \mathrm{CH}^{12} \mathrm{CH}$ & & ${ }^{12} \mathrm{C}_{2} \mathrm{H}_{2}$ & ${ }^{13} \mathrm{CH}^{12} \mathrm{CH}$ \\
\hline $\mathrm{x} 14$ & -14.09 & -13.50 & Alpha1 (E-03) & 6.82 & 6.52 \\
\hline $\mathrm{x} 15$ & -10.29 & -10.88 & Alpha2 (E-03) & 6.16 & 5.98 \\
\hline$x 22$ & -7.68 & -7.15 & Alpha3 (E-03) & 5.88 & 5.55 \\
\hline$x 23$ & -5.72 & -5.21 & Alpha4 (E-03) & -1.36 & -1.19 \\
\hline$x 24$ & -12.50 & -12.45 & Alpha5 (E-03) & -2.23 & -2.07 \\
\hline$x 25$ & -1.44 & -1.54 & K1244 & 11.85 & 10.92 \\
\hline$x 33$ & -25.42 & -26.99 & K1255 & 7.83 & 7.00 \\
\hline x34 & -9.32 & -9.61 & K3245 & -18.30 & -17.24 \\
\hline x35 & -9.63 & -8.89 & 03245 (E-05) & 6.55 & 6.46 \\
\hline$x 44$ & 3.18 & 3.11 & K1435 & 31.74 & 30.79 \\
\hline$x 45$ & -2.42 & -2.35 & 01435 (E-05) & -9.05 & -20.73 \\
\hline$x 55$ & -2.44 & -2.42 & K1534 & 35.07 & 36.07 \\
\hline x|414 & 0.82 & 0.76 & K4455 & -8.58 & -8.61 \\
\hline$x \mid 415$ & 6.55 & 6.54 & 04455 (E-05) & 3.92 & 16.35 \\
\hline x|5|5 & 3.54 & 3.53 & Kp4455 & 4.88 & 4.70 \\
\hline
\end{tabular}


Table 8

\begin{tabular}{|c|c|c|c|c|c|c|}
\hline & ${ }^{12} \mathrm{C}_{2} \mathrm{H}_{2}{ }^{\mathrm{VR}}$ & ${ }^{12} \mathrm{C}_{2} \mathrm{H}_{2}{ }^{\mathrm{V}}$ & ${ }^{13} \mathrm{CH}^{12} \mathrm{CH}^{\mathrm{VR}}$ & ${ }^{13} \mathrm{CH}^{12} \mathrm{CH}^{\mathrm{V}}$ & ${ }^{13} \mathrm{C}_{2} \mathrm{H}_{2}{ }^{\mathrm{V}}$ & ${ }^{12} \mathrm{C}_{2} \mathrm{D}_{2}{ }^{\mathrm{V}}$ \\
\hline$x_{11}$ & -27.2 & -24.8 & -27.3 & -27.0 & -25.4 & -12.3 \\
\hline$x_{33}$ & -25.4 & -27.6 & -27.0 & -27.6 & -26.7 & -15.4 \\
\hline$x_{13}$ & -104.5 & -107.5 & -101.3 & -105.8 & -104.3 & -47.5 \\
\hline$K_{11 / 33}$ & -113.5 & -105.7 & -111.4 & -111.4 & -103.4 & -47.2 \\
\hline
\end{tabular}

Constants are from: ${ }^{12} \mathrm{C}_{2} \mathrm{H}_{2}^{\mathrm{VR}}$, this work; ${ }^{12} \mathrm{C}_{2} \mathrm{H}_{2}^{\mathrm{V}}$, Idrissi et al. ${ }^{43} ;{ }^{13} \mathrm{CH}^{12} \mathrm{CH}^{\mathrm{VR}}$, Fayt et al. ${ }^{9} ;{ }^{13} \mathrm{CH}^{12} \mathrm{CH}^{\mathrm{V}}$, Robert et al. ${ }^{46} ;{ }^{13} \mathrm{C}_{2} \mathrm{H}_{2}$, Di Lonardo et al. ${ }^{44} ;{ }^{12} \mathrm{C}_{2} \mathrm{D}_{2}{ }^{\mathrm{v}}$, Herman et al. ${ }^{42}$. 


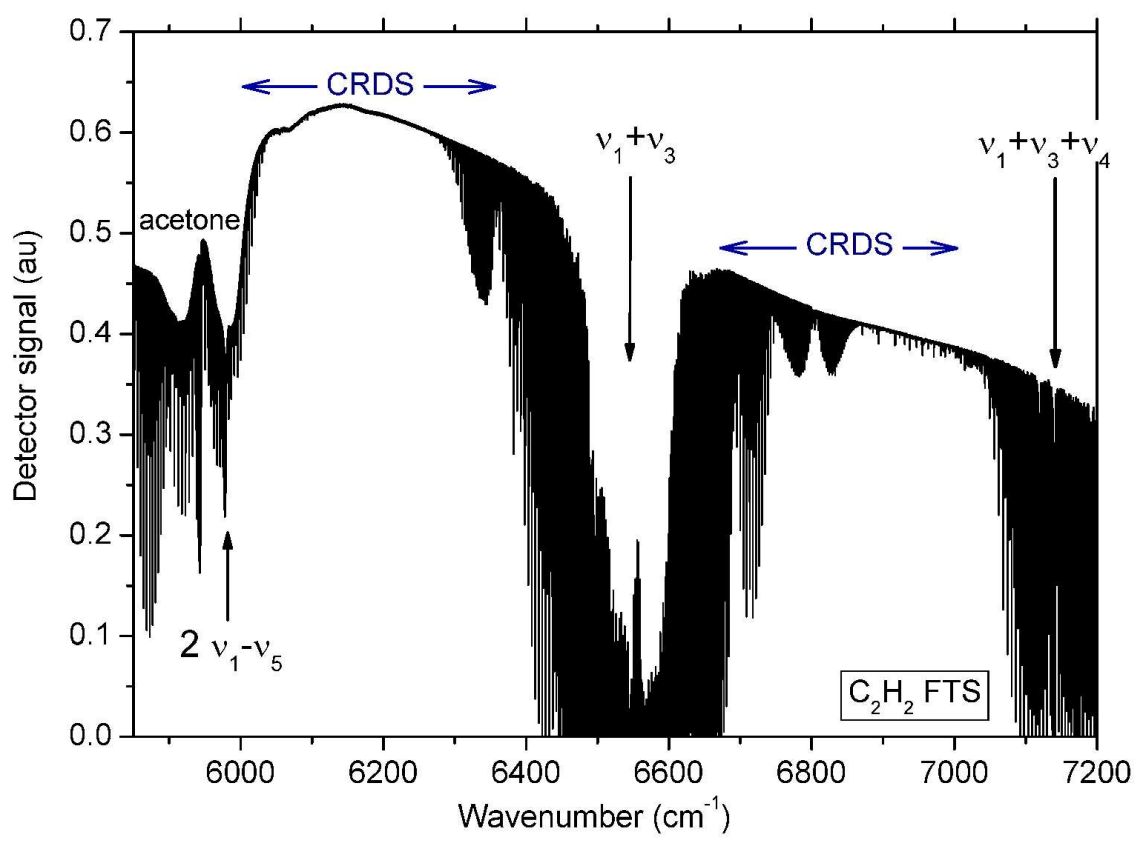

Fig. 1

$297 \times 209 \mathrm{~mm}(300 \times 300$ DPI $)$

34

35

36

37

38

39

40

41

42

43

44

45

46

47

48

49

50

51

52

53

54

55

56

57

58

59

60

URL: http://mc.manuscriptcentral.com/tandf/tmph 


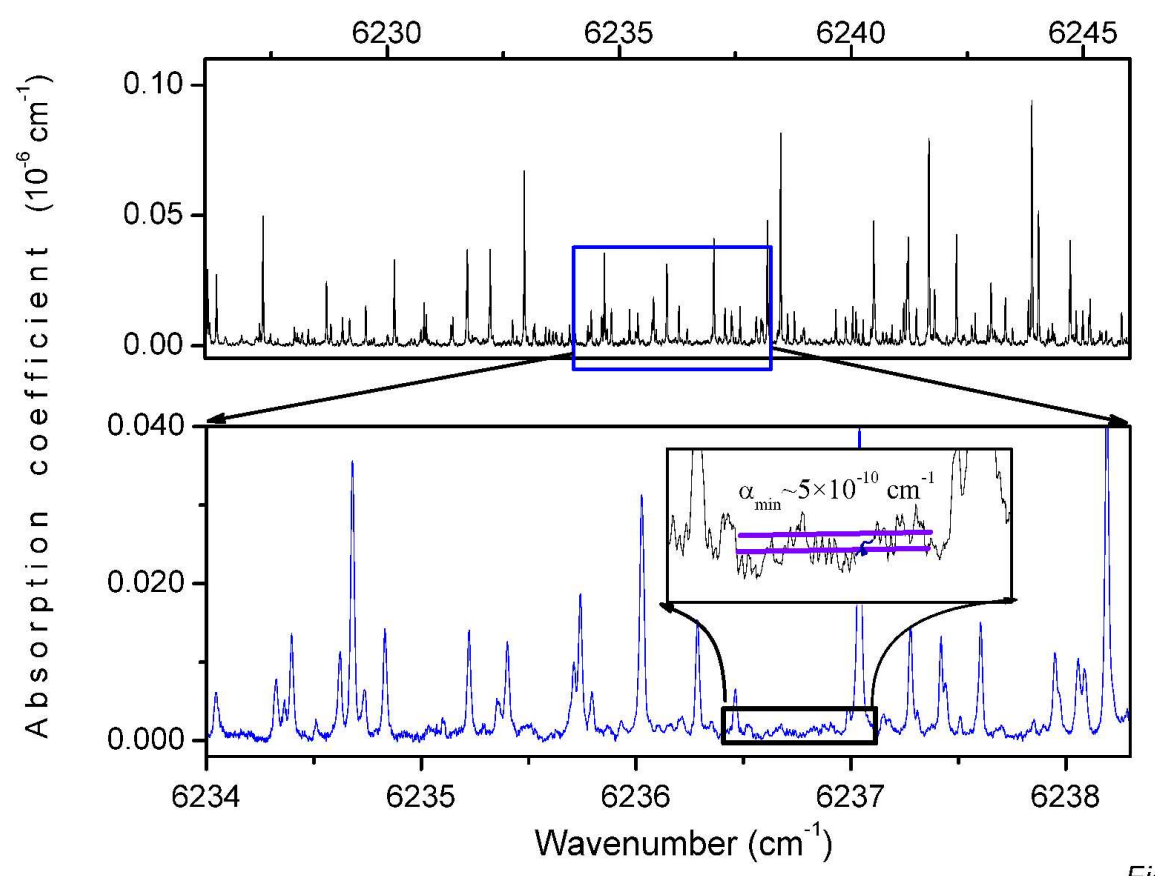

Fig. 2

$297 \times 209 \mathrm{~mm}(300 \times 300$ DPI $)$ 


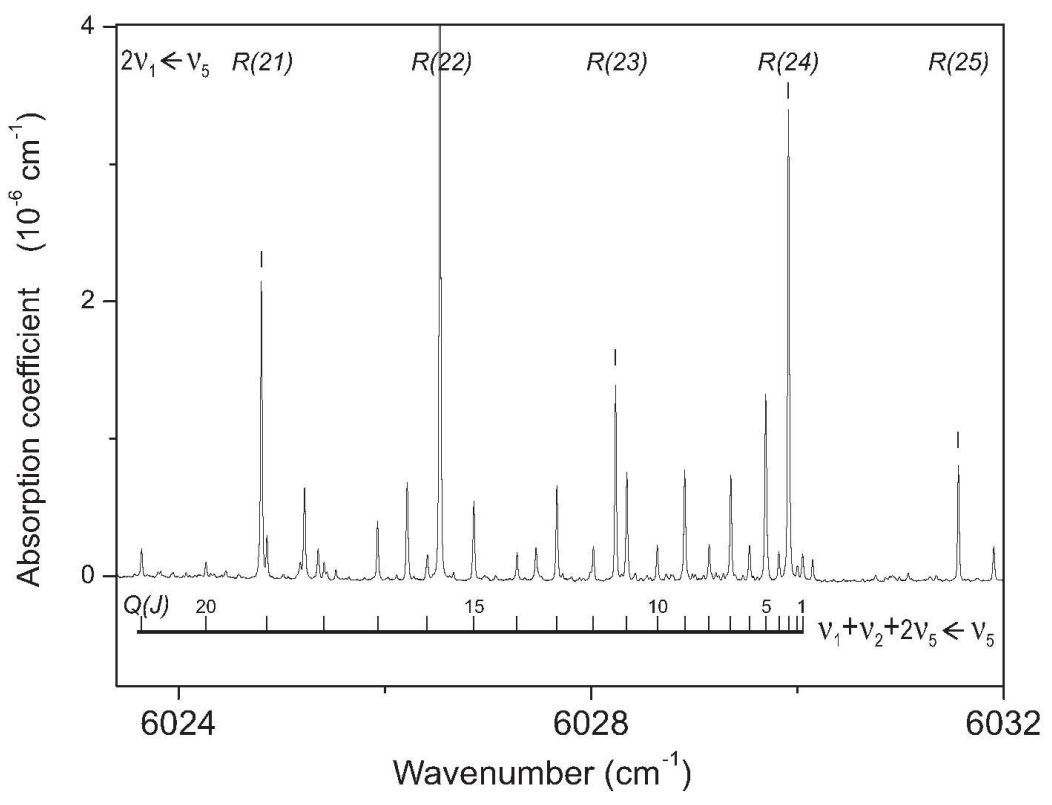

$297 \times 209 m m(300 \times 300$ DPI $)$

1

2

3

4

5

6

9

10

11

12

13

16

17

18

19

20

21

23

24

25

26

27

28

33

34

35

36

37

38

39

40

41

42

43

44

45

46

47

48

49

50

51

52

53

54

55

56

57

58

59

60

URL: http://mc.manuscriptcentral.com/tandf/tmph 


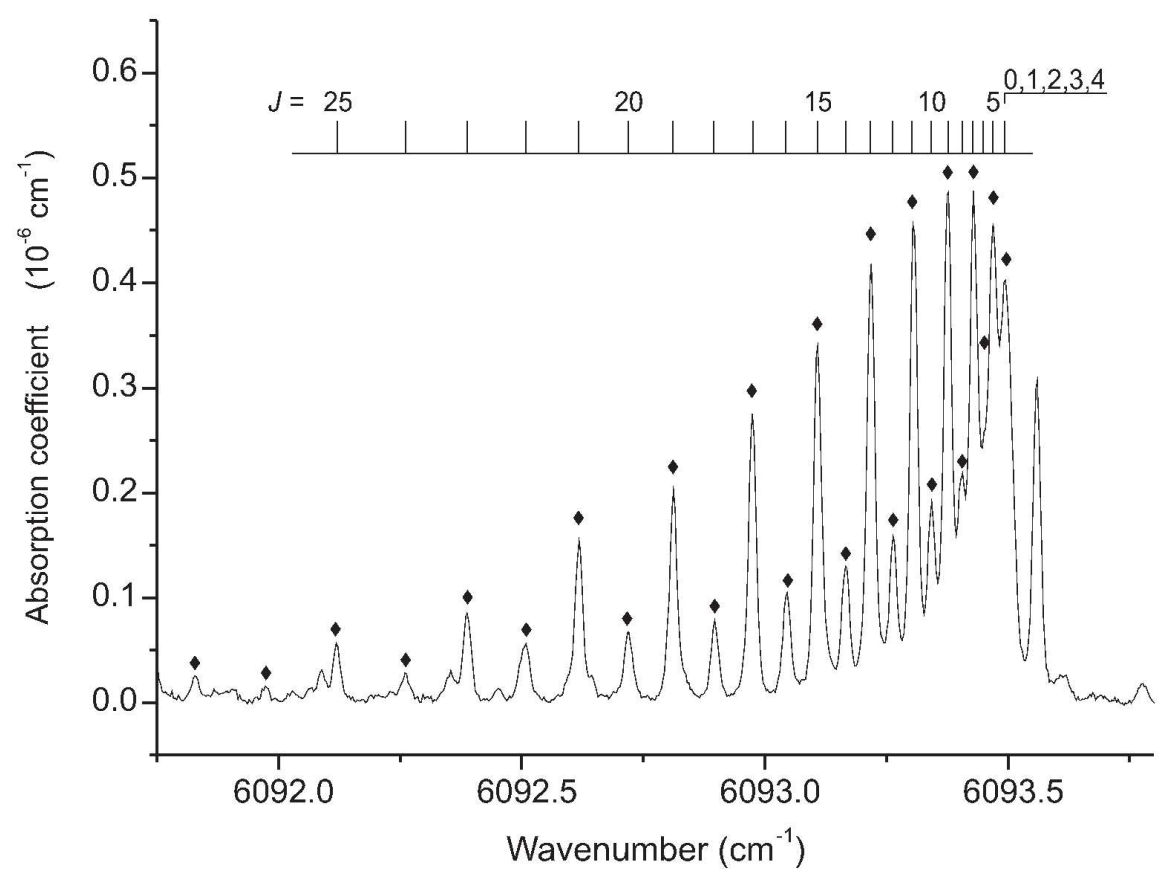

$297 \times 209 \mathrm{~mm}(300 \times 300$ DPI $)$ 


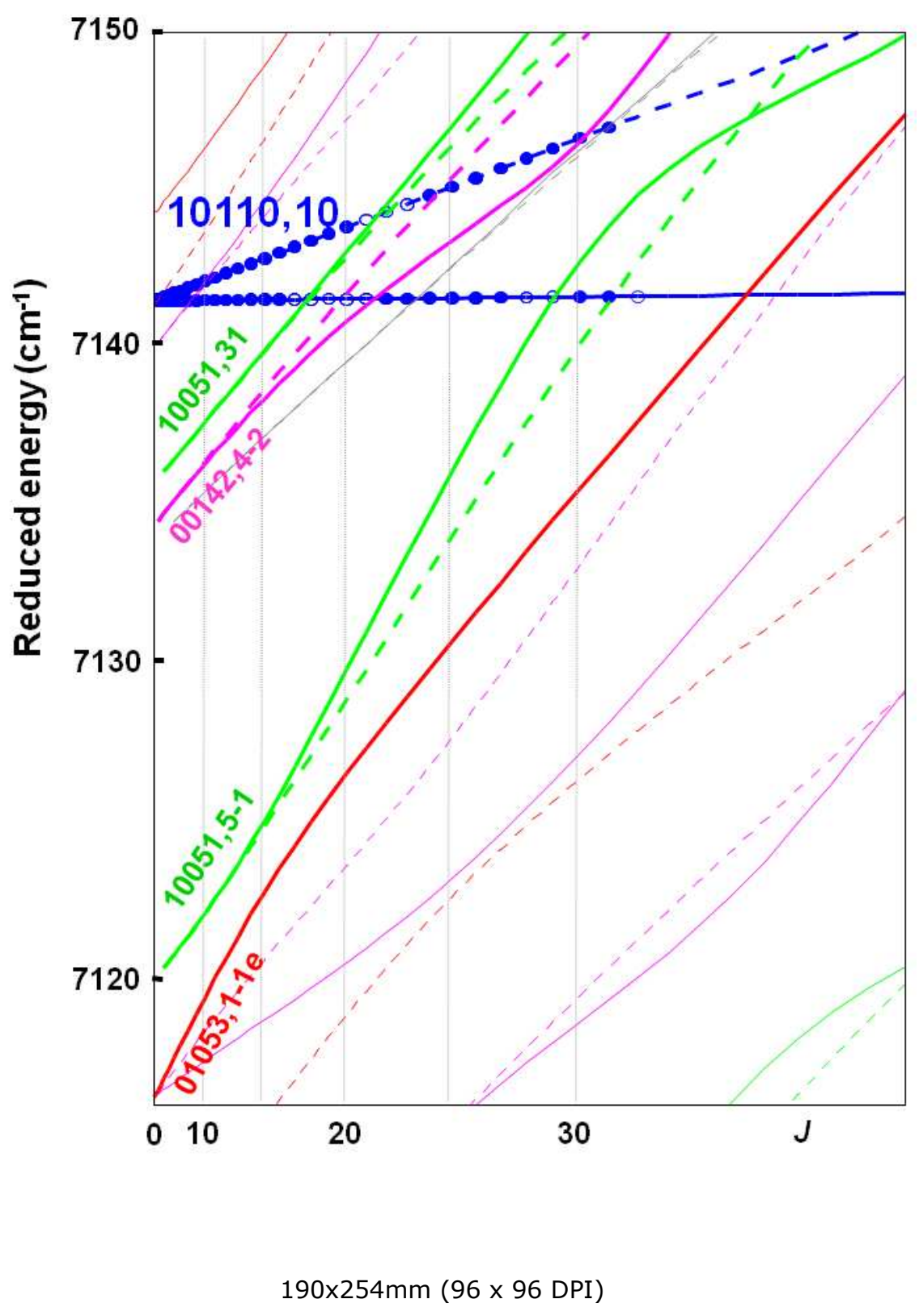

URL: http://mc.manuscriptcentral.com/tandf/tmph 
Molecular Physics: Supplementary Materials (29 pp) for the paper

Acetylene, ${ }^{12} \mathrm{C}_{2} \mathrm{H}_{2}$ : New CRDS data and global vibration-rotation analysis up to $8600 \mathrm{~cm}^{-1}$

by

S. Robert, M. Herman, A. Fayt, A. Campargue, S. Kassi, A. Liu, L. Wang, G. Di Lonardo, and L. Fusina

Table of the bands newly reported from CRDS spectra (rob08), or including updated information with respect to the literature (kep96).

Assignment, experimental line wavenumbers $\left(\mathrm{cm}^{-1}\right)$, uncertainties (in unit of $10^{-3} \mathrm{~cm}^{-1}$ ), observed-calculated values from the global fit procedure (in unit of $10^{-3} \mathrm{~cm}^{-1}$ ) are provided.

(kep96): K. A. Keppler, G. C. Mellau, S. Klee, B. P. Winnewisser, M.

Winnewisser, J. Plíva, and K. N. Rao, J. Mol. Spectrosc. 175, 411 (1996).

(rob08): This work 


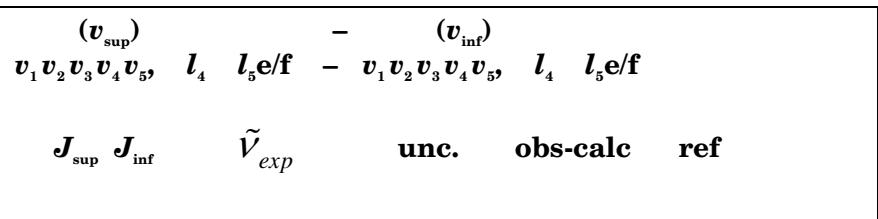

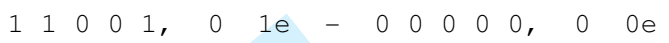

\begin{tabular}{rrllrl}
3 & 2 & 6058.97591 & 0.90 & -1.51 & rob08 \\
4 & 3 & 6061.22752 & 0.0 & 2.38 & rob08 \\
5 & 4 & 6063.44578 & 0.90 & -0.59 & rob08 \\
6 & 5 & 6065.64183 & 0.90 & 0.77 & rob08 \\
7 & 6 & 6067.80822 & 0.90 & -0.97 & rob08 \\
8 & 7 & 6069.94999 & 0.90 & -0.71 & rob08 \\
9 & 8 & 6072.06539 & 0.90 & -0.17 & rob08 \\
10 & 9 & 6074.15383 & 0.90 & 0.10 & rob08 \\
11 & 10 & 6076.21437 & 0.90 & -0.79 & rob08 \\
12 & 11 & 6078.25094 & 0.90 & 1.12 & rob08 \\
13 & 12 & 6080.25679 & 0.90 & -0.88 & rob08 \\
14 & 13 & 6082.23784 & 0.90 & -0.82 & rob08 \\
15 & 14 & 6084.19240 & 0.90 & -0.35 & rob08 \\
16 & 15 & 6086.11963 & 0.90 & -0.27 & rob08 \\
17 & 16 & 6088.01995 & 0.90 & -0.12 & rob08 \\
18 & 17 & 6089.89309 & 0.90 & -0.13 & rob08 \\
19 & 18 & 6091.73890 & 0.90 & -0.40 & rob08 \\
20 & 19 & 6093.55914 & 0.90 & 0.86 & rob08 \\
21 & 20 & 6095.35221 & 0.0 & 2.09 & rob08 \\
22 & 21 & 6097.11498 & 0.90 & 0.20 & rob08 \\
23 & 22 & 6098.85154 & 0.90 & -0.66 & rob08 \\
24 & 23 & 6100.56348 & 0.90 & 1.11 & rob08 \\
25 & 24 & 6102.24472 & 0.90 & -0.50 & rob08 \\
26 & 25 & 6103.90071 & 0.90 & -0.02 & rob08 \\
27 & 26 & 6105.52791 & 0.90 & -0.94 & rob08 \\
28 & 27 & 6107.12924 & 0.90 & -0.30 & rob08 \\
29 & 28 & 6108.70111 & 0.90 & -1.65 & rob08 \\
30 & 29 & 6110.24687 & 0.90 & -1.61 & rob08 \\
& & & & & \\
\hline
\end{tabular}

$\begin{array}{llllllllllllllll}1 & 1 & 0 & 0 & 1, & 0 & 1 \mathrm{f} & - & 0 & 0 & 0 & 0 & 0, & 0 & 0 e\end{array}$

$\begin{array}{rrrrrr}1 & 1 & 6052.05776 & 0.70 & -1.39 & \text { rob08 } \\ 2 & 2 & 6052.02657 & 0.70 & 0.56 & \text { rob08 } \\ 3 & 3 & 6051.97660 & 0.70 & 0.30 & \text { rob08 } \\ 4 & 4 & 6051.90904 & 0.70 & -0.97 & \text { rob08 } \\ 5 & 5 & 6051.82618 & 0.70 & -0.96 & \text { rob08 } \\ 6 & 6 & 6051.72780 & 0.70 & 0.14 & \text { rob08 } \\ 7 & 7 & 6051.61096 & 0.70 & -0.62 & \text { rob08 } \\ 8 & 8 & 6051.47822 & 0.70 & -0.66 & \text { rob08 } \\ 9 & 9 & 6051.32920 & 0.70 & -0.34 & \text { rob08 } \\ 10 & 10 & 6051.16482 & 0.70 & 1.28 & \text { rob08 } \\ 11 & 11 & 6050.98053 & 0.70 & -0.33 & \text { rob08 } \\ 12 & 12 & 6050.78204 & 0.70 & 0.54 & \text { rob08 } \\ 13 & 13 & 6050.56611 & 0.70 & 0.70 & \text { rob08 } \\ 14 & 14 & 6050.33260 & 0.70 & 0.01 & \text { rob08 } \\ 15 & 15 & 6050.08327 & 0.70 & 0.27 & \text { rob08 } \\ 16 & 16 & 6049.81547 & 0.70 & -1.15 & \text { rob08 } \\ 17 & 17 & 6049.53397 & 0.70 & 0.55 & \text { rob08 } \\ 18 & 18 & 6049.23385 & 0.70 & 0.47 & \text { rob08 } \\ 19 & 19 & 6048.91304 & 0.0 & -3.42 & \text { rob08 } \\ 20 & 20 & 6048.58199 & 0.70 & -0.64 & \text { rob08 } \\ 21 & 21 & 6048.23229 & 0.70 & 0.43 & \text { rob08 } \\ 23 & 23 & 6047.47962 & 0.70 & 0.25 & \text { rob08 }\end{array}$

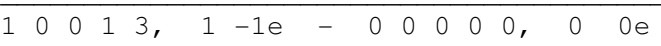




$\begin{array}{llrl}6077.33968 & 2.00 & 1.00 & \text { rob08 } \\ 6082.05301 & 2.00 & 2.77 & \text { rob08 } \\ 6074.99282 & 2.00 & 2.40 & \text { rob08 } \\ 6084.41406 & 2.00 & 0.79 & \text { rob08 } \\ 6072.64910 & 2.00 & 2.06 & \text { rob08 } \\ 6086.79881 & 0.0 & 18.15 & \text { rob08 } \\ 6070.30978 & 2.00 & 1.57 & \text { rob08 } \\ 6089.15383 & 2.00 & 2.00 & \text { rob08 } \\ 6067.97486 & 2.00 & 1.43 & \text { rob08 } \\ 6091.52758 & 2.00 & 1.57 & \text { rob08 } \\ 6065.64261 & 2.00 & 0.60 & \text { rob08 } \\ 6093.90448 & 2.00 & 2.23 & \text { rob08 } \\ 6063.31340 & 2.00 & 0.31 & \text { rob08 } \\ 6096.28131 & 2.00 & 1.87 & \text { rob08 } \\ 6060.98727 & 2.00 & 1.65 & \text { rob08 } \\ 6098.65897 & 2.00 & 2.69 & \text { rob08 } \\ 6058.65937 & 2.00 & 0.99 & \text { rob08 } \\ 6101.03206 & 2.00 & 0.75 & \text { rob08 } \\ 6056.33095 & 2.00 & 0.94 & \text { rob08 } \\ 6103.40256 & 2.00 & -0.40 & \text { rob08 } \\ 6053.99971 & 2.00 & 0.73 & \text { rob08 } \\ 6105.76986 & 2.00 & 0.37 & \text { rob08 } \\ 6051.66509 & 2.00 & 1.43 & \text { rob08 } \\ 6108.12965 & 2.00 & 0.57 & \text { rob08 } \\ 6049.32029 & 2.00 & -2.01 & \text { rob08 } \\ 6110.47846 & 2.00 & -1.39 & \text { rob08 } \\ 6046.95895 & 0.0 & -14.15 & \text { rob08 } \\ 6112.81825 & 2.00 & -1.64 & \text { rob08 } \\ 6044.61115 & 2.00 & -3.07 & \text { rob08 } \\ 6115.14664 & 2.00 & -0.65 & \text { rob08 } \\ 6042.24021 & 2.00 & -3.62 & \text { rob08 } \\ 6117.45928 & 2.00 & -0.93 & \text { rob08 } \\ 6039.85678 & 2.00 & -3.38 & \text { rob08 } \\ 6119.75534 & 2.00 & -1.57 & \text { rob08 } \\ 6037.45674 & 0.0 & -4.82 & \text { rob08 } \\ 6122.03345 & 2.00 & -2.31 & \text { rob08 } \\ 6035.04278 & 2.00 & -3.70 & \text { rob08 } \\ 6032.60795 & 0.0 & -5.62 & \text { rob08 }\end{array}$

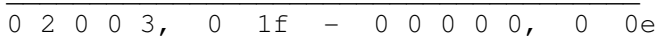

$\begin{array}{rrrrrr}3 & 3 & 6093.49350 & 1.60 & -1.64 & \text { rob08 } \\ 5 & 5 & 6093.46954 & 1.60 & 0.93 & \text { rob08 } \\ 6 & 6 & 6093.45062 & 1.60 & 0.07 & \text { rob08 } \\ 7 & 7 & 6093.42847 & 1.60 & -0.64 & \text { rob08 } \\ 8 & 8 & 6093.40511 & 1.60 & 1.02 & \text { rob08 } \\ 9 & 9 & 6093.37657 & 1.60 & 1.27 & \text { rob08 } \\ 10 & 10 & 6093.34279 & 1.60 & 0.31 & \text { rob08 } \\ 11 & 11 & 6093.30576 & 1.60 & 0.39 & \text { rob08 } \\ 12 & 12 & 6093.26353 & 1.60 & -0.12 & \text { rob08 } \\ 13 & 13 & 6093.21849 & 1.60 & 1.53 & \text { rob08 } \\ 14 & 14 & 6093.16577 & 1.60 & 0.85 & \text { rob08 } \\ 15 & 15 & 6093.10828 & 1.60 & 1.18 & \text { rob08 } \\ 16 & 16 & 6093.04575 & 1.60 & 2.74 & \text { rob08 } \\ 17 & 17 & 6092.97447 & 1.60 & 2.36 & \text { rob08 } \\ 18 & 18 & 6092.89664 & 1.60 & 2.82 & \text { rob08 } \\ 19 & 19 & 6092.81223 & 1.60 & 4.72 & \text { rob08 } \\ 20 & 20 & 6092.71895 & 0.0 & 6.49 & \text { rob08 } \\ 21 & 21 & 6092.61777 & 0.0 & 9.86 & \text { rob08 } \\ 22 & 22 & 6092.50668 & 0.0 & 13.63 & \text { rob08 } \\ 23 & 23 & 6092.38570 & 0.0 & 18.74 & \text { rob08 } \\ 24 & 24 & 6092.25582 & 0.0 & 27.12 & \text { rob08 } \\ 25 & 25 & 6092.11723 & 0.0 & 39.98 & \text { rob08 } \\ 26 & 26 & 6091.97126 & 0.0 & 59.75 & \text { rob08 } \\ 27 & 27 & 6091.82845 & 0.0 & 98.11 & \text { rob08 } \\ 28 & 28 & 6091.66122 & 0.0 & 128.67 & \text { rob08 } \\ 29 & 29 & 6091.50089 & 0.0 & 183.98 & \text { rob08 }\end{array}$

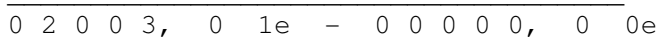

$\begin{array}{llllll}1 & 0 & 6095.83671 & 0.0 & -7.45 & \text { robo8 }\end{array}$ 


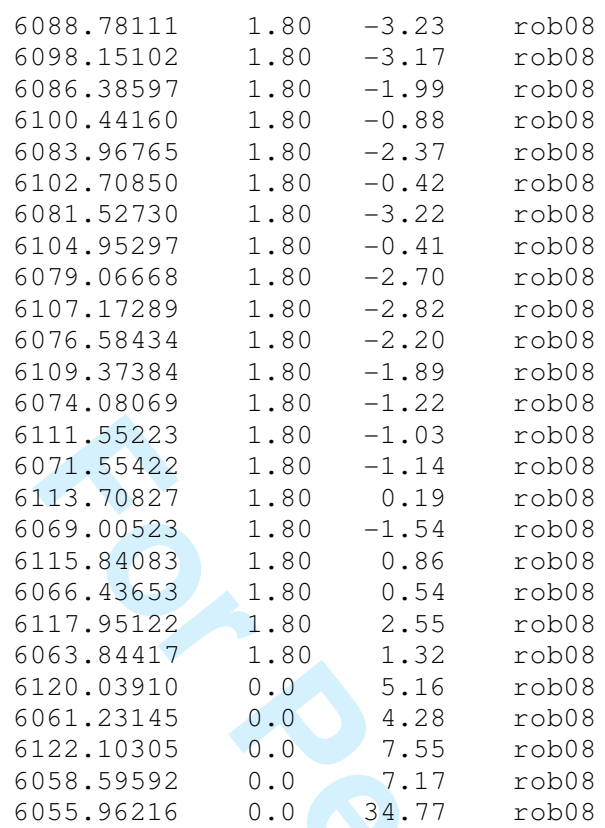




$\begin{array}{rrrlrl}22 & 21 & 6669.89074 & 0.40 & 0.22 & \text { kep96 } \\ 22 & 23 & 6564.14092 & 0.40 & 0.20 & \text { kep96 } \\ 23 & 22 & 6671.73367 & 0.40 & 0.16 & \text { kep96 } \\ 23 & 24 & 6561.29791 & 0.40 & 0.13 & \text { kep96 } \\ 24 & 23 & 6673.54605 & 0.40 & 0.05 & \text { kep96 } \\ 24 & 25 & 6558.42659 & 0.40 & 0.45 & \text { kep96 } \\ 25 & 24 & 6675.32742 & 0.40 & 0.15 & \text { kep96 } \\ 25 & 26 & 6555.52559 & 0.40 & 0.37 & \text { kep96 } \\ 26 & 25 & 6677.07676 & 0.40 & 0.08 & \text { kep96 } \\ 26 & 27 & 6552.59372 & 0.40 & -0.70 & \text { kep96 } \\ 27 & 26 & 6678.79368 & 0.40 & 0.05 & \text { kep96 } \\ 28 & 27 & 6680.47797 & 0.40 & 0.40 & \text { kep96 } \\ 28 & 29 & 6546.64155 & 0.40 & 0.40 & \text { kep96 } \\ 29 & 28 & 6682.12777 & 0.40 & -0.27 & \text { kep96 } \\ 30 & 29 & 6683.74429 & 0.40 & -0.31 & \text { kep96 } \\ 31 & 30 & 6685.32728 & 0.40 & 0.36 & \text { kep96 } \\ 31 & 32 & 6537.47461 & 0.0 & -1.47 & \text { kep96 } \\ 32 & 31 & 6686.87453 & 0.40 & -0.16 & \text { kep96 } \\ 33 & 32 & 6688.38772 & 0.40 & 0.04 & \text { kep96 } \\ 34 & 33 & 6689.86548 & 0.40 & -0.24 & \text { kep96 } \\ 35 & 34 & 6691.30841 & 0.40 & -0.26 & \text { kep96 } \\ 36 & 35 & 6692.71575 & 0.40 & -0.70 & \text { kep96 } \\ 37 & 36 & 6694.08917 & 0.40 & 0.15 & \text { kep96 } \\ 38 & 37 & 6695.42938 & 2.00 & 3.01 & \text { rob08 } \\ 39 & 38 & 6696.72987 & 2.00 & 1.35 & \text { rob08 } \\ 40 & 39 & 6697.99818 & 2.00 & 2.66 & \text { rob08 } \\ 41 & 40 & 6699.22990 & 2.00 & 2.48 & \text { rob08 } \\ 42 & 41 & 6700.42696 & 2.00 & 2.65 & \text { rob08 } \\ 43 & 42 & 6701.58742 & 2.00 & 1.14 & \text { rob08 } \\ 44 & 43 & 6702.71495 & 2.00 & 1.53 & \text { rob08 }\end{array}$

$11011, \quad 1-1 e-0000010,110 e$

$0 \quad 1 \quad 6016.10304$

6006.72362

6016.10076

6004.33904

6018.40521

6020.69743

6022.97640

5997.11636

6025.23100

5994.67859

6027.47457

5992.22111

6029.70155

5989.74831

6031.91245

5987.26064

6034.10508

5984.75745

6036.28031

5982.23562

6038.43460

6040.57190

6042.68903

5974.56079

6044.78576

5971.96409

6046.85959

5969.34694

6048.91276

5966.70836

6050.94098

6052.94661

5961.36734

6054.92695

5958.66259

0.07010 .98

$0.0-3.10$

$1.00 \quad 1.10$

$0.0-7.62$

$0.40-0.53$

$0.40 \quad 0.81$

$0.0 \quad 4.32$

$0.40-0.85$

$0.40-0.79$

$0.0 \quad 1.52$

$0.40-0.84$

$0.40-0.22$

$0.40 \quad-0.97$

$0.0-1.38$

$0.40 \quad-0.21$

$0.40-1.09$

$0.40-0.24$

$0.40 \quad 0.41$

$0.40 \quad 0.37$

$0.40 \quad 0.48$

$0.0-1.30$

$0.40-0.66$

$0.40-0.20$

$0.40 \quad 0.16$

$0.40 \quad 0.57$

$0.40-0.06$

$0.40-0.07$

$0.40-0.48$

$0.40 \quad 0.88$

$0.0-1.37$

$0.40-0.07$

$0.40 \quad 0.28$

$0.0-1.23$

$0.40 \quad 0.01$

$0.40-1.04$

$0.0-3.55$

$0.40 \quad 0.09$

$0.0-1.50$

kep96

kep96

rob0 8

rob0 8

kep96

kep96

kep96

kep96

kep96

kep96

kep96

kep96

kep96

kep96

kep96

kep 96

kep96

kep 96

kep96

kep96

kep96

kep96

kep96

kep96

kep96

kep96

kep96

kep96

kep96

kep96

kep96

kep 96

kep96

kep96

kep96

kep96

kep96

5955.93489

6058.80939

kep96 


$\begin{array}{rrrlrl}22 & 23 & 5953.18371 & 0.0 & 2.32 & \text { kep96 } \\ 23 & 22 & 6060.71107 & 0.0 & -1.60 & \text { kep96 } \\ 24 & 23 & 6062.58446 & 0.0 & -2.21 & \text { kep96 } \\ 25 & 24 & 6064.43089 & 1.00 & -1.30 & \text { rob08 } \\ 26 & 25 & 6066.24748 & 1.00 & -1.11 & \text { rob08 } \\ 27 & 26 & 6068.03331 & 1.00 & -1.97 & \text { rob08 } \\ 28 & 27 & 6069.79239 & 1.00 & 0.66 & \text { rob08 }\end{array}$

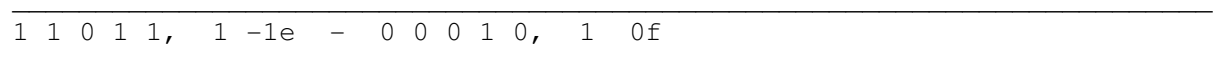

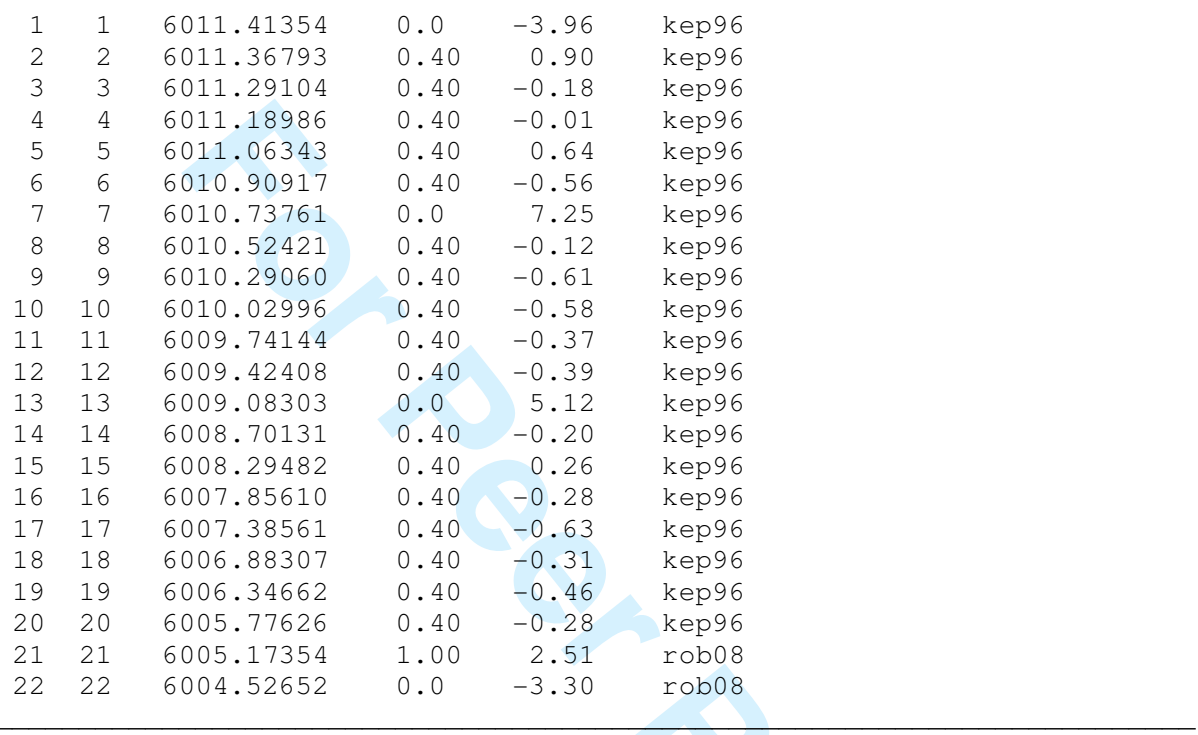

$02013,1-1 e-000000,000$

126649.53387

6658.92994

6647.16313

6661.25277

6644.78207

6663.56640

6642.38663

6665.86613

6639.98224

6668.15470

6637.56539

6670.43031

6672.69184

$0.0-2.45$

$0.40 \quad 0.36$

$0.40-0.22$

$0.40 \quad 0.08$

$0.0 \quad 1.84$

$0.0 \quad 1.42$

$0.40 \quad 0.05$

$0.40 \quad 0.27$

$0.40 \quad 0.38$

$0.40 \quad 0.15$

$0.40 \quad 0.00$

$0.40 \quad 0.13$

$0.40 \quad 0.14$

$0.40 \quad 0.16$

$0.40-0.03$

6630.23661

6677.16784

6679.37950

0.40

0.40

0.40

0.0

6681.57154

6683.72929

6617.68632

6685.89151

6688.01622

6615.11172

6690.11479

6612.51476

6692.18850

6694.22847

6607.23803

6696.24089

6604.56057

6698.22154

6700.17043

0.40

0.40

0.40

0.40

0.40

0.40

0.0

$0.0-1.32$

$0.40-0.06$

0.01 .38

$0.40-0.35$

$0.40 \quad 0.05$

$0.40-0.14$

$2.00 \quad 1.51$

kep 96

kep96

kep96

kep96

kep96

kep96

kep96

kep96

kep96

kep96

kep96

kep96

kep96

kep96

kep96

kep96

kep96

kep96

kep96

rob08

kep96

kep96

kep96

kep96

kep96

kep96

kep96

kep96

kep96

kep96

kep96

kep96

kep96

6702.08537

2.00

1.47

kep96

rob0 8

6705.81438 


$\begin{array}{rrrrrr}25 & 24 & 6707.62513 & 2.00 & 0.97 & \text { rob08 } \\ 26 & 25 & 6709.40088 & 2.00 & 0.92 & \text { rob08 } \\ 27 & 26 & 6711.14099 & 2.00 & 0.85 & \text { rob08 } \\ 28 & 27 & 6712.84552 & 2.00 & 0.95 & \text { rob08 } \\ 29 & 28 & 6714.51362 & 2.00 & 0.40 & \text { rob08 } \\ 30 & 29 & 6716.14627 & 2.00 & 0.16 & \text { rob08 } \\ 31 & 30 & 6717.74298 & 2.00 & -0.32 & \text { rob08 } \\ 32 & 31 & 6719.30521 & 2.00 & 0.32 & \text { rob08 }\end{array}$

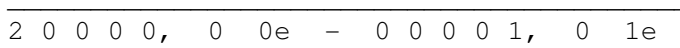

$\begin{array}{llllll}0 & 1 & 5977.51427 & 0.40 & 0.18 & \text { kep96 }\end{array}$

$\begin{array}{llllll}1 & 2 & 5975.13466 & 0.40 & 0.10 & \text { kep96 }\end{array}$

$\begin{array}{llllll}2 & 3 & 5972.72841 & 0.40 & 0.00 & \text { kep96 }\end{array}$

$\begin{array}{llllll}3 & 2 & 5986.76541 & 0.40 & 0.14 & \text { kep96 }\end{array}$

$345970.29571 \quad 0.40 \quad 0.02 \quad k$ kep96

$\begin{array}{rrrrrr}4 & 3 & 5989.01155 & 0.40 & 0.41 & k e p 96 \\ 4 & 5 & 5967.83638 & 0.40 & -0.05 & k e p 96\end{array}$

$\begin{array}{llllll}5 & 4 & 5991.23008 & 0.40 & -0.08 & k e p 96\end{array}$

$\begin{array}{llllll}5 & 6 & 5965.35077 & 0.40 & 0.09 & \text { kep96 } \\ 6 & 5 & 5993.42232 & 0.40 & 0.02 & \text { kep96 }\end{array}$

$\begin{array}{rrrrrr}6 & 7 & 5962.83851 & 0.40 & 0.03 & k e p 96 \\ 7 & 6 & 5995.58737 & 0.40 & -0.15 & k e p 96\end{array}$

$\begin{array}{llllll}7 & 8 & 5960.29986 & 0.40 & -0.01 & \text { kep96 }\end{array}$

$\begin{array}{llllll}8 & 7 & 5997.72582 & 0.40 & 0.03 & \text { kep96 }\end{array}$

$\begin{array}{llllll}8 & 9 & 5957.73491 & 0.40 & 0.01 & k e p 96\end{array}$

$98 \quad 8 \quad 5999.83703 \quad 0.40 \quad-0.05 \quad k$ kep96

$\begin{array}{llllll}9 & 10 & 5955.14358 & 0.40 & -0.03 & k e p 96\end{array}$

$\begin{array}{llllll}10 & 9 & 6001.92151 & 0.40 & 0.16 & \text { kep96 }\end{array}$

$\begin{array}{llllll}10 & 11 & 5952.52645 & 0.40 & 0.41 & \text { kep96 }\end{array}$

$\begin{array}{llllll}11 & 10 & 6003.97849 & 0.40 & -0.07 & \text { kep96 }\end{array}$

$\begin{array}{llllll}11 & 12 & 5949.88214 & 0.40 & -0.11 & k e p 96\end{array}$

$\begin{array}{llllll}12 & 11 & 6006.00900 & 0.40 & 0.30 & \text { kep96 }\end{array}$

$\begin{array}{llllll}12 & 13 & 5947.21227 & 0.40 & 0.01 & \text { kep96 }\end{array}$

$\begin{array}{llllll}13 & 12 & 6008.01175 & 0.40 & 0.03 & k \text { kep96 }\end{array}$

$\begin{array}{llllll}13 & 14 & 5944.51607 & 0.40 & -0.08 & k e p 96\end{array}$

$\begin{array}{llllll}14 & 13 & 6009.98738 & 0.40 & -0.21 & \text { kep96 }\end{array}$

$\begin{array}{llllll}14 & 15 & 5941.79401 & 0.40 & 0.07 & \text { kep96 }\end{array}$

$\begin{array}{llllll}15 & 14 & 6011.93618 & 0.40 & -0.11 & \text { kep96 }\end{array}$

$\begin{array}{llllll}16 & 15 & 6013.85711 & 0.40 & -0.68 & \text { kep96 }\end{array}$

$\begin{array}{llllll}16 & 17 & 5936.27139 & 0.40 & -0.07 & \text { kep96 }\end{array}$

$\begin{array}{llllll}17 & 16 & 6015.75196 & 0.40 & -0.09 & \text { kep96 }\end{array}$

$\begin{array}{llllll}17 & 18 & 5933.47126 & 0.40 & -0.03 & k e p 96\end{array}$

$\begin{array}{llllll}18 & 17 & 6017.61938 & 0.40 & 0.33 & k e p 96\end{array}$

$\begin{array}{llllll}18 & 19 & 5930.64532 & 0.40 & 0.10 & k e p 96\end{array}$

$\begin{array}{llllll}19 & 18 & 6019.45878 & 0.40 & 0.01 & k e p 96\end{array}$

$\begin{array}{llllll}19 & 20 & 5927.79332 & 0.40 & 0.01 & \text { kep96 }\end{array}$

$\begin{array}{llllll}20 & 19 & 6021.27104 & 1.00 & -0.13 & \text { rob08 }\end{array}$

$\begin{array}{llllll}20 & 21 & 5924.91493 & 0.40 & -0.69 & \text { kep96 }\end{array}$

$\begin{array}{llllll}21 & 20 & 6023.05605 & 0.40 & -0.19 & \text { kep96 }\end{array}$

$\begin{array}{llllll}21 & 22 & 5922.01229 & 0.40 & 0.10 & k e p 96\end{array}$

$\begin{array}{llllll}22 & 21 & 6024.81396 & 1.00 & 0.02 & \text { rob08 }\end{array}$

$\begin{array}{llllll}22 & 23 & 5919.08384 & 0.40 & 0.76 \quad k e p 96\end{array}$

$\begin{array}{llllll}23 & 22 & 6026.54427 & 0.40 & 0.00 & \text { kep96 }\end{array}$

$\begin{array}{llllll}23 & 24 & 5916.12824 & 0.40 & -0.12 & \text { kep96 }\end{array}$

$\begin{array}{llllll}24 & 23 & 6028.24675 & 1.00 & -0.43 & \text { rob08 }\end{array}$

$\begin{array}{llllll}25 & 24 & 6029.92339 & 0.40 & 0.73 & \text { kep96 }\end{array}$

$\begin{array}{llllll}25 & 26 & 5910.14020 & 0.0 & -2.03 & \text { kep96 }\end{array}$

$\begin{array}{llllll}25 & 26 & 5910.14223 & 1.00 & 0.00 & \text { rob08 }\end{array}$

$\begin{array}{llllll}26 & 25 & 6031.56959 & 1.00 & -1.11 & \text { rob08 }\end{array}$

$\begin{array}{llllll}27 & 26 & 6033.18991 & 1.00 & -1.35 & \text { rob08 }\end{array}$

$\begin{array}{llllll}27 & 28 & 5904.05307 & 1.00 & -1.21 & \text { rob08 }\end{array}$

$\begin{array}{llllll}28 & 27 & 6034.78447 & 1.00 & 0.13 & \text { rob08 }\end{array}$

$\begin{array}{llllll}28 & 29 & 5900.97069 & 1.00 & -1.58 & \text { rob08 }\end{array}$

$2928 \quad 6036.35005 \quad 1.00 \quad 0.13 \quad$ rob08

$\begin{array}{llllll}29 & 30 & 5897.86472 & 1.00 & -0.26 & \text { rob08 }\end{array}$

$\begin{array}{llllll}30 & 29 & 6037.88729 & 1.00 & -0.68 & \text { robo8 }\end{array}$

$\begin{array}{llllll}30 & 31 & 5894.73186 & 1.00 & -0.61 & \text { rob08 }\end{array}$

$\begin{array}{llllll}31 & 30 & 6039.39751 & 1.00 & -0.97 & \text { rob08 }\end{array}$

$\begin{array}{llllll}31 & 32 & 5891.57465 & 1.00 & -0.15 & \text { rob08 }\end{array}$

$\begin{array}{llllll}32 & 31 & 6040.88041 & 1.00 & -1.03 & \text { rob08 }\end{array}$ 


$\begin{array}{rrrlrl}32 & 33 & 5888.38947 & 0.0 & -2.56 & \text { rob08 } \\ 33 & 32 & 6042.33565 & 1.00 & -1.18 & \text { rob08 } \\ 33 & 34 & 5885.18526 & 0.0 & 1.02 & \text { rob08 } \\ 34 & 33 & 6043.76360 & 1.00 & -1.04 & \text { rob08 } \\ 35 & 34 & 6045.16427 & 1.00 & -0.59 & \text { rob08 } \\ 36 & 35 & 6046.53586 & 1.00 & -1.61 & \text { rob08 } \\ 37 & 36 & 6047.88156 & 1.00 & -0.91 & \text { rob08 }\end{array}$

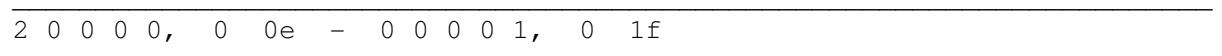

$\begin{array}{llllll}1 & 1 & 5979.83104 & 0.40 & 0.16 & k e p 96 \\ 2 & 2 & 5979.75879 & 0.40 & 0.09 & k e p 96\end{array}$

kep96

$5979.50599 \quad 0.40 \quad-0.08 \quad k \quad k e p 96$

$\begin{array}{llll}5979.32567 & 0.40 & 0.02 & k e p 96\end{array}$

$5979.10920 \quad 0.40 \quad 0.05 \quad$ kep96

$\begin{array}{llll}5978.85662 & 0.40 & 0.02 & \text { kep96 }\end{array}$

$\begin{array}{lllll}5978.56795 & 0.40 & -0.04 & 0.0496\end{array}$

$\begin{array}{llll}5978.24338 & 0.40 & 0.03 & \text { kep96 }\end{array}$

$\begin{array}{llll}5977.88276 & 0.40 & 0.06 & \text { kep96 }\end{array}$

$\begin{array}{llll}5977.48605 & 0.40 & 0.02 & \text { kep96 }\end{array}$

$\begin{array}{llll}5977.05342 & 0.40 & 0.04 & \text { kep96 }\end{array}$

$\begin{array}{lllll}5976.58463 & 0.40 & -0.12 \quad k e p 96\end{array}$

$\begin{array}{lllll}5976.08015 & 0.40 & -0.03 & k e p 96\end{array}$

$\begin{array}{llll}5975.53974 & 0.40 & 0.06 & \text { kep96 }\end{array}$

$\begin{array}{llll}5974.96330 & 0.40 & 0.03 & \text { kep96 }\end{array}$

$\begin{array}{lllll}5974.35099 & 0.40 & -0.01 & \text { kep96 }\end{array}$

$\begin{array}{llll}5973.70265 & 0.40 & -0.22 & \text { kep96 }\end{array}$

$\begin{array}{lllll}5973.01885 & 0.40 & -0.06 \quad k e p 96\end{array}$

$\begin{array}{llll}5972.29919 & 0.40 & 0.01 & \text { kep96 }\end{array}$

$\begin{array}{llll}5971.54378 & 0.40 & 0.11 & k \text { kep96 }\end{array}$

$\begin{array}{llll}5970.75243 & 0.40 & -0.02 \quad k e p 96\end{array}$

$\begin{array}{llll}5969.92548 & 0.40 & -0.05 \quad k e p 96\end{array}$

$\begin{array}{llll}5968.16492 & 0.40 & 0.12 \quad \mathrm{kep} 96\end{array}$

$\begin{array}{llll}5967.23193 & 0.40 & 0.87 & \text { kep96 }\end{array}$

$\begin{array}{llll}5966.26190 & 0.40 & 0.11 & \text { kep96 }\end{array}$

$\begin{array}{llll}5965.26233 & 0.0 & 5.29 & \text { rob08 }\end{array}$

$5964.21460 \quad 0.0 \quad-2.26 \quad$ kep96

$\begin{array}{llll}5963.13898 & 1.00 & -2.31 & \text { rob08 }\end{array}$

$\begin{array}{llll}5962.03075 & 1.00 & 0.37 & \text { robo8 }\end{array}$

$\begin{array}{llll}5960.88468 & 1.00 & 0.48 & \text { rob08 }\end{array}$

$5959.70204 \quad 1.00 \quad-0.74 \quad$ rob08

\begin{tabular}{|c|c|c|c|c|c|}
\hline 1 & 00 & $0 e$ & 00 & 1, & \\
\hline 1 & 2 & 6025.36402 & 1.60 & 0.57 & rob0 8 \\
\hline 2 & 1 & 6034.74322 & 1.60 & 0.64 & rob0 8 \\
\hline 2 & 3 & 6022.97521 & 1.60 & -3.19 & rob0 8 \\
\hline 3 & 2 & 6037.04731 & 1.60 & 0.57 & rob0 8 \\
\hline 3 & 4 & 6020.57831 & 1.60 & 1.15 & rob0 8 \\
\hline 4 & 3 & 6039.33423 & 1.60 & -0.05 & rob0 8 \\
\hline 4 & 5 & 6018.16161 & 1.60 & 2.04 & rob08 \\
\hline 5 & 4 & 6041.60482 & 1.60 & -0.08 & rob08 \\
\hline 6 & 5 & 6043.85769 & 1.60 & -0.54 & rob0 8 \\
\hline 6 & 7 & 6013.27332 & 1.60 & -1.09 & rob08 \\
\hline 7 & 6 & 6046.09495 & 1.60 & 1.09 & rob08 \\
\hline 7 & 8 & 6010.80698 & 1.60 & 0.77 & rob0 8 \\
\hline 8 & 7 & 6048.31212 & 1.60 & 0.83 & rob0 8 \\
\hline 8 & 9 & 6008.31921 & 1.60 & -1.18 & rob0 8 \\
\hline 9 & 8 & 6050.51224 & 1.60 & 2.28 & rob08 \\
\hline 9 & 10 & 6005.81499 & 1.60 & -1.50 & robo 8 \\
\hline 10 & 9 & 6052.69018 & 1.60 & 0.91 & rob0 8 \\
\hline 11 & 10 & 6054.84970 & 1.60 & 1.15 & rob08 \\
\hline 12 & 11 & 6056.98862 & 1.60 & 1.54 & rob08 \\
\hline 13 & 12 & 6059.10540 & 1.60 & 1.31 & rob08 \\
\hline 14 & 13 & 6061.19908 & 1.60 & 0.30 & rob08 \\
\hline 15 & 14 & 6063.27034 & 1.60 & 0.03 & rob08 \\
\hline 16 & 15 & 6065.31774 & 1.60 & -0.09 & rob08 \\
\hline 17 & 16 & 6067.34085 & 1.60 & 0.37 & rob0 8 \\
\hline 18 & 17 & 6069.33758 & 1.60 & 0.20 & rob0 8 \\
\hline 19 & 18 & 6071.30780 & 1.60 & 0.09 & rob08 \\
\hline
\end{tabular}




$\begin{array}{rrrlrl}20 & 19 & 6073.25168 & 1.60 & 1.03 & \text { rob08 } \\ 21 & 20 & 6075.16510 & 1.60 & -0.33 & \text { rob08 } \\ 22 & 21 & 6077.03420 & 0.0 & -17.13 & \text { rob08 } \\ 23 & 22 & 6078.90730 & 1.60 & -0.42 & \text { rob08 } \\ 24 & 23 & 6080.73036 & 1.60 & -3.64 & \text { rob08 } \\ 25 & 24 & 6082.52869 & 1.60 & -0.98 & \text { rob08 } \\ 26 & 25 & 6084.29182 & 1.60 & -2.50 & \text { rob08 } \\ 27 & 26 & 6086.02624 & 1.60 & -1.36 & \text { rob08 } \\ 28 & 27 & 6087.72967 & 1.60 & 0.44 & \text { rob08 } \\ 29 & 28 & 6089.39896 & 1.60 & -0.08 & \text { rob08 }\end{array}$

$\begin{array}{lllllllllllllll}1 & 1 & 0 & 0 & 2, & 0 & 0 e & - & 0 & 0 & 0 & 0 & 1, & 0 & 1 \mathrm{f}\end{array}$

$\begin{array}{llllll}1 & 1 & 6030.05968 & 1.00 & -0.09 & \text { robo8 }\end{array}$

$\begin{array}{llllll}2 & 2 & 6030.00839 & 1.00 & -0.29 & \text { robo8 }\end{array}$

$\begin{array}{llllll}3 & 3 & 6029.92262 & 0.0 & -9.27 & \text { rob08 }\end{array}$

$\begin{array}{llllll}4 & 4 & 6029.82927 & 1.00 & 0.06 & \text { rob08 }\end{array}$

$\begin{array}{llllll}5 & 5 & 6029.70219 & 1.00 & 1.81 & \text { rob08 }\end{array}$

$\begin{array}{llllll}6 & 6 & 6029.54522 & 1.00 & 0.14 & \text { rob08 }\end{array}$

$\begin{array}{llllll}7 & 7 & 6029.36335 & 1.00 & 0.42 & \text { rob08 }\end{array}$

$\begin{array}{llllll}8 & 8 & 6029.15408 & 1.00 & 0.59 & \text { rob08 }\end{array}$

$\begin{array}{llllll}9 & 9 & 6028.91663 & 1.00 & 0.39 & \text { robo8 }\end{array}$

$1010 \quad 6028.65123 \quad 1.00 \quad 0.61 \quad$ rob08

$\begin{array}{llllll}11 & 11 & 6028.35620 & 1.00 & 0.18 & \text { rob08 }\end{array}$

$12126028.03180 \quad 1.00 \quad 0.04 \quad$ rob08

$\begin{array}{llllll}13 & 13 & 6027.67728 & 1.00 & 0.15 & \text { rob08 }\end{array}$

$\begin{array}{llllll}14 & 14 & 6027.29204 & 1.00 & 0.67 & \text { rob08 }\end{array}$

$\begin{array}{llllll}15 & 15 & 6026.87340 & 1.00 & -0.30 & \text { rob08 }\end{array}$

$\begin{array}{llllll}16 & 16 & 6026.42368 & 1.00 & 0.36 & \text { rob08 }\end{array}$

$\begin{array}{llllll}17 & 17 & 6025.93981 & 1.00 & 0.39 & \text { rob08 }\end{array}$

$\begin{array}{llllll}18 & 18 & 6025.42158 & 1.00 & 0.38 & \text { robo8 }\end{array}$

$\begin{array}{llllll}19 & 19 & 6024.86791 & 1.00 & 0.05 & \text { rob08 }\end{array}$

$\begin{array}{llllll}20 & 20 & 6024.27767 & 1.00 & -0.98 & \text { rob08 }\end{array}$

$\begin{array}{llllll}21 & 21 & 6023.65301 & 1.00 & 0.15 & \text { rob08 }\end{array}$

$\begin{array}{llllll}22 & 22 & 6022.97540 & 0.0 & -14.44 & \text { rob08 }\end{array}$

$2323 \quad 6022.28833 \quad 1.00 \quad-0.66 \quad$ rob08

$\begin{array}{llllll}24 & 24 & 6021.54899 & 1.00 & -0.80 & \text { rob08 }\end{array}$

$2525 \quad 6020.77048 \quad 1.00 \quad-1.33 \quad$ rob08

$0 \begin{array}{llllllllllllllll} & 0 & 2 & 1 & 0, & 1 & 0 e & - & 0 & 0 & 0 & 0 & 1, & 0 & 1 e\end{array}$

126357.39495

216366.76102

326369.03607

$\begin{array}{lll}4 & 3 & 6371.29029 \\ 5 & 4 & 6373.51629\end{array}$

$\begin{array}{lll}5 & 6 & 6347.63817 \\ 6 & 5 & 6375.71997\end{array}$

$\begin{array}{lll}6 & 7 & 6345.13633\end{array}$

$\begin{array}{ll}7 & 6 \\ 7 & 6377.89738\end{array}$

$\begin{array}{lll}7 & 8 & 6342.60927\end{array}$

876380.05079

6340.05821

6382.17526

6337.48183

$0.0-1.53$

$0.0 \quad 2.78$

$0.40-0.38$

$0.40 \quad 0.68$

$0.0-1.34$

$0.40 \quad 0.02$

$0.40-0.41$

$0.40-0.24$

$0.40-0.35$

$0.40-0.81$

0.01 .25

$0.40-0.44$

$0.40-0.39$

$0.40-0.34$

$0.40-0.44$

$0.40-1.13$

$0.40-0.27$

$0.40-0.12$

$0.40-0.01$

$0.40 \quad 0.09$

$0.40 \quad 0.10$

$0.40 \quad 0.04$

$0.40-0.66$

$0.40 \quad 0.38$

$0.40 \quad 0.65$

$0.40 \quad 0.66$

$0.40 \quad 0.90$

$0.40 \quad 1.13$

$0.0 \quad 1.29$

kep96

kep96

kep96

kep96

kep96

kep96

kep96

kep96

kep96

kep 96

kep96

kep96

kep96

kep96

kep96

kep96

kep96

kep96

kep96

kep96

6390.41961

6392.41372

6324.22112

6321.49249

6396.32431

6318.73822

6398.23843

$0.0 \quad 1.61$

kep96

kep96

kep96

kep96

kep96

kep96

kep96

kep96

kep96

$17 \quad 18 \quad 6315.95799$ 


$\begin{array}{rrrlrl}18 & 17 & 6400.12510 & 0.0 & 1.71 & \text { kep96 } \\ 18 & 19 & 6313.15250 & 0.0 & 2.95 & \text { kep96 } \\ 19 & 20 & 6310.31963 & 0.0 & 3.15 & \text { kep96 } \\ 20 & 19 & 6403.82042 & 0.0 & 7.85 & \text { kep96 } \\ 20 & 21 & 6307.46499 & 0.0 & 7.97 & \text { kep96 } \\ 21 & 20 & 6405.64762 & 0.0 & 32.54 & \text { kep96 } \\ 21 & 22 & 6304.60367 & 0.0 & 32.64 & \text { kep96 } \\ 22 & 21 & 6407.37057 & 0.0 & -18.67 & \text { kep96 } \\ 22 & 23 & 6301.64553 & 0.0 & -12.84 & \text { kep96 } \\ 23 & 22 & 6409.13093 & 0.0 & -3.88 & \text { kep96 } \\ 23 & 24 & 6298.71487 & 0.0 & -4.03 & \text { kep96 } \\ 24 & 23 & 6410.85101 & 0.40 & -0.59 & \text { kep96 } \\ 24 & 25 & 6295.75177 & 0.0 & -0.71 & \text { kep96 } \\ 25 & 24 & 6412.54033 & 0.40 & 0.95 & \text { kep96 } \\ 25 & 26 & 6292.76002 & 0.40 & 1.06 & \text { kep96 } \\ 26 & 27 & 6289.73813 & 2.00 & -0.06 & \text { rob08 } \\ 27 & 26 & 6415.83291 & 0.0 & 5.87 & \text { kep96 } \\ 27 & 28 & 6286.69682 & 0.0 & 6.76 & \text { kep96 } \\ 28 & 29 & 6283.63319 & 0.0 & 18.79 & \text { rob08 } \\ 29 & 30 & 6280.50469 & 0.0 & -6.37 & \text { rob08 } \\ 30 & 31 & 6277.38056 & 2.00 & 0.64 & \text { rob08 } \\ 31 & 32 & 6274.22436 & 2.00 & 3.56 & \text { rob08 } \\ 32 & 33 & 6271.04127 & 0.0 & 7.71 & \text { rob08 } \\ 33 & 34 & 6267.77779 & 0.0 & -40.24 & \text { rob08 } \\ 34 & 35 & 6264.58543 & 0.0 & 11.38 & \text { rob08 } \\ 35 & 36 & 6261.31552 & 0.0 & 14.07 & \text { rob08 }\end{array}$

\begin{tabular}{llllllllllllllll}
\hline & 0 & 2 & 1 & 0, & 1 & $0 f$ & - & 0 & 0 & 0 & 0 & 1, & 0 & $1 f$
\end{tabular}

$2 \quad 1 \quad 6366.79165$

$2 \quad 3 \quad 6354.98266$

6369.09544

6371.37848

6373.64173

6375.88423

6345.17784

6378.10638

6342.67759

6380.30745

6340.15698

6337.61591

6384.64623

6335.05415

6386.78325

6332.47147

6388.89850

6329.86783

6390.99106

6327.24233

6393.06113

6324.59568

6395.10762

6321.92684

6397.13147

6319.23564

6399.12947

6316.52186

6401.10490

6313.78526

$0.40 \quad-0.89 \quad$ kep96

$0.0 \quad 1.27$

$0.40-0.18$

$0.40-0.24$

$0.40 \quad 0.06$

$0.40-0.07$

$0.40-0.57$

$0.40 \quad 0.03$

$0.40-0.29$

$0.40-0.14$

$0.40-0.15$

$0.40-0.06$

$0.40-0.22$

$0.40-0.01$

$0.40-0.21$

$0.40 \quad 0.00$

$0.40 \quad 0.10$

$0.40 \quad 0.19$

$0.40 \quad 0.14$

$0.40-0.07$

$0.40 \quad 0.46$

$0.40 \quad 0.22$

0.40

0.40

0.40

0.40

0.40

0.40

0.40

0.40

0.40

0.40

0.0

6311.02532

6404.98109

6308.24297

6406.87781

6305.43277

6408.75241

6302.60244

6410.59881

6299.74705

6412.42173

0.0

0.0

0.0

0.40

0.40

0.0

0.40

0.40

0.34

0.31

1. 11

0.32

$-0.04$

0.34

0.52

0.44

0.17

0.40

1.39

1.45

$-1.58$

$-1.57$

$-0.85$

$-0.62$

$-2.16$

kep96

kep 96

kep 96

kep96

kep96

kep96

kep96

kep96

kep96

kep96

kep96

kep96

kep96

kep96

kep 96

kep96

kep 96

kep96

kep96

kep96

kep 96

kep96

kep96

kep96

kep96

kep96

kep 96

kep96

kep96

kep96

kep96

kep96

kep96

kep96

kep96

kep 96

kep96

kep96

kep96

kep96

6296.86668

kep96 


$\begin{array}{rrrlrr}25 & 24 & 6414.21270 & 0.0 & -3.72 & \text { kep96 } \\ 25 & 26 & 6293.96146 & 0.40 & -0.39 & \text { kep96 } \\ 26 & 25 & 6415.98538 & 0.0 & 1.92 & \text { kep96 } \\ 26 & 27 & 6291.03064 & 0.0 & -0.78 & \text { kep96 } \\ 27 & 26 & 6417.72360 & 0.40 & 0.67 & \text { kep96 } \\ 27 & 28 & 6288.07536 & 0.40 & -0.17 & \text { kep96 } \\ 28 & 27 & 6419.43606 & 0.0 & 1.57 & \text { kep96 } \\ 28 & 29 & 6285.09406 & 0.40 & 0.14 & \text { kep96 } \\ 29 & 28 & 6421.11999 & 0.0 & 2.20 & \text { kep96 } \\ 29 & 30 & 6282.08593 & 0.0 & -0.41 & \text { kep96 } \\ 30 & 31 & 6279.05515 & 0.0 & 2.62 & \text { kep96 } \\ 31 & 32 & 6275.99562 & 2.00 & 3.36 & \text { rob08 } \\ 32 & 33 & 6272.94931 & 0.0 & 44.07 & \text { rob08 } \\ 33 & 34 & 6269.77634 & 0.0 & -14.87 & \text { rob08 } \\ 34 & 35 & 6266.63883 & 0.0 & -11.06 & \text { rob08 } \\ 35 & 36 & 6263.47113 & 0.0 & -9.87 & \text { rob08 } \\ 36 & 37 & 6260.27984 & 0.0 & -4.37 & \text { rob08 }\end{array}$

$110021,2-1 e-000010,110 e$

$\begin{array}{llllll}2 & 1 & 6621.25078 & 0.0 & 10.35 & \text { robo8 }\end{array}$

$\begin{array}{llllll}3 & 2 & 6623.56704 & 0.0 & 19.17 & \text { robo8 }\end{array}$

$\begin{array}{llllll}3 & 4 & 6607.10343 & 0.0 & 9.49 & \text { rob08 }\end{array}$

$\begin{array}{llllll}4 & 3 & 6625.84788 & 0.0 & 6.18 & \text { rob08 }\end{array}$

$\begin{array}{llllll}4 & 5 & 6604.68728 & 0.40 & 0.16 & \text { kep96 }\end{array}$

$\begin{array}{llllll}5 & 4 & 6628.13092 & 0.0 & 8.31 & \text { kep96 }\end{array}$

$56 \quad 6602.26756 \quad 2.00 \quad-0.18 \quad$ rob08

$\begin{array}{llllll}6 & 5 & 6630.39432 & 0.0 & 2.99 & \text { kep96 }\end{array}$

$\begin{array}{llllll}6 & 7 & 6599.83786 & 0.0 & 1.26 & \text { kep96 }\end{array}$

$\begin{array}{llllll}7 & 6 & 6632.64070 & 0.0 & -7.84 & \text { rob08 }\end{array}$

$\begin{array}{llllll}7 & 8 & 6597.39519 & 0.40 & 0.73 & \text { kep96 }\end{array}$

$\begin{array}{llllll}8 & 7 & 6634.89483 & 0.40 & 0.22 & \text { kep96 }\end{array}$

$\begin{array}{llllll}8 & 9 & 6594.94241 & 0.40 & 0.64 & k 9096\end{array}$

$\begin{array}{llllll}9 & 8 & 6637.12958 & 0.40 & -0.06 \quad k e p 96\end{array}$

$\begin{array}{llllll}10 & 9 & 6639.35296 & 0.40 & -0.44 & k e p 96\end{array}$

$\begin{array}{llllll}10 & 11 & 6589.99654 & 0.0 & -8.58 & \text { rob08 }\end{array}$

$\begin{array}{llllll}11 & 10 & 6641.56502 & 0.40 & -0.46 & \text { kep96 }\end{array}$

$\begin{array}{llllll}11 & 12 & 6587.52020 & 0.40 & -0.49 & \text { kep96 }\end{array}$

$\begin{array}{llllll}12 & 11 & 6643.76483 & 0.40 & -0.56 & \text { kep96 }\end{array}$

$\begin{array}{llllll}12 & 13 & 6585.02279 & 2.00 & -2.19 & \text { rob08 }\end{array}$

$\begin{array}{llllll}13 & 12 & 6645.95480 & 0.0 & 2.21 & \text { kep96 }\end{array}$

$\begin{array}{llllll}13 & 14 & 6582.51701 & 0.40 & -0.54 & \text { kep96 }\end{array}$

$\begin{array}{llllll}14 & 13 & 6648.12626 & 0.40 & -0.27 & \text { kep96 }\end{array}$

$\begin{array}{llllll}14 & 15 & 6579.99803 & 0.40 & 0.10 & k e p 96\end{array}$

$\begin{array}{llllll}15 & 14 & 6650.28701 & 0.40 & 0.34 & \text { kep96 }\end{array}$

$\begin{array}{llllll}15 & 16 & 6577.46639 & 0.40 & 0.75 & \text { kep96 }\end{array}$

$\begin{array}{llllll}16 & 15 & 6652.43353 & 0.40 & 1.07 & \text { kep96 }\end{array}$

$\begin{array}{llllll}16 & 17 & 6574.92091 & 0.40 & 0.68 & \text { kep96 }\end{array}$

$\begin{array}{llllll}17 & 16 & 6654.56507 & 0.0 & 1.71 & \text { kep96 }\end{array}$

$\begin{array}{llllll}18 & 17 & 6656.68151 & 0.0 & 2.68 & k e p 96\end{array}$

$\begin{array}{llllll}19 & 18 & 6658.78305 & 0.0 & 4.67 & k e p 96\end{array}$

$\begin{array}{llllll}20 & 19 & 6660.86868 & 0.0 & 7.14 & \text { kep96 }\end{array}$

$\begin{array}{llllll}21 & 20 & 6662.93761 & 0.0 & 9.62 & \text { kep96 }\end{array}$

$\begin{array}{llllll}22 & 21 & 6664.98005 & 2.00 & 2.59 & \text { robo8 }\end{array}$

$\begin{array}{llllll}23 & 22 & 6667.01836 & 0.0 & 8.42 & \text { kep96 }\end{array}$

$\begin{array}{llllll}24 & 23 & 6669.03489 & 0.0 & 9.33 & \text { kep96 }\end{array}$

$\begin{array}{llllll}25 & 24 & 6671.04860 & 0.0 & 23.83 & \text { rob08 }\end{array}$

$\begin{array}{llllll}26 & 25 & 6673.02694 & 0.0 & 18.73 & \text { rob08 }\end{array}$

$\begin{array}{llllll}27 & 26 & 6674.94180 & 0.0 & -34.96 & \text { rob08 }\end{array}$

$\begin{array}{llllll}28 & 27 & 6676.88280 & 0.0 & -48.65 & \text { robo8 }\end{array}$

\begin{tabular}{rrrrrlrl}
\hline 1 & 1 & 2 & 1, & $2-1 f-$ & 0.01 & 0,1 & $0 f$ \\
& & & & & & \\
2 & 1 & 6621.25078 & 2.00 & -4.14 & rob08 \\
2 & 3 & 6609.45130 & 0.0 & 1.68 & kep96 \\
3 & 2 & 6623.56704 & 2.00 & 1.09 & rob08 \\
3 & 4 & 6607.04032 & 0.0 & 1.50 & kep96 \\
4 & 3 & 6625.84940 & 0.0 & -10.83 & rob08 \\
4 & 5 & 6604.61508 & 0.0 & 3.54 & kep96 \\
5 & 4 & 6628.13207 & 2.00 & -5.45 & rob08 \\
5 & 6 & 6602.16280 & 0.0 & -4.84 & kep96
\end{tabular}




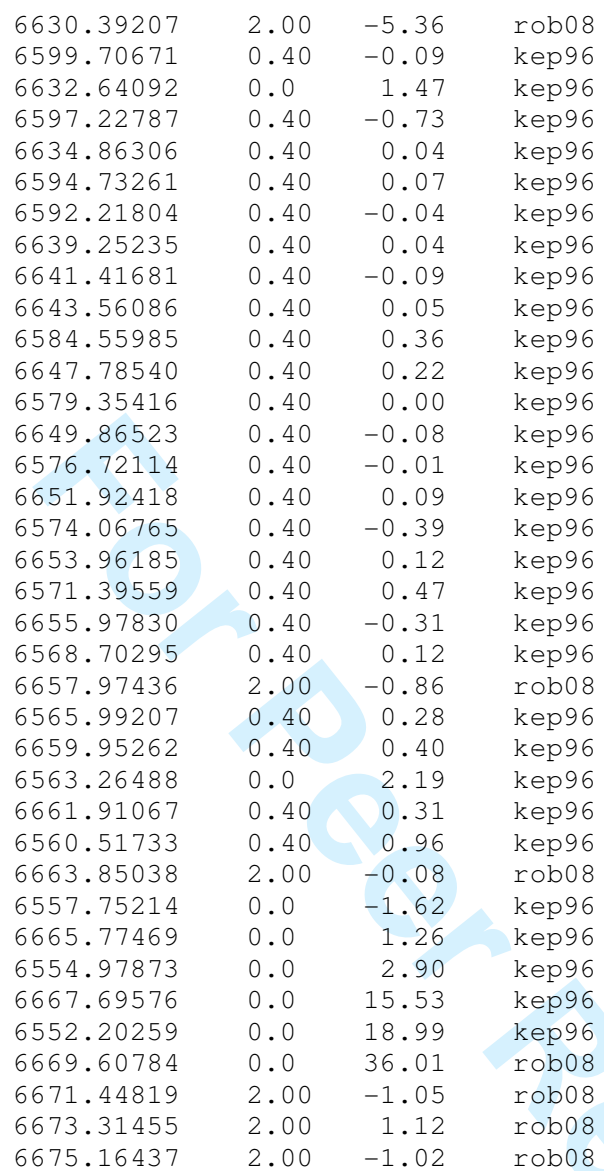
$0.0 \quad 3.80 \quad$ kep96

kep96 kep96 kep 96 kep96 kep96 kep 96 kep96 kep96 kep 96 kep96 kep 96 kep96 kep96 kep96 kep96 kep96 kep96 kep96 kep96 kep96 kep96 kep96 kep96 kep96 kep 96 kep96 kep96 kep96 kep96 


$\begin{array}{rrrlrl}20 & 19 & 6649.59405 & 0.40 & -0.38 & \text { kep96 } \\ 20 & 21 & 6553.33027 & 0.40 & -0.84 & \text { kep96 } \\ 21 & 20 & 6651.53381 & 0.40 & -0.22 & \text { kep96 } \\ 22 & 21 & 6653.45076 & 0.40 & -0.25 & \text { kep96 } \\ 23 & 22 & 6655.34487 & 0.40 & -0.24 & \text { kep96 } \\ 24 & 23 & 6657.21588 & 0.40 & -0.15 & \text { kep96 } \\ 24 & 25 & 6542.22714 & 0.40 & -0.29 & \text { kep96 } \\ 25 & 24 & 6659.06364 & 0.40 & 0.11 & \text { kep96 } \\ 26 & 25 & 6660.88707 & 0.40 & -0.30 & \text { kep96 } \\ 26 & 27 & 6536.54483 & 0.0 & -2.51 & \text { kep96 } \\ 27 & 26 & 6662.68668 & 0.40 & -0.63 & \text { kep96 } \\ 28 & 27 & 6664.46347 & 0.40 & 0.32 & \text { kep96 } \\ 28 & 29 & 6530.77873 & 0.0 & -1.30 & \text { kep96 } \\ 29 & 28 & 6666.21451 & 0.40 & -0.20 & \text { kep96 } \\ 30 & 29 & 6667.94261 & 0.40 & 0.78 & \text { kep96 } \\ 31 & 30 & 6669.64430 & 0.40 & -0.07 & \text { kep96 } \\ 32 & 31 & 6671.34163 & 0.0 & 19.43 & \text { rob08 } \\ 33 & 32 & 6672.97986 & 2.00 & 4.63 & \text { rob08 } \\ 34 & 33 & 6674.60629 & 2.00 & 2.92 & \text { rob08 } \\ 35 & 34 & 6676.20635 & 2.00 & -0.22 & \text { rob08 } \\ 36 & 35 & 6677.78919 & 2.00 & 4.41 & \text { rob08 } \\ 37 & 36 & 6679.34306 & 2.00 & 5.08 & \text { rob08 } \\ 38 & 37 & 6680.87042 & 2.00 & 4.27 & \text { rob08 } \\ 39 & 38 & 6682.37526 & 0.0 & 5.97 & \text { rob08 } \\ 40 & 39 & 6683.85251 & 2.00 & 5.10 & \text { rob08 }\end{array}$

\begin{tabular}{lllllllllllllllll}
\hline 0 & 0 & 2 & 0 & 1, & 0 & $1 \mathrm{f}$ & - & 0 & 0 & 0 & 1 & 0, & 1 & $0 f$
\end{tabular}

$\begin{array}{llllll}3 & 4 & 6596.92189 & 0.40 & 0.18 & \text { kep96 }\end{array}$

$4 \quad 5 \quad 6594.46570$

6617.96094

6591.99039

6620.18050

6589.48976

6586.96543

6584.41832

6626.69694

6628.82147

6579.25259

6630.92096

6576.63605

6632.99702

6573.99527

6635.04749

6571.33063

6637.06901

6568.64018

6639.07518

6565.93120

6641.05185

6563.19551

6643.00377

6560.43867

6644.92820

6646.82999

6648.70500

6650.55436

6667.59530

6669.15117

$0.0-1.81 \quad$ kep96

$0.40 \quad 0.98 \quad k e p 96$

$0.40 \quad 0.32 \quad$ kep96

$0.40 \quad 0.49 \quad$ kep96

$0.40 \quad 0.38 \quad k e p 96$

$0.40 \quad 0.02 \quad$ kep96

$0.40 \quad 0.19 \quad k e p 96$

$0.40 \quad-0.01 \quad k e p 96$

$0.40 \quad 0.32 \quad k e p 96$

$0.40-0.97 \quad$ kep96

$0.40-0.12 \quad$ kep96

$0.40-0.11 \quad$ kep96

$0.40 \quad 0.41 \quad$ kep96

$0.40-0.02 \quad$ kep96

$0.40-0.11 \quad$ kep96

$0.40-0.26 \quad$ kep96

$0.0 \quad-4.90 \quad$ kep96

$0.0-2.71 \quad$ kep96

$0.40-0.21 \quad k e p 96$

$0.40-0.03 \quad k e p 96$

$0.40-0.04 \quad k e p 96$

$0.40-0.33 \quad$ kep 96

$0.40 \quad 0.51 \quad$ kep96

$0.0 \quad 2.03 \quad$ kep96

$0.0 \quad-1.13 \quad k e p 96$

$0.40 \quad 0.03 \quad$ kep96

$0.40 \quad 0.04 \quad k e p 96$

$0.40 \quad 0.15 \quad$ kep96

$2.00 \quad-0.37 \quad$ rob08

$\begin{array}{lll}2.00 & -0.35 \quad \text { rob08 }\end{array}$

$\begin{array}{llll}6670.68019 & 2.00 & 0.32 & \text { rob08 } \\ 6672.18147 & 2.00 & 0.92 & \text { rob08 }\end{array}$

$\begin{array}{llll}6673.65418 & 2.00 & 0.78 & \text { rob08 }\end{array}$

$\begin{array}{llllll}36 & 35 & 6675.09993 & 2.00 & 1.69 & \text { rob08 } \\ 37 & 36 & 6676.51758 & 2.00 & 2.71 & \text { rob08 } \\ 38 & 37 & 6677.90485 & 2.00 & 1.83 & \text { rob08 }\end{array}$

\begin{tabular}{llllllllllllllll}
\hline & 1 & 1 & 1 & 2, & 1 & $0 e$ & - & 0 & 0 & 0 & 1 & 0, & 1 & $0 e$
\end{tabular}

$\begin{array}{llllll}3 & 2 & 6669.01159 & 2.00 & -1.25 & \text { rob08 } \\ 4 & 3 & 6671.28227 & 2.00 & -1.82 & \text { rob08 } \\ 5 & 4 & 6673.51333 & 0.0 & -21.89 & \text { robo } 8\end{array}$

$\begin{array}{llllll}5 & 4 & 6673.51333 & 0.0 & -21.89 & \text { robo8 }\end{array}$ 


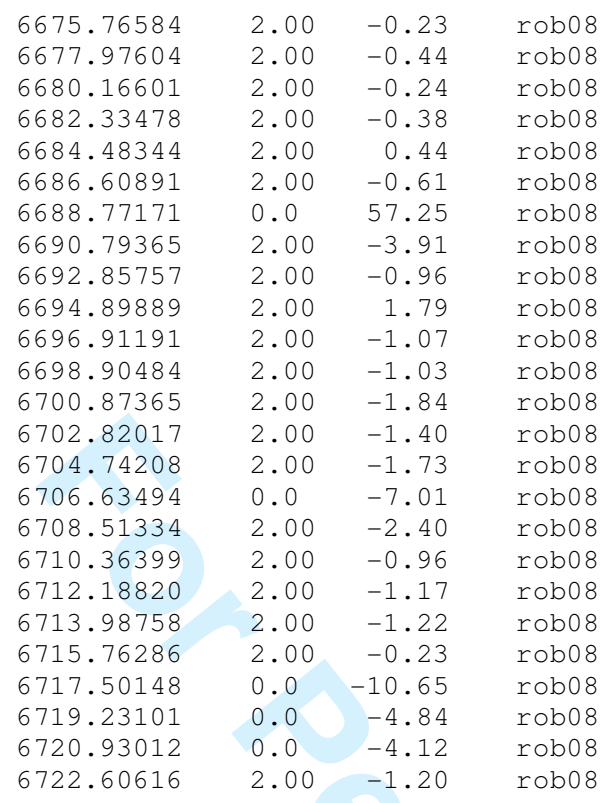

$\begin{array}{rrrrrl}1 & 2 & 6669.68323 & 1.60 & -0.28 & \text { rob08 } \\ 2 & 1 & 6679.08083 & 1.60 & 0.07 & \text { rob08 } \\ 2 & 3 & 6667.27477 & 1.60 & -0.69 & \text { rob08 } \\ 3 & 2 & 6681.37038 & 1.60 & -0.59 & \text { rob08 } \\ 4 & 3 & 6683.63333 & 1.60 & -3.88 & \text { rob08 } \\ 5 & 4 & 6685.86340 & 0.0 & -15.92 & \text { rob08 } \\ 6 & 5 & 6688.09640 & 1.60 & -0.80 & \text { rob08 } \\ 7 & 6 & 6690.28922 & 1.60 & -1.62 & \text { rob08 } \\ 8 & 7 & 6692.45954 & 1.60 & -0.76 & \text { rob08 } \\ 9 & 8 & 6694.60643 & 1.60 & 0.72 & \text { rob08 } \\ 10 & 9 & 6696.72864 & 1.60 & 1.37 & \text { rob08 } \\ 11 & 10 & 6698.82480 & 1.60 & -0.43 & \text { rob08 } \\ 12 & 11 & 6700.90032 & 1.60 & 0.40 & \text { rob08 } \\ 13 & 12 & 6702.95221 & 1.60 & 0.53 & \text { rob08 }\end{array}$




$\begin{array}{rrrrrr}14 & 13 & 6704.98096 & 1.60 & -0.01 & \text { rob08 } \\ 15 & 14 & 6706.98873 & 1.60 & 0.45 & \text { rob08 } \\ 16 & 15 & 6708.97402 & 1.60 & -0.15 & \text { rob08 } \\ 17 & 16 & 6710.93861 & 1.60 & -0.70 & \text { rob08 } \\ 18 & 17 & 6712.88346 & 1.60 & -0.97 & \text { rob08 } \\ 19 & 18 & 6714.80774 & 1.60 & -2.67 & \text { rob08 } \\ 20 & 19 & 6716.71621 & 1.60 & -1.98 & \text { rob08 } \\ 21 & 20 & 6718.60485 & 1.60 & -4.00 & \text { rob08 } \\ 22 & 21 & 6720.47675 & 0.0 & -6.84 & \text { rob08 } \\ 23 & 22 & 6722.33785 & 0.0 & -5.87 & \text { rob08 } \\ 24 & 23 & 6724.18428 & 0.0 & -6.36 & \text { rob08 } \\ 25 & 24 & 6726.01162 & 0.0 & -14.21 & \text { rob08 } \\ 26 & 25 & 6727.83735 & 0.0 & -13.45 & \text { rob08 } \\ 27 & 26 & 6729.64940 & 0.0 & -17.68 & \text { rob08 } \\ 28 & 27 & 6731.44646 & 0.0 & -29.61 & \text { rob08 }\end{array}$

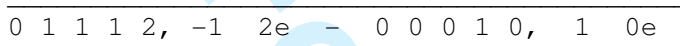

$\begin{array}{llllll}1 & 2 & 6669.72132 & 2.00 & -0.23 & \text { robo8 }\end{array}$

$\begin{array}{llllll}2 & 1 & 6679.11139 & 2.00 & 0.19 & \text { rob08 }\end{array}$

$\begin{array}{llllll}2 & 3 & 6667.35600 & 2.00 & -2.20 & \text { rob08 }\end{array}$

$\begin{array}{llllll}3 & 2 & 6681.44407 & 2.00 & 1.83 & \text { rob08 }\end{array}$

$436683.74000 \quad 0.0 \quad-26.19 \quad$ rob08

$\begin{array}{llllll}5 & 4 & 6686.08706 & 2.00 & 4.51 & \text { robo8 }\end{array}$

$\begin{array}{llllll}6 & 5 & 6688.39338 & 2.00 & 2.69 & \text { rob08 }\end{array}$

$\begin{array}{llllll}7 & 6 & 6690.69376 & 2.00 & 3.95 & \text { rob08 }\end{array}$

$\begin{array}{llllll}8 & 7 & 6692.98328 & 2.00 & 4.23 & \text { rob08 }\end{array}$

$\begin{array}{llllll}9 & 8 & 6695.26351 & 2.00 & 5.88 & \text { rob08 }\end{array}$

$\begin{array}{llllll}10 & 9 & 6697.52659 & 2.00 & 1.60 & \text { rob08 }\end{array}$

$\begin{array}{llllll}11 & 10 & 6699.77714 & 0.0 & -3.72 & \text { rob08 }\end{array}$

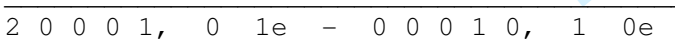

126799.98045

216809.33460

6797.58057

6811.60556

6795.15500

6813.86008

6792.70552

6816.08630

6790.23109

6818.28751

6787.73279

6820.46326

6785.20950

6822.61438

6782.66158

6824.74062

6780.08907

6826.84077

6777.49240

6828.91581

6774.87174

6830.96632

6772.22630

6832.99211

$0.40-0.09$

$0.0 \quad 1.25$

$0.40 \quad 0.22$

$0.0-3.80$

$0.40-0.44$

$0.40-0.34$

$0.40-0.33$

$0.40-0.20$

$0.40-0.53$

$0.40-0.03$

$0.40-0.02$

$0.40-0.28$

$0.40 \quad 0.05$

$0.40-0.05$

$0.40 \quad-0.01$

$0.40 \quad 0.42$

$0.40-0.20$

$0.40-0.05$

$0.40-0.14$

$0.40-0.43$

$0.40 \quad 0.30$

$0.40-0.13$

$0.40 \quad 0.27$

$0.40 \quad 0.72$

$0.40-0.24$

$0.40 \quad 0.33$

$0.40 \quad 0.10$

$0.40-0.13$

$0.40-0.26$

$0.40-0.31$

$0.40-0.33$

$0.40-0.47$

$0.40-0.02$

$\begin{array}{ll}0.40 \quad 0.47 \\ 0.40 & -0.17\end{array}$

$0.40-0.17$

$0.40 \quad 0.27$

0.40

0.27
0.32

kep96

kep96

kep96

kep96

kep96

kep96

kep96

kep96

kep96

kep96

kep96

kep96

kep96

kep96

kep96

kep96

kep96

kep 96

kep96

kep96

kep96

kep96

kep96

kep96

kep96

kep96

kep96

kep96

kep96

kep96

kep96

kep96

kep96

6758.63592

6755.84551

6844.60947

6753.03175

0.40

$-0.64$

kep96

kep96

kep96 


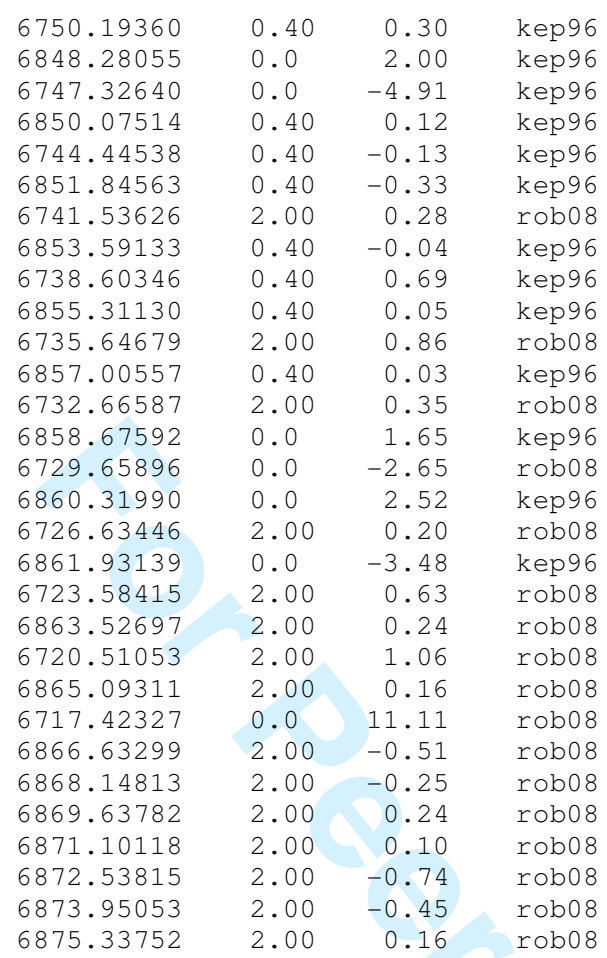




$\begin{array}{rrrlrl}20 & 21 & 6749.82327 & 0.40 & -0.44 & \text { kep96 } \\ 21 & 20 & 6848.33058 & 0.40 & 0.59 & \text { kep96 } \\ 21 & 22 & 6746.94013 & 0.0 & 4.12 & \text { kep96 } \\ 22 & 21 & 6850.12027 & 0.40 & -0.17 & \text { kep96 } \\ 22 & 23 & 6744.02446 & 2.00 & 0.72 & \text { rob08 } \\ 23 & 22 & 6851.88564 & 0.40 & 1.07 & \text { kep96 } \\ 23 & 24 & 6741.08731 & 0.40 & 0.35 & \text { kep96 } \\ 24 & 23 & 6853.62182 & 2.00 & -0.51 & \text { rob08 } \\ 24 & 25 & 6738.12614 & 2.00 & 0.43 & \text { rob08 } \\ 25 & 24 & 6855.33455 & 0.40 & 0.83 & \text { kep96 } \\ 25 & 26 & 6735.14081 & 0.40 & 0.76 & \text { kep96 } \\ 26 & 25 & 6857.00794 & 0.0 & -10.76 & \text { rob08 } \\ 26 & 27 & 6732.12975 & 2.00 & -0.28 & \text { rob08 } \\ 27 & 26 & 6858.67633 & 2.00 & -0.91 & \text { rob08 } \\ 27 & 28 & 6729.09584 & 2.00 & 0.14 & \text { rob08 } \\ 28 & 27 & 6860.30870 & 2.00 & -0.63 & \text { rob08 } \\ 28 & 29 & 6726.03647 & 2.00 & -0.66 & \text { rob08 } \\ 29 & 28 & 6861.91479 & 0.40 & -0.15 & \text { kep96 } \\ 29 & 30 & 6722.94607 & 0.0 & -8.30 & \text { rob08 } \\ 30 & 29 & 6863.49378 & 2.00 & -0.27 & \text { rob08 } \\ 30 & 31 & 6719.84739 & 2.00 & -0.09 & \text { rob08 } \\ 31 & 30 & 6865.04519 & 2.00 & -1.44 & \text { rob08 } \\ 31 & 32 & 6716.71621 & 2.00 & -0.30 & \text { rob08 } \\ 32 & 31 & 6866.56613 & 0.0 & -6.55 & \text { rob08 } \\ 32 & 33 & 6713.56475 & 2.00 & 3.21 & \text { rob08 } \\ 33 & 32 & 6868.07015 & 2.00 & -2.01 & \text { rob08 } \\ 33 & 34 & 6710.38371 & 2.00 & 1.10 & \text { rob08 } \\ 34 & 33 & 6869.54193 & 2.00 & -3.15 & \text { rob08 } \\ 34 & 35 & 6707.17958 & 2.00 & -0.22 & \text { rob08 } \\ 35 & 34 & 6870.98836 & 2.00 & -3.04 & \text { rob08 } \\ 36 & 35 & 6872.40660 & 2.00 & -4.51 & \text { rob08 } \\ 37 & 36 & 6873.80007 & 2.00 & -4.14 & \text { rob08 } \\ 38 & 37 & 6875.16580 & 2.00 & -4.88 & \text { rob08 }\end{array}$

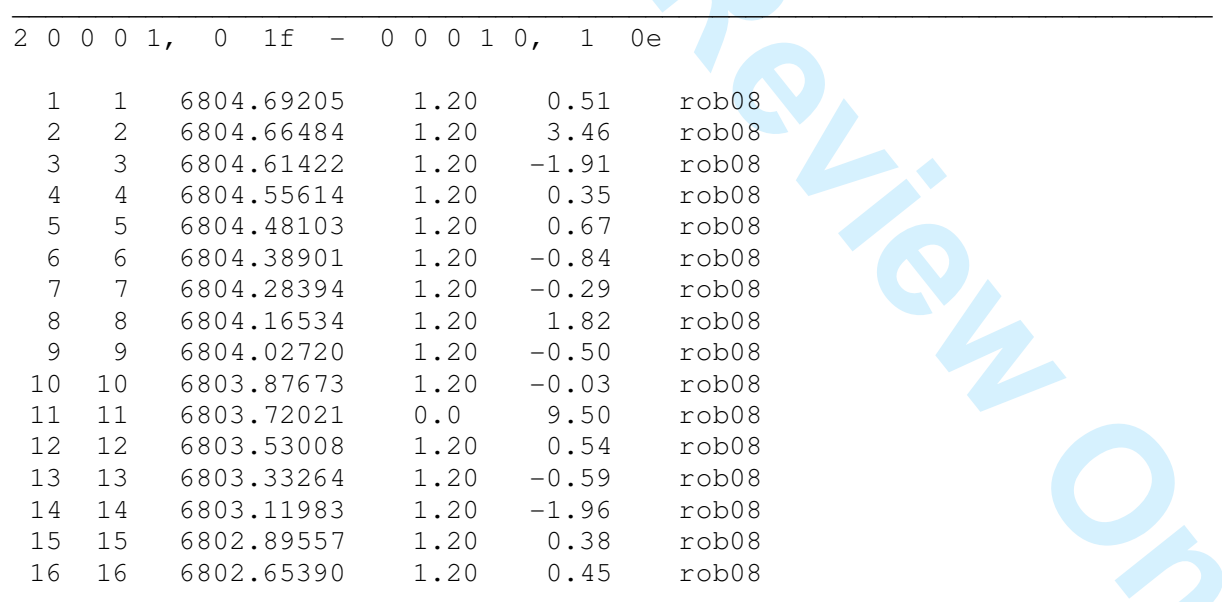

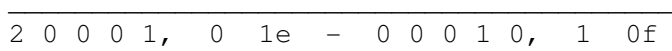

\begin{tabular}{|c|c|c|c|c|}
\hline 1 & 6804.67141 & 1.00 & 0.08 & rob08 \\
\hline 2 & 6804.60156 & 1.00 & 0.83 & rob08 \\
\hline 3 & 6804.49370 & 1.00 & -1.14 & rob08 \\
\hline 4 & 6804.35397 & 1.00 & 0.30 & rob0 8 \\
\hline 5 & 6804.17509 & 1.00 & -2.12 & rob0 8 \\
\hline 6 & 6803.96481 & 1.00 & -0.67 & rob08 \\
\hline 7 & 6803.72078 & 1.00 & 2.30 & rob0 8 \\
\hline 8 & 6803.43633 & 1.00 & 0.10 & rob08 \\
\hline 9 & 6803.11959 & 1.00 & 0.84 & rob08 \\
\hline 10 & 6802.76531 & 1.00 & -0.73 & rob08 \\
\hline 11 & 6802.37915 & 1.00 & 1.02 & rob0 8 \\
\hline 12 & 6801.95485 & 1.00 & -0.17 & robo8 \\
\hline 13 & 6801.49674 & 1.00 & -0.01 & rob0 8 \\
\hline 14 & 6801.00216 & 1.00 & -1.17 & rob08 \\
\hline 15 & 6800.47443 & 1.00 & -0.36 & rob08 \\
\hline 17 & 6799.31211 & 1.00 & -0.34 & rob08 \\
\hline
\end{tabular}




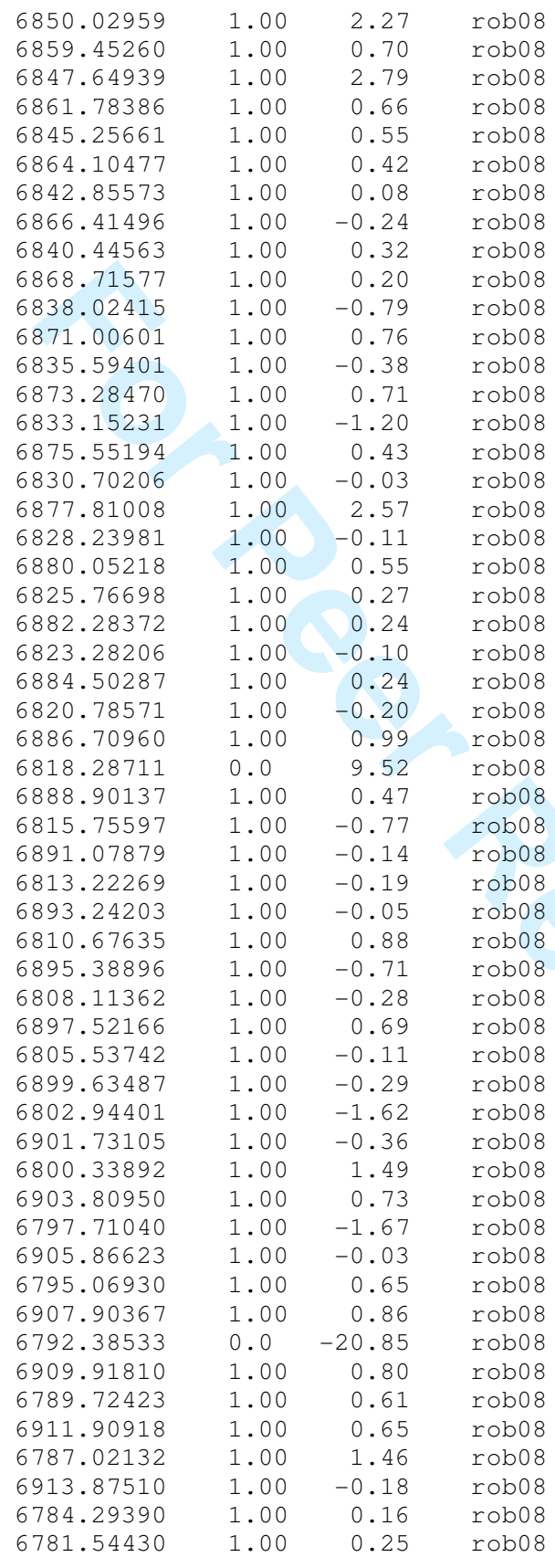

\begin{tabular}{rccccrrrr}
\hline 1 & 1 & 0 & 3, & 0 & $1 e-$ & 0.01 & 0,1 & 0 e \\
& & & & & & \\
2 & 1 & 6850.03431 & 1.60 & -4.52 & rob08 \\
2 & 3 & 6859.40296 & 1.60 & 0.23 & rob08 \\
3 & 2 & 6861.69639 & 1.60 & 1.07 & rob08 \\
3 & 4 & 6845.24409 & 1.60 & 2.69 & rob08 \\
4 & 3 & 6863.96892 & 1.60 & 0.54 & rob08 \\
4 & 5 & 6842.81379 & 1.60 & -0.01 & rob08 \\
5 & 4 & 6866.22036 & 1.60 & -1.39 & rob08 \\
5 & 6 & 6840.36648 & 1.60 & -0.40 & rob08 \\
6 & 5 & 6868.45537 & 1.60 & 0.09 & rob08
\end{tabular}




$\begin{array}{lrrl}6837.90036 & 1.60 & -0.19 & \text { rob08 } \\ 6870.66842 & 1.60 & -0.37 & \text { rob08 } \\ 6835.41545 & 1.60 & 0.75 & \text { rob08 } \\ 6872.86189 & 1.60 & -0.18 & \text { rob08 } \\ 6832.90930 & 1.60 & 0.08 & \text { rob08 } \\ 6875.03529 & 1.60 & 0.40 & \text { rob08 } \\ 6830.38540 & 1.60 & 1.44 & \text { rob08 } \\ 6877.18688 & 1.60 & -0.15 & \text { rob08 } \\ 6827.83825 & 1.60 & -0.50 & \text { rob08 } \\ 6879.31741 & 1.60 & -0.80 & \text { rob08 } \\ 6825.27188 & 1.60 & -1.53 & \text { rob08 } \\ 6881.42781 & 1.60 & -0.35 & \text { rob08 } \\ 6822.68706 & 1.60 & -0.69 & \text { rob08 } \\ 6883.51595 & 1.60 & -0.64 & \text { rob08 } \\ 6820.08055 & 1.60 & -1.00 & \text { rob08 } \\ 6885.58235 & 1.60 & -0.85 & \text { rob08 } \\ 6817.45287 & 1.60 & -1.72 & \text { rob08 } \\ 6887.62760 & 1.60 & -0.08 & \text { rob08 } \\ 6814.80526 & 1.60 & -1.39 & \text { rob08 } \\ 6889.64794 & 1.60 & -1.78 & \text { rob08 } \\ 6812.13593 & 1.60 & -1.55 & \text { rob08 } \\ 6891.64858 & 1.60 & -0.42 & \text { rob08 } \\ 6809.44555 & 1.60 & -1.29 & \text { rob08 } \\ 6893.62338 & 1.60 & -1.79 & \text { rob08 } \\ 6806.73288 & 1.60 & -1.61 & \text { rob08 } \\ 6895.57611 & 1.60 & -1.82 & \text { rob08 } \\ 6803.99732 & 1.60 & -2.84 & \text { rob08 } \\ 6897.50792 & 1.60 & 0.98 & \text { rob08 } \\ 6801.24086 & 1.60 & -2.77 & \text { rob08 } \\ 6899.40994 & 1.60 & -1.95 & \text { rob08 } \\ 6798.46378 & 1.60 & -0.85 & \text { rob08 } \\ 6901.29051 & 1.60 & -1.93 & \text { rob08 } \\ 6795.66193 & 1.60 & -1.02 & \text { rob08 } \\ 6903.14627 & 1.60 & -2.04 & \text { rob08 } \\ 6792.83659 & 1.60 & -1.74 & \text { rob08 } \\ 6904.97740 & 1.60 & -1.78 & \text { rob08 } \\ 6789.98926 & 1.60 & -1.31 & \text { rob08 } \\ 6906.78411 & 1.60 & -0.65 & \text { rob08 } \\ 6787.11771 & 1.60 & -1.73 & \text { rob08 } \\ 6908.55885 & 0.0 & -5.93 & \text { rob08 } \\ 6784.22474 & 1.60 & -0.02 & \text { rob08 } \\ 6910.31435 & 0.0 & -4.62 & \text { rob08 }\end{array}$

6910.31435

$0.0-4.62$

robo 8

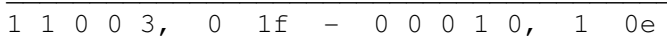

$$
\begin{array}{llllll}
1 & 1 & 6854.76329 & 2.00 & 3.34 & \text { rob08 }
\end{array}
$$

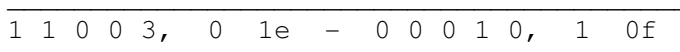$$
\begin{array}{llllll}
1 & 1 & 6854.73341 & 1.40 & 3.80 & \text { rob08 }
\end{array}
$$$$
\begin{array}{llllll}
1 & 1 & 6854.73341 & 1.40 & 3.80 & \text { rob08 } \\
3 & 2 & 6854.67152 & 1.40 & 1.41 & \text { rob08 }
\end{array}
$$$$
\begin{array}{llllll}
3 & 3 & 6854.58106 & 1.40 & 0.26 & \text { rob08 }
\end{array}
$$$$
\begin{array}{llllll}
4 & 4 & 6854.46091 & 1.40 & -0.71 & \text { rob08 }
\end{array}
$$$$
\begin{array}{llllll}
5 & 5 & 6854.31276 & 1.40 & 0.30 & \text { rob08 }
\end{array}
$$$$
\begin{array}{llllll}
6 & 6 & 6854.13259 & 1.40 & -0.62 & \text { rob08 }
\end{array}
$$$$
\begin{array}{llllll}
7 & 7 & 6853.92365 & 1.40 & -0.08 & \text { rob08 }
\end{array}
$$$$
\begin{array}{llllll}
8 & 8 & 6853.68288 & 1.40 & -0.99 & \text { rob08 }
\end{array}
$$

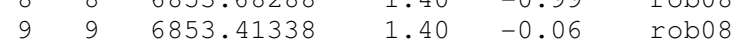$$
\begin{array}{llllll}
10 & 10 & 6853.11175 & 1.40 & -0.50 & \text { rob08 }
\end{array}
$$

\begin{tabular}{rrrrrrllll}
\hline 0 & 0 & 2 & 0, & 2 & $0 e-$ & 0 & 0 & 1,1 & $1 \mathrm{l}$ \\
& & & & & & \\
3 & 4 & 6333.09009 & 2.00 & -1.03 & rob08 \\
4 & 5 & 6330.62667 & 2.00 & -1.86 & rob08 \\
5 & 4 & 6354.08678 & 0.40 & 0.48 & kep96 \\
5 & 6 & 6328.13225 & 0.0 & 2.40 & kep96 \\
6 & 5 & 6356.26651 & 0.40 & -0.93 & kep96 \\
6 & 7 & 6325.59206 & 0.40 & 0.47 & kep96 \\
7 & 6 & 6358.40889 & 0.0 & 1.50 & kep96 \\
7 & 8 & 6323.01269 & 0.40 & 0.56 & kep96 \\
8 & 7 & 6360.50606 & 0.40 & 0.12 & kep96
\end{tabular}




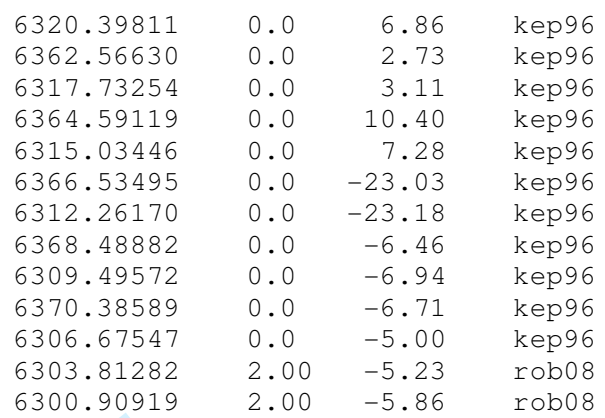

$\begin{array}{rrrrrl}1 & 1 & 6956.90016 & 2.00 & -0.17 & \text { rob08 } \\ 2 & 2 & 6956.85428 & 2.00 & 0.24 & \text { rob08 } \\ 3 & 3 & 6956.78798 & 2.00 & -0.78 & \text { rob08 } \\ 4 & 4 & 6956.70822 & 2.00 & -0.64 & \text { rob08 } \\ 5 & 5 & 6956.61836 & 2.00 & -0.40 & \text { rob08 } \\ 6 & 6 & 6956.52169 & 2.00 & 0.15 & \text { rob08 } \\ 7 & 7 & 6956.41879 & 2.00 & 0.40 & \text { rob08 } \\ 8 & 8 & 6956.31342 & 2.00 & 4.45 & \text { rob08 } \\ 9 & 9 & 6956.19402 & 2.00 & 1.81 & \text { rob08 } \\ 10 & 10 & 6956.06849 & 2.00 & 1.58 & \text { rob08 } \\ 11 & 11 & 6955.93338 & 2.00 & 1.41 & \text { rob08 } \\ 12 & 12 & 6955.78445 & 2.00 & -2.01 & \text { rob08 } \\ 13 & 13 & 6955.63176 & 2.00 & 2.16 & \text { rob08 } \\ 14 & 14 & 6955.46147 & 2.00 & 0.74 & \text { rob08 }\end{array}$




$\begin{array}{rrrlrl}15 & 15 & 6955.28085 & 2.00 & 1.57 & \text { rob08 } \\ 16 & 16 & 6955.08740 & 2.00 & 2.65 & \text { rob08 } \\ 17 & 17 & 6954.87794 & 2.00 & 1.28 & \text { rob08 } \\ 18 & 18 & 6954.65611 & 2.00 & 1.53 & \text { rob08 } \\ 19 & 19 & 6954.42587 & 0.0 & 7.76 & \text { rob08 } \\ 20 & 20 & 6954.17045 & 2.00 & 3.60 & \text { rob08 } \\ 21 & 21 & 6953.90045 & 2.00 & 0.01 & \text { rob08 } \\ 22 & 22 & 6953.61181 & 0.0 & -6.70 & \text { rob08 } \\ 23 & 23 & 6953.31904 & 2.00 & -1.69 & \text { rob08 } \\ 24 & 24 & 6953.00606 & 2.00 & -0.69 & \text { rob08 } \\ 25 & 25 & 6952.67448 & 2.00 & -1.78 & \text { rob08 } \\ 26 & 26 & 6952.32610 & 0.0 & -2.83 & \text { rob08 } \\ 27 & 27 & 6951.96261 & 2.00 & -1.84 & \text { rob08 }\end{array}$

$00220,000-00001, \quad 0 \quad 1 e$

\begin{tabular}{|c|c|c|c|c|c|}
\hline 0 & 1 & 6954.57196 & 1.40 & 0.52 & rob0 8 \\
\hline 1 & 2 & 6952.20428 & 1.40 & 0.27 & rob0 8 \\
\hline 2 & 1 & 6961.58856 & 1.40 & 0.62 & rob0 8 \\
\hline 2 & 3 & 6949.82298 & 1.40 & -0.78 & rob08 \\
\hline 3 & 2 & 6963.90302 & 1.40 & -0.58 & rob0 8 \\
\hline 3 & 4 & 6947.43350 & 1.40 & -0.52 & rob0 8 \\
\hline 4 & 3 & 6966.21321 & 1.40 & -0.71 & rob0 8 \\
\hline 4 & 5 & 6945.03867 & 1.40 & -0.55 & robo 8 \\
\hline 5 & 4 & 6968.52314 & 1.40 & -0.13 & rob08 \\
\hline 5 & 6 & 6942.64347 & 1.40 & -0.32 & rob0 8 \\
\hline 6 & 5 & 6970.83380 & 1.40 & -0.89 & rob0 8 \\
\hline 6 & 7 & 6940.25023 & 1.40 & -0.64 & rob0 8 \\
\hline 7 & 6 & 6973.14888 & 1.40 & -0.44 & rob0 8 \\
\hline 7 & 8 & 6937.86194 & 1.40 & 0.27 & rob0 8 \\
\hline 8 & 7 & 6975.46773 & 1.40 & 0.96 & rob0 8 \\
\hline 8 & 9 & 6935.47717 & 1.40 & 1.30 & rob0 8 \\
\hline 9 & 8 & 6977.78816 & 1.40 & 2.23 & rob0 8 \\
\hline 9 & 10 & 6933.09313 & 1.40 & 0.67 & rob08 \\
\hline 10 & 9 & 6980.10694 & 1.40 & 1.38 & rob0 8 \\
\hline 10 & 11 & 6930.71285 & 1.40 & 2.60 & robo 8 \\
\hline 11 & 10 & 6982.42615 & 1.40 & 1.65 & rob0 8 \\
\hline 11 & 12 & 6928.32979 & 1.40 & 1.61 & rob0 8 \\
\hline 12 & 11 & 6984.73702 & 0.0 & -4.76 & rob0 8 \\
\hline 12 & 13 & 6925.94652 & 1.40 & 1.18 & rob0 8 \\
\hline 13 & 12 & 6987.05974 & 1.40 & 3.18 & rob08 \\
\hline 13 & 14 & 6923.56356 & 1.40 & 2.57 & rob0 8 \\
\hline 14 & 13 & 6989.37066 & 1.40 & 2.52 & rob08 \\
\hline 14 & 15 & 6921.17524 & 1.40 & 0.75 & rob0 8 \\
\hline 15 & 14 & 6991.67462 & 1.40 & -1.28 & rob0 8 \\
\hline 15 & 16 & 6918.78628 & 1.40 & 0.98 & rob0 8 \\
\hline
\end{tabular}

$00220, \quad 200 \quad-\quad 000001, \quad 0 \quad 1 e$

$\begin{array}{rrrlrl}2 & 1 & 6960.97393 & 1.80 & -1.59 & \text { rob08 } \\ 2 & 3 & 6949.21128 & 1.80 & -0.06 & \text { rob08 } \\ 3 & 2 & 6963.27674 & 1.80 & -0.55 & \text { rob08 } \\ 3 & 4 & 6946.79913 & 0.0 & -8.58 & \text { rob08 } \\ 4 & 3 & 6965.55272 & 1.80 & -1.89 & \text { rob08 } \\ 4 & 5 & 6944.37850 & 1.80 & -1.41 & \text { rob08 } \\ 5 & 4 & 6967.80029 & 1.80 & -2.61 & \text { rob08 } \\ 5 & 6 & 6941.92391 & 1.80 & 0.49 & \text { rob08 } \\ 6 & 5 & 6970.01912 & 1.80 & 0.30 & \text { rob08 } \\ 6 & 7 & 6939.43420 & 1.80 & -0.80 & \text { rob08 } \\ 7 & 6 & 6972.20143 & 1.80 & 0.47 & \text { rob08 } \\ 7 & 8 & 6936.91336 & 1.80 & 0.05 & \text { rob08 } \\ 8 & 7 & 6974.35045 & 1.80 & 1.10 & \text { rob08 } \\ 8 & 9 & 6934.35876 & 1.80 & 0.30 & \text { rob08 } \\ 9 & 8 & 6976.46741 & 1.80 & 2.67 & \text { rob08 } \\ 9 & 10 & 6931.77346 & 1.80 & 2.19 & \text { rob08 } \\ 10 & 9 & 6978.55425 & 0.0 & 6.26 & \text { rob08 } \\ 10 & 11 & 6929.15946 & 0.0 & 6.78 & \text { rob08 } \\ 11 & 10 & 6980.57462 & 0.0 & -25.20 & \text { rob08 } \\ 11 & 12 & 6926.48031 & 0.0 & -23.19 & \text { rob08 } \\ 12 & 11 & 6982.61132 & 0.0 & -9.46 & \text { rob08 } \\ 12 & 13 & 6923.81620 & 0.0 & -8.14 & \text { rob08 }\end{array}$




$\begin{array}{llllll}13 & 12 & 6984.60656 & 1.80 & -4.66 & \text { rob08 } \\ 13 & 14 & 6921.11088 & 1.80 & -4.77 & \text { rob08 } \\ 14 & 13 & 6986.56813 & 1.80 & -3.25 & \text { rob08 } \\ 14 & 15 & 6918.37293 & 1.80 & -4.80 & \text { rob08 } \\ 15 & 14 & 6988.49827 & 1.80 & -3.12 & \text { rob08 } \\ 15 & 16 & 6915.60712 & 1.80 & -3.68 & \text { rob08 } \\ 16 & 15 & 6990.39742 & 1.80 & -3.89 & \text { rob08 } \\ 16 & 17 & 6912.81185 & 1.80 & -3.14 & \text { rob08 } \\ 17 & 16 & 6992.26816 & 1.80 & -2.98 & \text { rob08 } \\ 17 & 18 & 6909.98797 & 1.80 & -2.41 & \text { rob08 } \\ 18 & 17 & 6994.10353 & 0.0 & -7.31 & \text { rob08 } \\ 18 & 19 & 6907.13494 & 1.80 & -2.07 & \text { rob08 }\end{array}$

$\begin{array}{lllllllllllllllll}0 & 0 & 2 & 2 & 0, & 2 & 0 \mathrm{f} & - & 0 & 0 & 0 & 0 & 1, & 0 & 1 \mathrm{f}\end{array}$

$\begin{array}{llllll}2 & 1 & 6960.96809 & 1.20 & 0.25 & \text { robo8 }\end{array}$

$\begin{array}{llllll}2 & 3 & 6949.15659 & 1.20 & -0.09 & \text { robo8 }\end{array}$

$\begin{array}{rrrrrr}3 & 2 & 6963.25791 & 1.20 & 0.34 & \text { rob08 } \\ 3 & 4 & 6946.72041 & 1.20 & -1.81 & \text { rob08 }\end{array}$

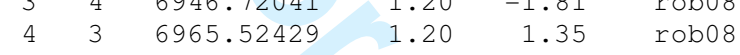

$456944.26289 \quad 1.20 \quad-0.81 \quad$ rob08

$\begin{array}{llllll}5 & 4 & 6967.76384 & 1.20 & 0.00 & \text { rob08 }\end{array}$

$5666941.78035 \quad 1.20 \quad-0.70 \quad$ rob08

$\begin{array}{llllll}6 & 5 & 6969.98007 & 1.20 & -0.06 & \text { rob08 }\end{array}$

$\begin{array}{llllll}6 & 7 & 6939.27484 & 1.20 & 0.60 & \text { rob08 }\end{array}$

$\begin{array}{llllll}7 & 6 & 6972.16863 & 1.20 & -3.03 & \text { rob08 }\end{array}$

$\begin{array}{llllll}7 & 8 & 6936.74051 & 1.20 & -2.68 & \text { rob08 }\end{array}$

$\begin{array}{llllll}8 & 7 & 6974.33697 & 1.20 & -1.28 & \text { rob08 }\end{array}$

$\begin{array}{llllll}8 & 9 & 6934.18681 & 1.20 & -0.99 & \text { rob08 }\end{array}$

$96 \quad 86976.48009 \quad 1.20 \quad 0.38 \quad$ rob08

$\begin{array}{rrrlll}9 & 10 & 6931.60382 & 0.0 & -4.14 & \text { robo8 } \\ 10 & 9 & 6978.59451 & 1.20 & -1.34 & \text { robo8 }\end{array}$

$\begin{array}{llllll}10 & 11 & 6929.00415 & 1.20 & 0.58 & \text { rob08 } \\ 11 & 10 & 6980.68811 & 1.20 & 1.65 & \text { rob08 }\end{array}$

$\begin{array}{llllll}11 & 12 & 6926.37340 & 1.20 & -1.08 & \text { rob08 }\end{array}$

$\begin{array}{llllll}12 & 11 & 6982.75112 & 1.20 & -0.19 & \text { rob08 }\end{array}$

$\begin{array}{llllll}12 & 13 & 6923.72019 & 1.20 & -0.37 & \text { robo8 }\end{array}$

$\begin{array}{llllll}13 & 12 & 6984.78853 & 1.20 & -1.64 & \text { rob08 }\end{array}$

$\begin{array}{llllll}13 & 14 & 6921.04197 & 1.20 & 0.32 & \text { rob08 }\end{array}$

$\begin{array}{llllll}14 & 13 & 6986.80298 & 1.20 & 0.18 & \text { rob08 }\end{array}$

$\begin{array}{llllll}14 & 15 & 6918.33738 & 1.20 & -0.20 & \text { rob08 }\end{array}$

$\begin{array}{llllll}15 & 14 & 6988.78790 & 1.20 & -1.05 & \text { rob08 }\end{array}$

$\begin{array}{llllll}15 & 16 & 6915.60712 & 1.20 & -1.08 & \text { rob08 }\end{array}$

$\begin{array}{llllll}16 & 15 & 6990.74823 & 1.20 & -0.14 & \text { robo8 }\end{array}$

$\begin{array}{llllll}16 & 17 & 6912.85316 & 1.20 & -0.17 & \text { rob08 }\end{array}$

$\begin{array}{llllll}17 & 16 & 6992.67759 & 1.20 & -3.21 & \text { rob08 }\end{array}$

$\begin{array}{llllll}17 & 18 & 6910.07230 & 1.20 & -0.50 & \text { rob08 }\end{array}$

$\begin{array}{llllll}18 & 17 & 6994.58504 & 1.20 & -0.94 & \text { robo8 }\end{array}$

$\begin{array}{llllll}18 & 19 & 6907.26514 & 1.20 & -1.28 & \text { rob08 }\end{array}$

$\begin{array}{llllll}19 & 18 & 6996.46211 & 1.20 & -1.55 & \text { rob08 }\end{array}$

$1920 \quad 6904.43423 \quad 1.20 \quad 0.22 \quad$ rob08

$20 \quad 19 \quad 6998.31059 \quad 1.20 \quad-2.98 \quad$ rob08

$\begin{array}{llllll}20 & 21 & 6901.57377 & 1.20 & -1.64 & \text { rob08 }\end{array}$

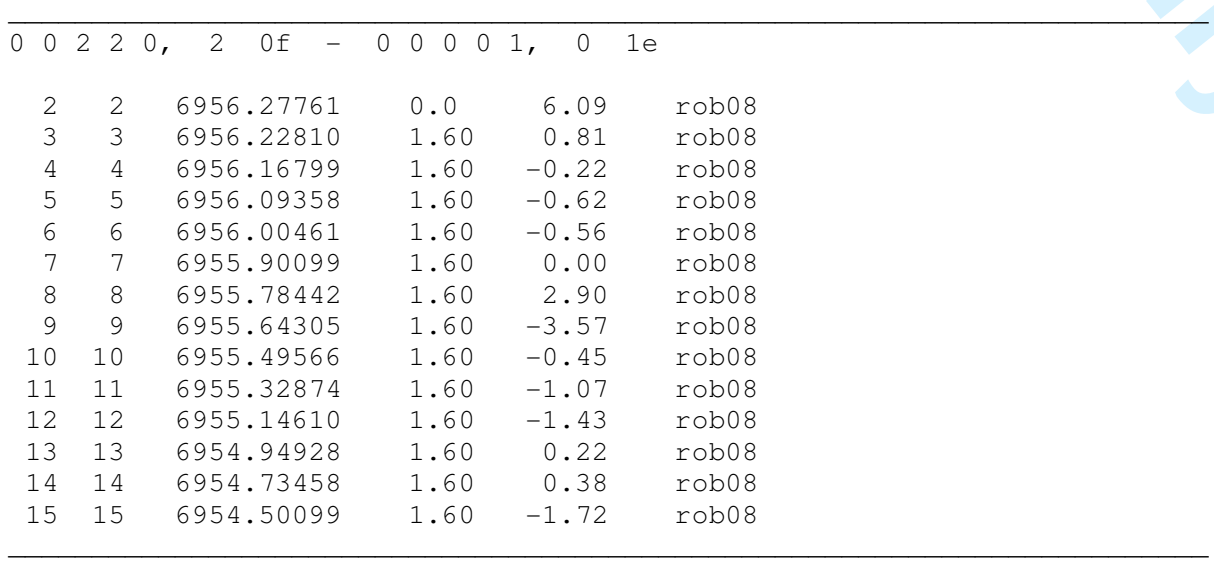


$0 \quad 0211, \quad 1-1 e-00002, \quad 0 \quad 0 e$

$\begin{array}{llllll}0 & 1 & 6353.52974 & 1.00 & -1.16 & \text { rob08 }\end{array}$

$\begin{array}{llllll}1 & 2 & 6351.15515 & 0.0 & 8.68 & \text { rob08 }\end{array}$

$236348.73959 \quad 0.40 \quad-0.47 \quad$ kep96

$\begin{array}{llllll}3 & 4 & 6346.31002 & 0.0 & -1.75 & k e p 96\end{array}$

$4 \quad 3 \quad 6365.11897 \quad 0.40 \quad-1.02 \quad$ kep96

$\begin{array}{llllll}4 & 5 & 6343.86159 & 0.40 & -0.10 & \text { kep96 }\end{array}$

$\begin{array}{llllll}5 & 4 & 6367.37005 & 0.40 & -0.42 & k e p 96\end{array}$

$\begin{array}{llllll}5 & 6 & 6341.39024 & 0.40 & 0.33 & \text { kep96 }\end{array}$

$\begin{array}{llllll}6 & 5 & 6369.59804 & 0.40 & -0.21 \quad k e p 96\end{array}$

$\begin{array}{llllll}6 & 7 & 6338.89592 & 0.40 & -0.61 & \text { kep96 }\end{array}$

$\begin{array}{llllll}7 & 6 & 6371.79767 & 0.0 & -5.56 & \text { kep96 }\end{array}$

$\begin{array}{llllll}7 & 8 & 6336.38238 & 0.40 & 0.75 & \text { kep96 }\end{array}$

$\begin{array}{llllll}8 & 7 & 6373.98524 & 0.40 & -0.07 & \text { kep96 }\end{array}$

$\begin{array}{llllll}8 & 9 & 6333.84567 & 0.40 & 0.36 & \text { kep96 }\end{array}$

$\begin{array}{llllll}9 & 8 & 6376.14648 & 0.0 & 2.08 & k e p 96\end{array}$

$\begin{array}{rrrrrr}9 & 10 & 6331.28955 & 0.0 & 1.88 & \text { kep96 }\end{array}$

$\begin{array}{llllll}10 & 9 & 6378.27658 & 0.0 & -3.85 & k e p 96\end{array}$

$\begin{array}{llllll}10 & 11 & 6328.70501 & 0.0 & -3.83 & k e p 96\end{array}$

$\begin{array}{llllll}11 & 12 & 6326.11068 & 0.0 & 1.74 & \text { kep96 }\end{array}$

$\begin{array}{llllll}12 & 13 & 6323.48957 & 0.0 & 1.43 & k e p 96\end{array}$

$\begin{array}{llllll}13 & 12 & 6384.54722 & 0.0 & -2.76 & \text { kep96 }\end{array}$

$\begin{array}{llllll}13 & 14 & 6320.84747 & 0.40 & 0.79 & k e p 96\end{array}$

$14 \quad 13 \quad 6386.59449 \quad 0.40 \quad 0.62 \quad$ kep96

$\begin{array}{llllll}14 & 15 & 6318.18525 & 0.40 & 0.40 & k e p 96\end{array}$

$\begin{array}{llllll}15 & 16 & 6315.50362 & 1.00 & 0.58 & \text { robo8 }\end{array}$

$\begin{array}{llllll}16 & 17 & 6312.80084 & 1.00 & -0.93 & \text { rob08 }\end{array}$

$\begin{array}{llllll}17 & 18 & 6310.07941 & 0.0 & -2.36 & \text { rob08 }\end{array}$

$\begin{array}{llllll}18 & 19 & 6307.34139 & 0.0 & -2.56 & \text { rob08 }\end{array}$

\begin{tabular}{llllll}
\hline 0 & 1 & 6761.64265 & 2.00 & -2.75 & rob08
\end{tabular}

$\begin{array}{llllll}1 & 0 & 6766.34214 & 2.00 & 3.49 & \end{array}$

$\begin{array}{llllll}1 & 2 & 6759.26516 & 2.00 & 3.01 & \text { rob08 }\end{array}$

$\begin{array}{llllll}2 & 1 & 6768.65105 & 2.00 & 2.12 & \text { rob08 }\end{array}$

$\begin{array}{llllll}2 & 3 & 6756.85594 & 2.00 & 2.39 & \text { robo8 }\end{array}$

$\begin{array}{llllll}3 & 2 & 6770.93638 & 2.00 & 1.96 & \text { robo8 }\end{array}$

$\begin{array}{llllll}3 & 4 & 6754.42106 & 2.00 & 2.73 & \text { rob08 }\end{array}$

$\begin{array}{llllll}4 & 3 & 6773.19586 & 2.00 & 1.72 & \text { rob08 }\end{array}$

$\begin{array}{llllll}4 & 5 & 6751.95630 & 2.00 & 1.43 & \text { rob08 }\end{array}$

$\begin{array}{llllll}5 & 4 & 6775.42827 & 2.00 & 1.62 & \text { rob08 }\end{array}$

$\begin{array}{llllll}5 & 6 & 6749.46201 & 2.00 & 0.82 & \text { robo8 }\end{array}$

$\begin{array}{llllll}6 & 5 & 6777.63170 & 2.00 & 1.54 & \text { rob08 }\end{array}$

$\begin{array}{llllll}6 & 7 & 6746.93762 & 2.00 & 2.46 & \text { robo8 }\end{array}$

$\begin{array}{llllll}7 & 6 & 6779.80337 & 2.00 & 0.84 & \text { rob08 }\end{array}$

$\begin{array}{llllll}7 & 8 & 6744.37589 & 2.00 & 1.27 & \text { rob08 }\end{array}$

$8 \quad 7 \quad 6781.94223$

896741.77882

986784.04456

$9 \quad 10 \quad 6739.14251$

1096786.10879

$10 \quad 11 \quad 6736.46676$

$11 \quad 10 \quad 6788.13326$

$11 \quad 12 \quad 6733.75014$

$\begin{array}{lll}12 & 11 & 6790.11677\end{array}$

$\begin{array}{lll}12 & 13 & 6730.99357\end{array}$

$13 \quad 12 \quad 6792.05611$

$13 \quad 14 \quad 6728.19536$

$\begin{array}{lll}14 & 13 & 6793.95229\end{array}$

$14 \quad 15 \quad 6725.35705$

$15 \quad 14 \quad 6795.80355$

$15 \quad 16 \quad 6722.46891$

$\begin{array}{lll}16 & 15 & 6797.71038\end{array}$

$\begin{array}{lll}16 & 17 & 6719.54054\end{array}$

$17 \quad 16 \quad 6799.53238$

$\begin{array}{lll}18 & 17 & 6801.36335\end{array}$

$19 \quad 18 \quad 6803.33221$

$20 \quad 19 \quad 6805.41651$

$2120 \quad 6807.80831$

$22 \quad 21 \quad 6810.67582$

$2.00 \quad 0.77$

$2.00-1.24$

$2.00-0.05$

$2.00 \quad 0.13$

$2.00-1.05$

$2.00-1.00$

$2.00-2.05$

$2.00-2.71$

$2.00-2.83$

$2.00-3.55$

$2.00-5.58$

$2.00-4.90$

$0.0-8.59$

$2.00-5.01$

$0.0-13.16$

$0.0-13.49$

$0.0 \quad 81.53$

$0.0 \quad-20.57$

$0.0 \quad 135.36$

$0.0 \quad 242.43$

$0.0 \quad 531.94$

$0.0 \quad 981.81$

$0.0 \quad 1784.48$

$0.0 \quad 3108.57$

rob0 8

rob08

robo 8

rob0 8

rob0 8

rob0 8

rob0 8

rob0 8

rob0 8

rob0 8

rob0 8

rob0 8

rob0 8

rob0 8

rob0 8

rob0 8

rob0 8

rob0 8

rob0 8

rob0 8

rob0 8

rob0 8

rob0 8

rob0 8 


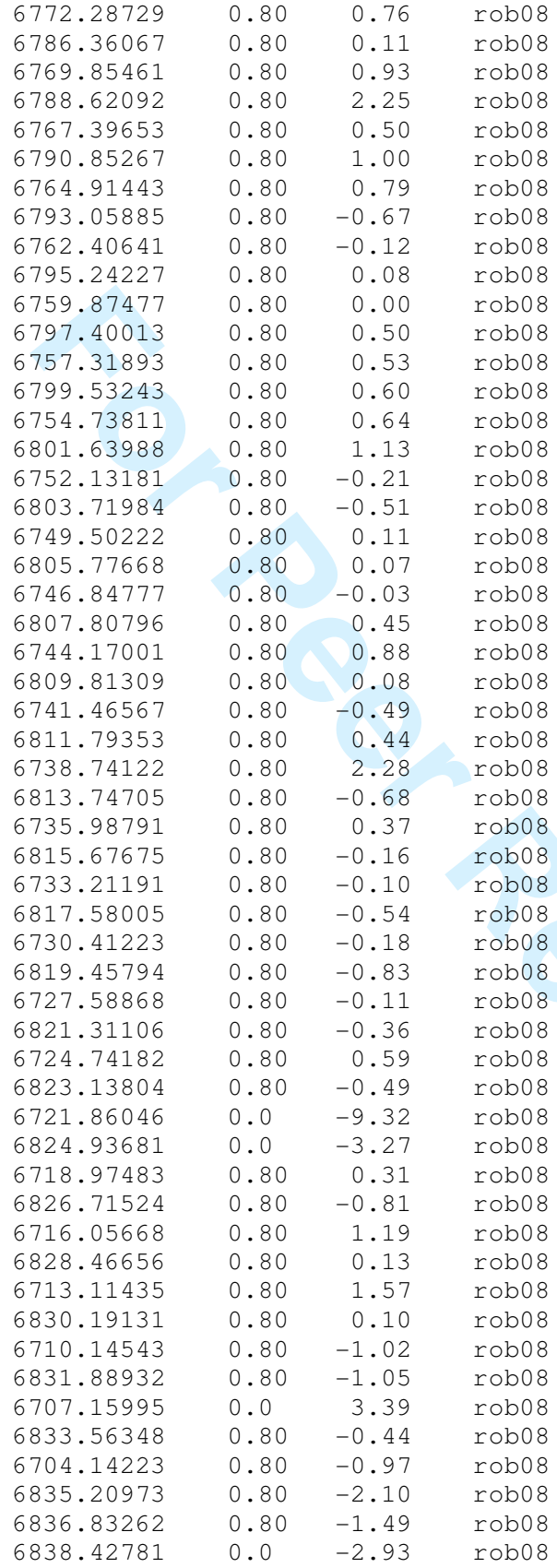




$\begin{array}{rrrlrl}8 & 7 & 6797.45866 & 0.70 & 0.11 & \text { rob08 } \\ 8 & 9 & 6757.45152 & 0.70 & 0.07 & \text { rob08 } \\ 9 & 8 & 6799.62781 & 0.70 & 0.42 & \text { rob08 } \\ 9 & 10 & 6754.93205 & 0.70 & 0.66 & \text { rob08 } \\ 10 & 9 & 6801.78480 & 0.70 & 0.42 & \text { rob08 } \\ 10 & 11 & 6752.40210 & 0.70 & -0.42 & \text { rob08 } \\ 11 & 10 & 6803.93116 & 0.70 & -0.11 & \text { rob08 } \\ 11 & 12 & 6749.86581 & 0.70 & 0.02 & \text { rob08 } \\ 12 & 11 & 6806.06860 & 0.70 & -0.83 & \text { rob08 } \\ 12 & 13 & 6747.32246 & 0.70 & 0.62 & \text { rob08 } \\ 13 & 12 & 6808.19847 & 0.70 & -1.36 & \text { rob08 } \\ 13 & 14 & 6744.77101 & 0.70 & -0.08 & \text { rob08 } \\ 14 & 13 & 6810.32182 & 0.70 & -1.29 & \text { rob08 } \\ 14 & 15 & 6742.21387 & 0.70 & 0.04 & \text { rob08 } \\ 15 & 14 & 6812.43874 & 0.70 & -0.92 & \text { rob08 } \\ 15 & 16 & 6739.65039 & 0.70 & 0.06 & \text { rob08 } \\ 16 & 15 & 6814.54909 & 0.70 & -0.69 & \text { rob08 } \\ 16 & 17 & 6737.08134 & 0.70 & 0.51 & \text { rob08 } \\ 17 & 16 & 6816.65224 & 0.70 & -1.45 & \text { rob08 } \\ 17 & 18 & 6734.50121 & 0.0 & -4.44 & \text { rob08 } \\ 18 & 17 & 6818.75001 & 0.70 & -1.61 & \text { rob08 } \\ 18 & 19 & 6731.92134 & 0.0 & -3.81 & \text { rob08 } \\ 19 & 18 & 6820.84344 & 0.70 & -0.40 & \text { rob08 } \\ 19 & 20 & 6729.33989 & 0.70 & 0.16 & \text { rob08 } \\ 20 & 19 & 6822.92921 & 0.70 & -1.45 & \text { rob08 } \\ 20 & 21 & 6726.74843 & 0.70 & -1.45 & \text { rob08 }\end{array}$

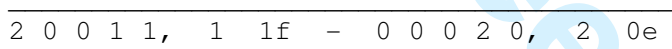

\begin{tabular}{rrrrrl}
2 & 2 & 6779.36149 & 0.80 & 0.09 & rob08 \\
3 & 3 & 6779.28760 & 0.80 & -0.54 & rob08 \\
4 & 4 & 6779.19316 & 0.80 & 0.80 & rob08 \\
5 & 5 & 6779.07640 & 0.80 & 0.79 & rob08 \\
6 & 6 & 6778.93886 & 0.80 & -0.86 & rob08 \\
7 & 7 & 6778.78756 & 0.80 & 0.89 & rob08 \\
8 & 8 & 6778.61953 & 0.80 & 1.14 & rob08 \\
9 & 9 & 6778.43697 & 0.80 & 0.35 & rob08 \\
10 & 10 & 6778.24227 & 0.80 & -0.46 & rob08 \\
11 & 11 & 6778.03669 & 0.80 & -0.96 & rob08 \\
12 & 12 & 6777.82161 & 0.80 & -0.23 & rob08 \\
13 & 13 & 6777.59572 & 0.80 & 0.28 & rob08 \\
\hline
\end{tabular}

$20011, \quad 1 \quad 1 e-000020, \quad 20 f$

$\begin{array}{llllll}2 & 2 & 6779.36149 & 0.70 & 0.46 & \text { rob08 }\end{array}$

$\begin{array}{llllll}3 & 3 & 6779.28760 & 0.70 & 1.28 & \text { rob08 }\end{array}$

$\begin{array}{llllll}4 & 4 & 6779.18838 & 0.70 & 1.47 & \text { robo8 }\end{array}$

$\begin{array}{llllll}5 & 5 & 6779.06362 & 0.70 & 0.62 & \text { rob08 }\end{array}$

$\begin{array}{llllll}6 & 6 & 6778.91516 & 0.70 & 0.37 & \text { rob08 }\end{array}$

$\begin{array}{llllll}7 & 7 & 6778.74234 & 0.70 & -0.23 & \text { rob08 }\end{array}$

$\begin{array}{rrrrrr}8 & 8 & 6778.54698 & 0.70 & 0.32 & \text { rob08 }\end{array}$

$\begin{array}{llllll}9 & 9 & 6778.32728 & 0.70 & -0.12 & \text { rob08 }\end{array}$

$\begin{array}{llllll}10 & 10 & 6778.08607 & 0.70 & 0.85 & \text { rob08 }\end{array}$

$\begin{array}{llllll}11 & 11 & 6777.81994 & 0.70 & -0.62 & \text { rob08 }\end{array}$

$\begin{array}{llllll}12 & 12 & 6777.53284 & 0.70 & -1.06 & \text { rob08 }\end{array}$

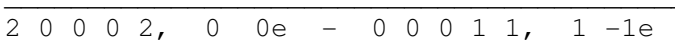

$\begin{array}{rrrrrr}0 & 1 & 6783.92912 & 1.80 & 0.15 & \text { rob08 } \\ 1 & 0 & 6788.62164 & 1.80 & -4.07 & \text { rob08 } \\ 1 & 2 & 6781.54237 & 1.80 & -0.60 & \text { rob08 } \\ 2 & 1 & 6790.93597 & 1.80 & -0.31 & \text { rob08 } \\ 2 & 3 & 6779.13141 & 1.80 & -0.57 & \text { rob08 } \\ 3 & 2 & 6793.22184 & 1.80 & 0.36 & \text { rob08 } \\ 3 & 4 & 6776.69697 & 1.80 & 0.93 & \text { rob08 } \\ 4 & 3 & 6795.48199 & 1.80 & 0.82 & \text { rob08 } \\ 4 & 5 & 6774.23523 & 1.80 & 0.07 & \text { rob08 } \\ 5 & 4 & 6797.71034 & 1.80 & -4.86 & \text { rob08 } \\ 5 & 6 & 6771.74826 & 1.80 & -1.10 & \text { rob08 } \\ 6 & 5 & 6799.92595 & 1.80 & 2.58 & \text { rob08 } \\ 6 & 7 & 6769.24062 & 1.80 & 2.01 & \text { rob08 }\end{array}$




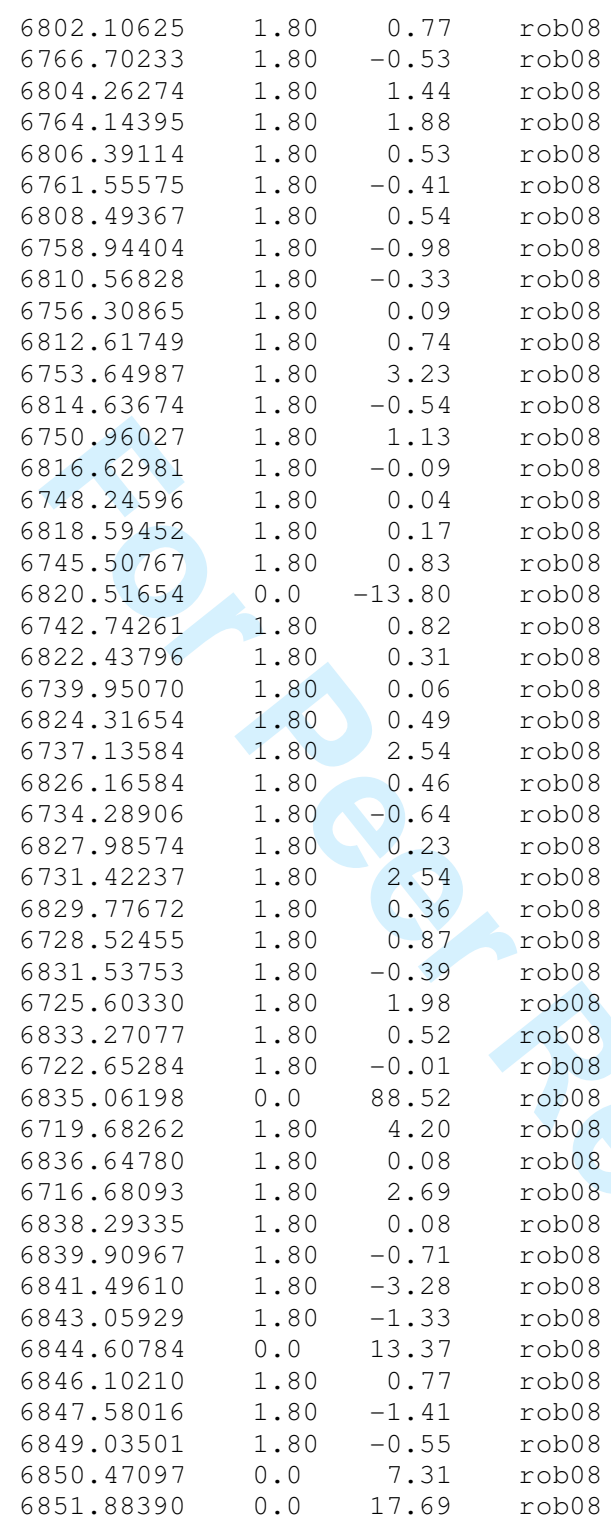




\begin{tabular}{|c|c|c|c|c|c|}
\hline 12 & 13 & 6748.35878 & 1.80 & 0.29 & rob08 \\
\hline 13 & 12 & 6809.35329 & 1.80 & -4.50 & rob0 8 \\
\hline 13 & 14 & 6745.68878 & 0.0 & 9.71 & rob0 8 \\
\hline 14 & 13 & 6811.36430 & 1.80 & $-1 \cdot 32$ & rob08 \\
\hline 14 & 15 & 6742.97403 & 1.80 & -1.29 & rob0 8 \\
\hline 15 & 14 & 6813.34692 & 1.80 & -1.09 & rob08 \\
\hline 15 & 16 & 6740.24689 & 1.80 & -0.38 & rob0 8 \\
\hline 16 & 15 & 6815.30464 & 1.80 & -0.27 & rob08 \\
\hline 16 & 17 & 6737.49525 & 1.80 & 0.30 & robo 8 \\
\hline 17 & 16 & 6817.23524 & 1.80 & -1.03 & robo 8 \\
\hline 17 & 18 & 6734.71931 & 1.80 & 0.92 & rob0 8 \\
\hline 18 & 17 & 6819.14090 & 1.80 & -1.14 & rob0 8 \\
\hline 18 & 19 & 6731.92111 & 1.80 & 3.48 & rob0 8 \\
\hline 19 & 18 & 6821.02198 & 1.80 & -0.21 & rob0 8 \\
\hline 19 & 20 & 6729.09616 & 1.80 & 3.45 & rob0 8 \\
\hline 20 & 19 & 6822.87609 & 1.80 & -0.59 & rob0 8 \\
\hline 20 & 21 & 6726.24728 & 1.80 & 3.63 & rob0 8 \\
\hline 21 & 20 & 6824.70468 & 1.80 & -0.76 & rob0 8 \\
\hline 21 & 22 & 6723.37051 & 1.80 & 0.02 & rob0 8 \\
\hline 22 & 23 & 6720.47305 & 1.80 & -0.22 & rob0 8 \\
\hline 22 & 21 & 6826.50779 & 1.80 & -0.65 & robo 8 \\
\hline 23 & 22 & 6828.28444 & 1.80 & -1.20 & rob0 8 \\
\hline 24 & 23 & 6830.03588 & 1.80 & -1.11 & rob0 8 \\
\hline 25 & 24 & 6831.76050 & 1.80 & -1.94 & robo 8 \\
\hline 26 & 25 & 6833.46005 & 1.80 & -1.90 & robo 8 \\
\hline 27 & 26 & 6835.13347 & 1.80 & -1.99 & rob0 8 \\
\hline 28 & 27 & 6836.78171 & 1.80 & -1.23 & rob0 8 \\
\hline 29 & 28 & 6838.39810 & 0.0 & -6.24 & rob0 8 \\
\hline 30 & 29 & 6839.99808 & 1.80 & -1.53 & rob08 \\
\hline
\end{tabular}

$\begin{array}{lllllllllllllllll}2 & 0 & 0 & 0 & 2, & 0 & 2 e & - & 0 & 0 & 0 & 1 & 1, & 1 & 1 \mathrm{e}\end{array}$

$\begin{array}{rrrlrl}2 & 3 & 6773.80564 & 1.80 & -0.25 & \text { rob08 } \\ 3 & 2 & 6787.88933 & 1.80 & -0.09 & \text { rob08 } \\ 3 & 4 & 6771.37157 & 1.80 & -0.14 & \text { rob08 } \\ 4 & 3 & 6790.15470 & 1.80 & 4.84 & \text { rob08 } \\ 4 & 5 & 6768.91313 & 1.80 & 0.35 & \text { rob08 } \\ 5 & 4 & 6792.38519 & 1.80 & -0.45 & \text { rob08 } \\ 5 & 6 & 6766.42952 & 1.80 & 0.33 & \text { rob08 } \\ 6 & 5 & 6794.59586 & 1.80 & -1.03 & \text { rob08 } \\ 6 & 7 & 6763.92010 & 1.80 & -0.94 & \text { rob08 } \\ 7 & 6 & 6796.78241 & 1.80 & -1.30 & \text { rob08 } \\ 7 & 8 & 6761.40302 & 0.0 & 14.57 & \text { rob08 } \\ 8 & 7 & 6798.94592 & 1.80 & -0.35 & \text { rob08 } \\ 8 & 9 & 6758.83128 & 1.80 & -0.30 & \text { rob08 } \\ 9 & 8 & 6801.08397 & 1.80 & -0.74 & \text { rob08 } \\ 9 & 10 & 6756.25026 & 1.80 & -0.32 & \text { rob08 } \\ 10 & 9 & 6803.19949 & 1.80 & 0.25 & \text { rob08 } \\ 10 & 11 & 6753.64956 & 1.80 & 3.93 & \text { rob08 } \\ 11 & 10 & 6805.28929 & 1.80 & -0.74 & \text { rob08 } \\ 11 & 12 & 6751.01633 & 1.80 & -0.60 & \text { rob08 } \\ 12 & 11 & 6807.35638 & 1.80 & -0.92 & \text { rob08 } \\ 12 & 13 & 6748.35916 & 0.0 & -5.53 & \text { rob08 } \\ 13 & 12 & 6809.40064 & 1.80 & -0.62 & \text { rob08 } \\ 13 & 14 & 6745.68878 & 1.80 & -0.34 & \text { rob08 } \\ 14 & 13 & 6811.42159 & 1.80 & -0.54 & \text { rob08 } \\ 14 & 15 & 6742.98972 & 1.80 & -0.75 & \text { rob08 } \\ 15 & 14 & 6813.42039 & 1.80 & 0.29 & \text { rob08 } \\ 15 & 16 & 6740.26932 & 1.80 & 0.38 & \text { rob08 } \\ 16 & 15 & 6815.39427 & 1.80 & -1.12 & \text { rob08 } \\ 16 & 17 & 6737.52529 & 1.80 & 0.53 & \text { rob08 } \\ 17 & 16 & 6817.34819 & 1.80 & 0.03 & \text { rob08 } \\ 17 & 18 & 6734.75775 & 1.80 & -0.40 & \text { rob08 } \\ 18 & 17 & 6819.27930 & 1.80 & 0.74 & \text { rob08 } \\ 18 & 19 & 6731.96972 & 1.80 & 0.44 & \text { rob08 } \\ 19 & 18 & 6821.18675 & 1.80 & 0.05 & \text { rob08 } \\ 19 & 20 & 6729.15845 & 1.80 & 0.14 & \text { rob08 } \\ 20 & 19 & 6823.07168 & 1.80 & -0.97 & \text { rob08 } \\ 20 & 21 & 6726.32457 & 1.80 & -0.81 & \text { rob08 } \\ 21 & 22 & 6723.48708 & 0.0 & 16.50 & \text { rob08 } \\ 21 & 20 & 6824.93540 & 1.80 & -1.00 & \text { rob08 }\end{array}$




$\begin{array}{rrrlrr}22 & 23 & 6720.58368 & 0.0 & -10.26 & \text { rob08 } \\ 22 & 21 & 6826.78880 & 0.0 & 10.88 & \text { rob08 } \\ 23 & 24 & 6717.68974 & 0.0 & -5.73 & \text { rob08 } \\ 23 & 22 & 6828.59475 & 1.80 & -2.34 & \text { rob08 } \\ 24 & 23 & 6830.36549 & 0.0 & -28.26 & \text { rob08 } \\ 25 & 24 & 6832.16737 & 1.80 & -0.30 & \text { rob08 } \\ 26 & 25 & 6833.95034 & 0.0 & 31.77 & \text { rob08 } \\ 27 & 26 & 6835.64594 & 1.80 & -0.17 & \text { rob08 } \\ 28 & 27 & 6837.32929 & 0.0 & -20.63 & \text { rob08 } \\ 29 & 28 & 6838.98393 & 0.0 & -45.68 & \text { rob08 } \\ 30 & 29 & 6840.60469 & 0.0 & -80.07 & \text { rob08 } \\ 31 & 30 & 6842.20659 & 0.0 & -108.35 & \text { rob08 }\end{array}$

\begin{tabular}{|c|c|c|c|c|c|}
\hline \multicolumn{6}{|c|}{00} \\
\hline 2 & 2 & 6780.88370 & 1.00 & -1.25 & rob0 8 \\
\hline 3 & 3 & 6780.81060 & 1.00 & -0.01 & rob0 8 \\
\hline 4 & 4 & 6780.70978 & 1.00 & -1.99 & rob0 8 \\
\hline 5 & 5 & 6780.58761 & 1.00 & -1.08 & rob0 8 \\
\hline 6 & 6 & 6780.43546 & 0.0 & -6.21 & rob0 8 \\
\hline 7 & 7 & 6780.27182 & 1.00 & 0.73 & rob0 8 \\
\hline 9 & 9 & 6779.86101 & 1.00 & 0.00 & rob0 8 \\
\hline 10 & 10 & 6779.62860 & 0.0 & 6.04 & rob0 8 \\
\hline
\end{tabular}

\begin{tabular}{|c|c|c|c|c|c|c|}
\hline 01 & 10 & 03, & $1 f$ & $\begin{array}{lll}0 & 0 & 0\end{array}$ & 01, & $1 \mathrm{f}$ \\
\hline 1 & 2 & 2 & 6667.49660 & 1.80 & 0.18 & rob08 \\
\hline 2 & 1 & 1 & 6676.92300 & 1.80 & -0.55 & rob08 \\
\hline 3 & 2 & 2 & 6679.25356 & 1.80 & 0.69 & rob08 \\
\hline 4 & 3 & 3 & 6681.57382 & 1.80 & 2.73 & rob08 \\
\hline 5 & 4 & 4 & 6683.87890 & 1.80 & 0.77 & rob0 8 \\
\hline 6 & 5 & 5 & 6686.17581 & 1.80 & 1.91 & rob08 \\
\hline 7 & 6 & 6 & 6688.45899 & 1.80 & 0.72 & rob0 8 \\
\hline 8 & 7 & 7 & 6690.73297 & 1.80 & 1.88 & rob08 \\
\hline 9 & 8 & 8 & 6692.99245 & 1.80 & 0.25 & rob08 \\
\hline 10 & 9 & 9 & 6695.24274 & 1.80 & 1.40 & rob0 8 \\
\hline
\end{tabular}




$\begin{array}{llrl}6697.47888 & 1.80 & 0.62 & \text { rob08 } \\ 6699.70240 & 1.80 & -0.20 & \text { rob08 } \\ 6701.91533 & 1.80 & 1.37 & \text { rob08 } \\ 6704.11214 & 1.80 & 0.31 & \text { rob08 } \\ 6706.29489 & 1.80 & -0.74 & \text { rob08 } \\ 6708.46364 & 1.80 & -1.03 & \text { rob08 } \\ 6710.61678 & 1.80 & -1.38 & \text { rob08 } \\ 6712.75410 & 1.80 & -1.06 & \text { rob08 } \\ 6714.87184 & 1.80 & -2.81 & \text { rob08 } \\ 6716.97344 & 1.80 & -2.02 & \text { rob08 } \\ 6719.05353 & 1.80 & -2.80 & \text { rob08 } \\ 6721.11287 & 1.80 & -3.02 & \text { rob08 } \\ 6723.14936 & 1.80 & -3.33 & \text { rob08 } \\ 6725.16151 & 1.80 & -3.74 & \text { rob08 } \\ 6727.12337 & 0.0 & -28.71 & \text { rob08 } \\ 6729.09609 & 0.0 & -15.64 & \text { rob08 } \\ 6731.03277 & 0.0 & -10.06 & \text { rob08 } \\ 6732.90375 & 0.0 & -40.38 & \text { rob08 }\end{array}$

$\begin{array}{lll}11 & 10 & 6697.47888\end{array}$

$3 \quad 12 \quad 6701.91533$

14136704.11214

$1514 \quad 6706.29489$

$16 \quad 15 \quad 6708.46364$

$\begin{array}{lll}7 & 16 & 6710.61678\end{array}$

$\begin{array}{lll}18 & 17 & 6712.75410\end{array}$

$9 \quad 18 \quad 6714.87184$

$\begin{array}{lll}19 & 6716.97344\end{array}$

$120 \quad 6719.05353$

$\begin{array}{lll}22 & 21 & 6721.11287\end{array}$

$322 \quad 6723.14936$

$4 \quad 23 \quad 6725.16151$

$\begin{array}{lll}5 & 24 & 6727.12337\end{array}$

$\begin{array}{lll}6 & 25 & 6729.09609\end{array}$

$\begin{array}{lll}27 & 26 & 6731.03277\end{array}$

$28 \quad 27$

6732.90375

$0.0 \quad-40.38$

rob08
rob08
rob08
rob0 8
rob08
rob08
rob0 8
rob08
rob08
rob0 8
rob08
rob08
rob0 8
rob08
rob08
rob0 8
rob08
rob08

9

20

21

24

5

26

27

32

33

34

35

36

39

40

41

42

44

45

46

47

48

49

50

52

53

54

55

56 


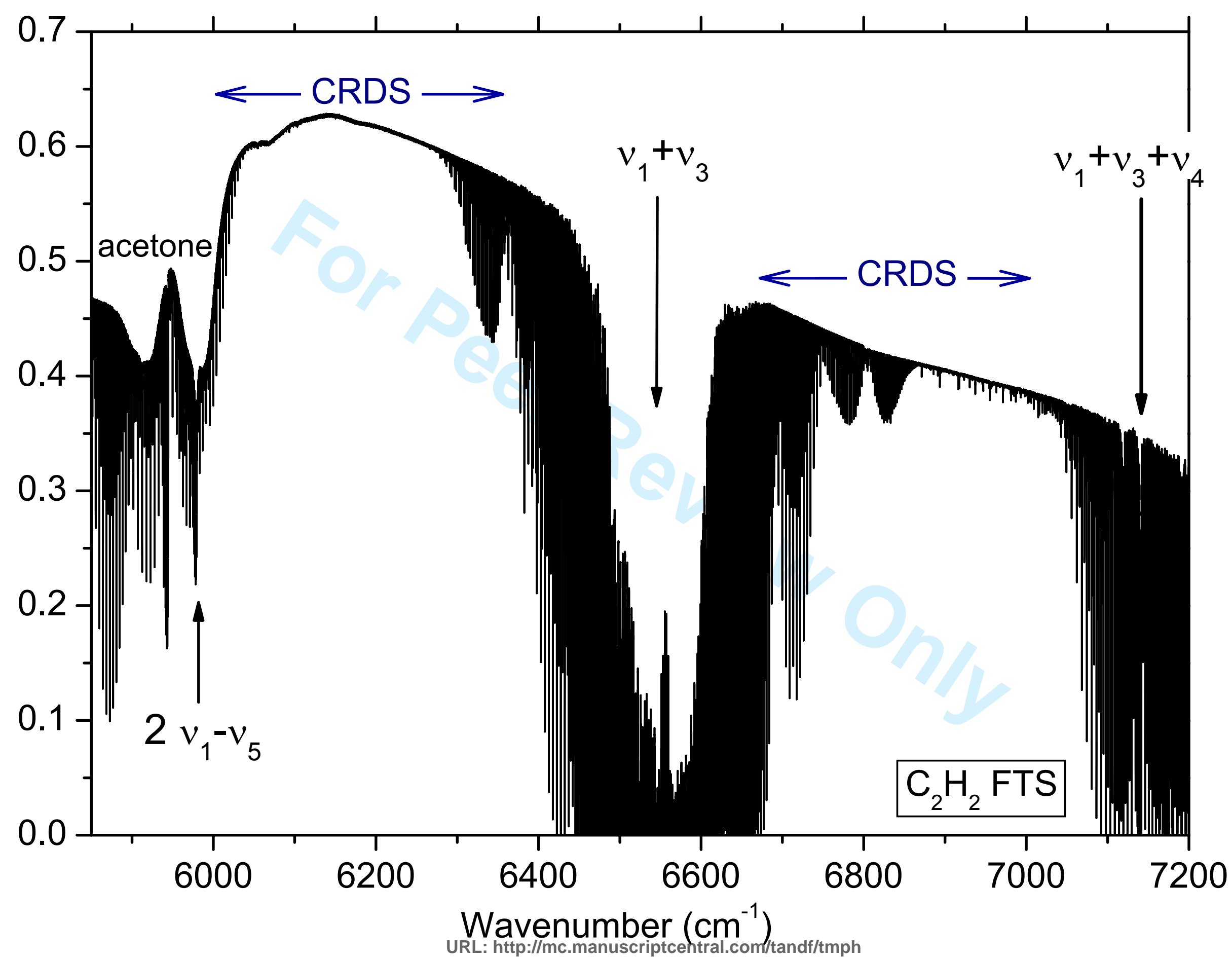

Fig. 1 


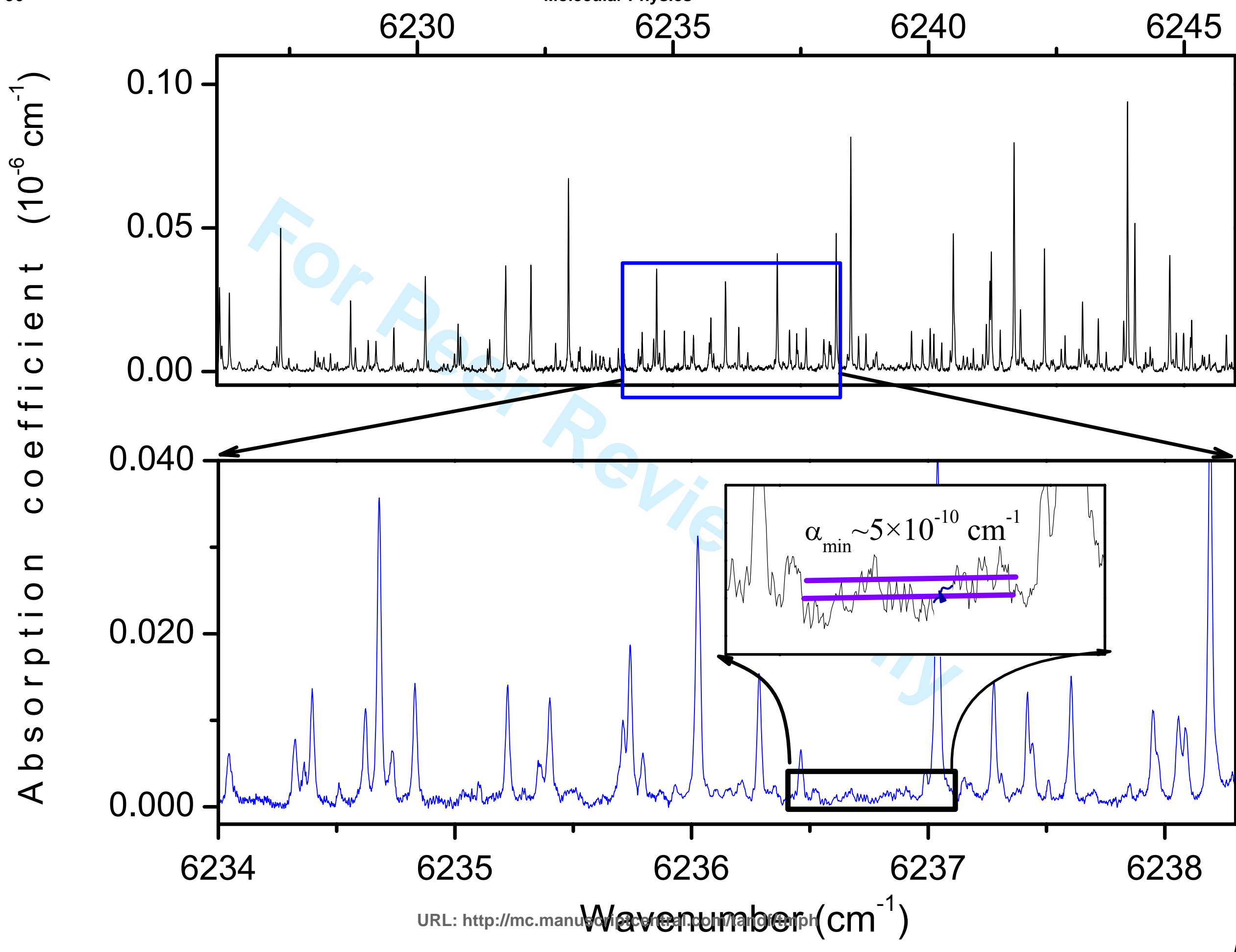

Fig. 2 


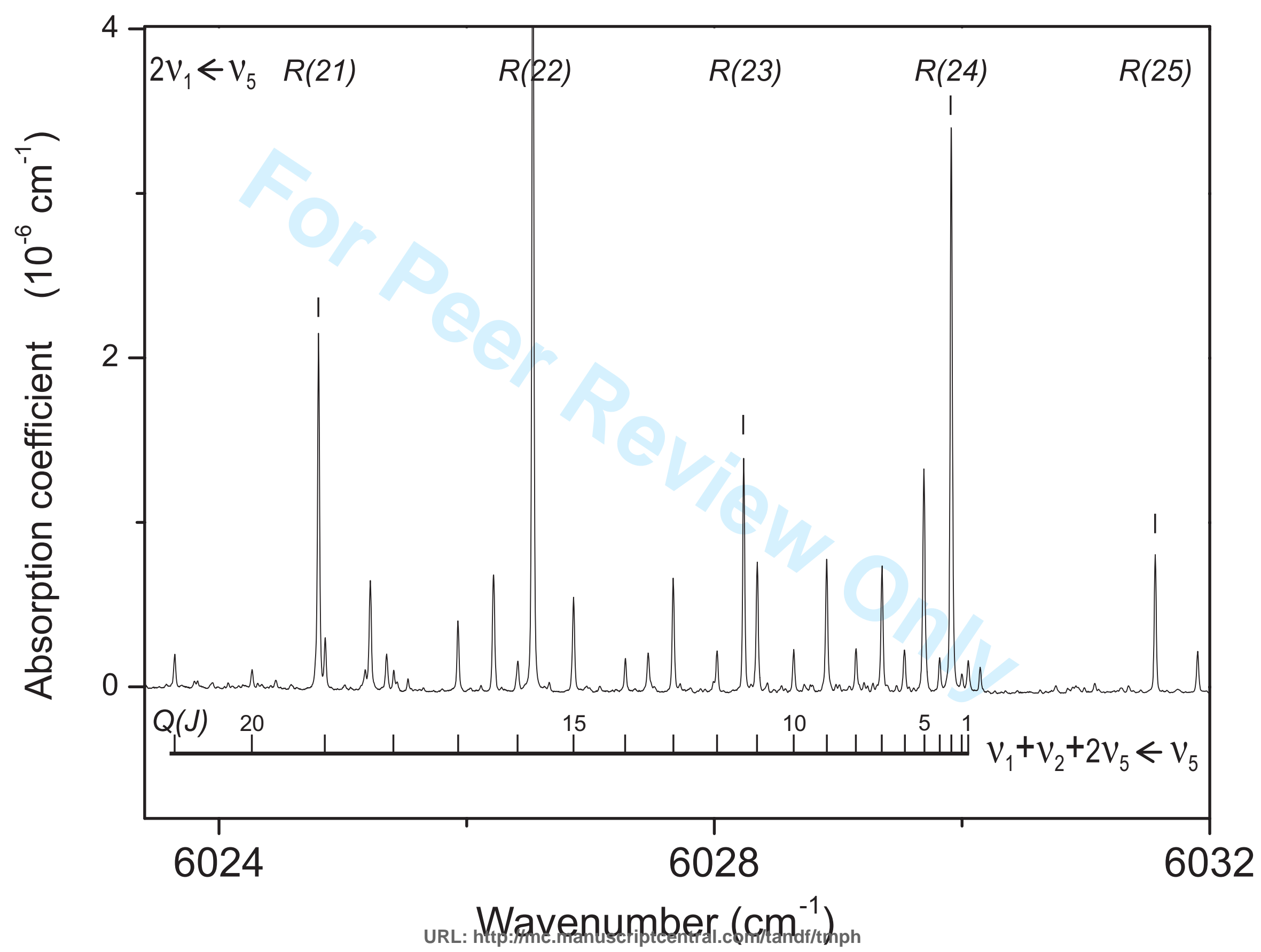




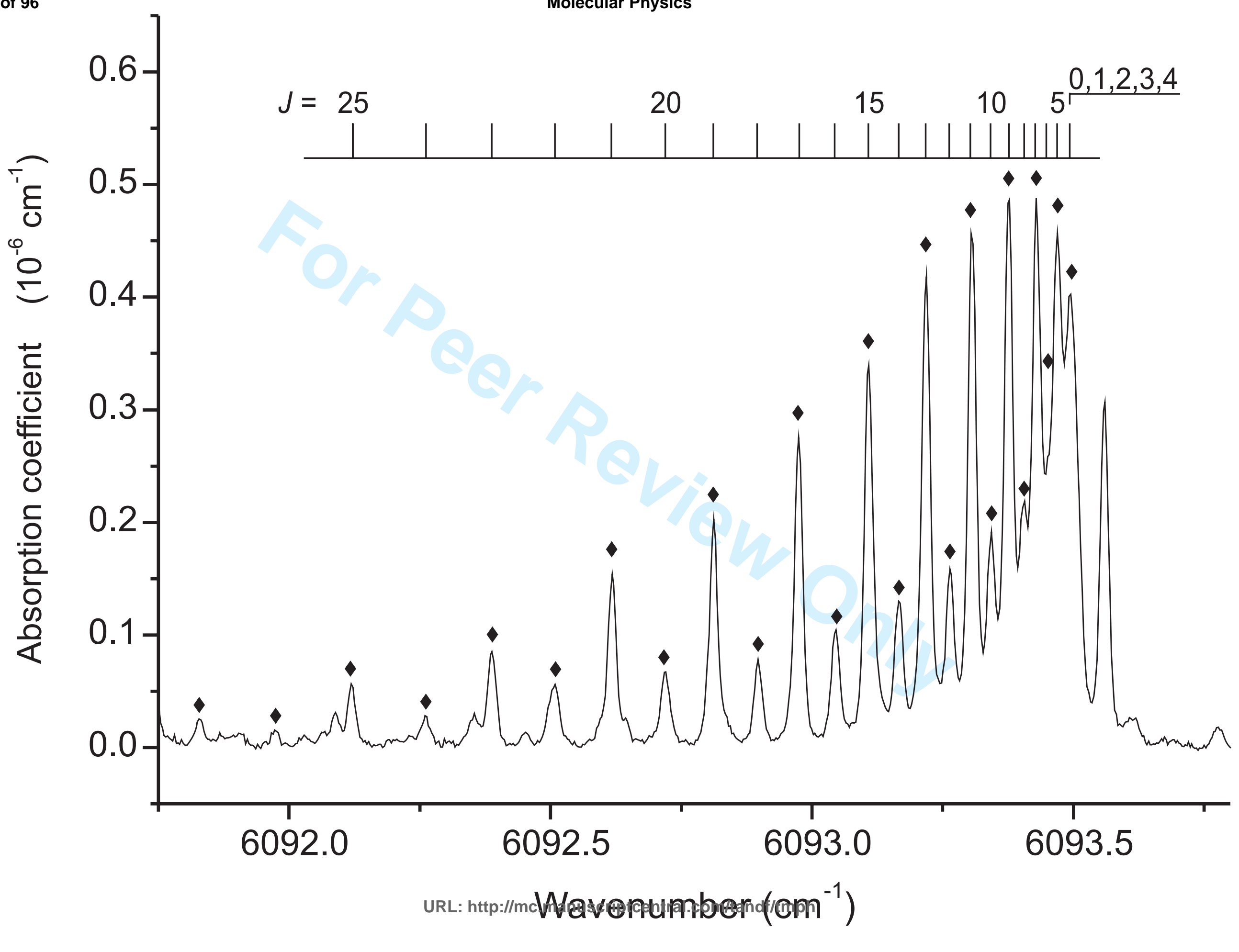




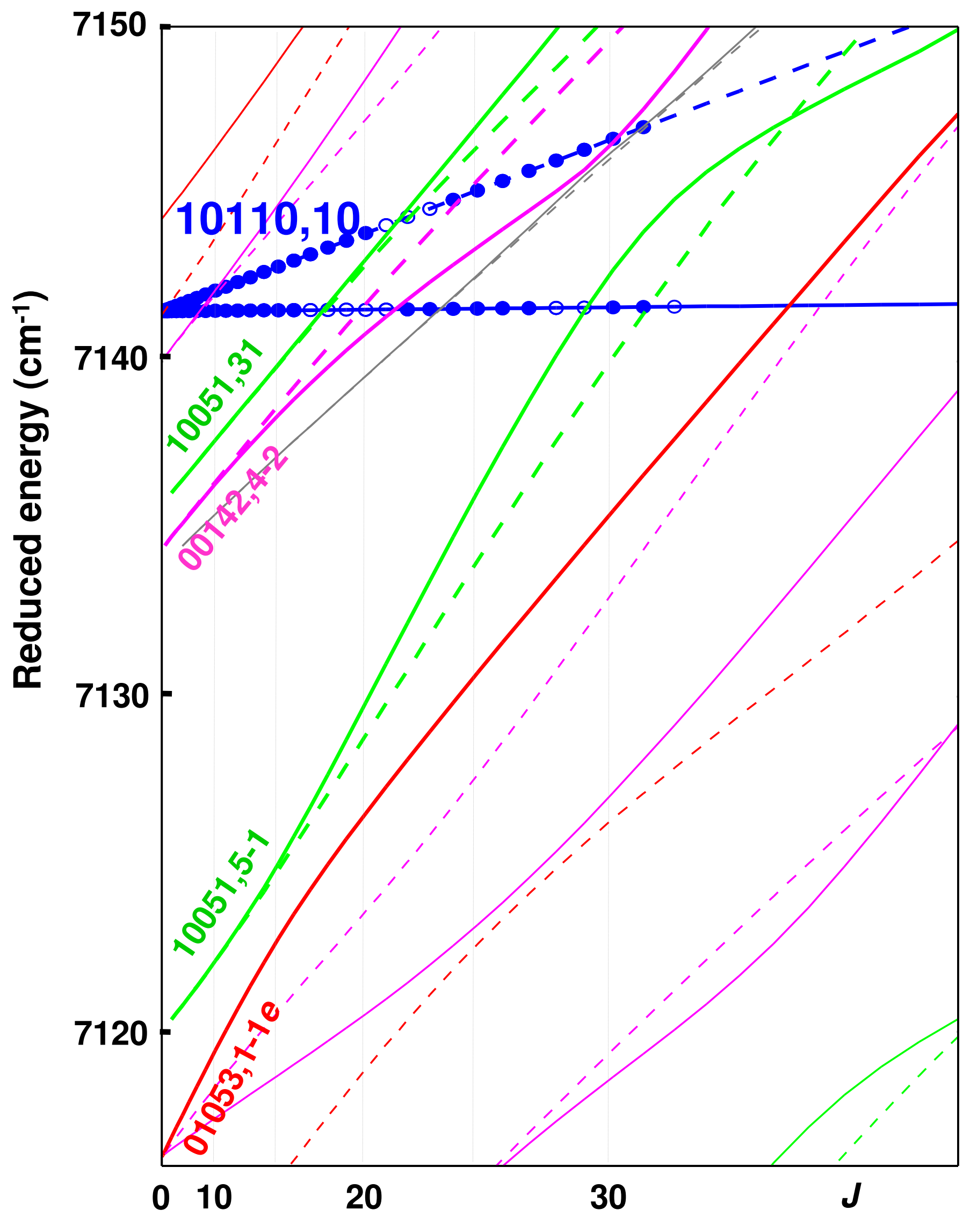

URL: http://mc.manuscriptcentral.com/tandf/tmph 